\title{
O Modelo dos Vórtices em Hidrodinâmica
}

Violeta Nydia Vivanco Orellana

DISSERTAÇÃO APRESENTADA

$\mathrm{AO}$

INSTITUTO DE MATEMÁTICA E ESTATÍSTICA

DA UNIVERSIDADE DE SÃO PAULO

PARA A OBTENÇÃO DO GRAU DE MESTRE

EM

MATEMÁTICA APLICADA

Área de Concentração: Matemática Aplicada

Orientadora: Prof. Dr. Helena Maria Ávila de Castro.

Este trabalho foi financiado pela CAPES

-São Paulo, Junho de 2001- 


\section{Modelo dos Vórtices em Hidrodinâmica}

Este exemplar corresponde à redação final da dissertação devidamente corrigida e defendida por Violeta Nydia Vivanco Orellana e aprovada pela comissão julgadora.

São Paulo, 21 de junho de 2001.

Banca examinadora:

- Prof. Dra. Helena Maria Ávila de Castro (Presidente) - IME - USP

- Prof. Dr. Clodoaldo Grotta Ragazzo - IME - USP

- Prof. Dr. Oscar Brito Augusto -EP- USP 
À Bernardo e a meus quatro filhos:

Leonardo, Dario,

Natalia e Valeria. 


\section{Agradecimentos}

Agradeço a Bernardo por ter-me ensinado que para alcançar um objetivo, antes de tudo, é necessário perseverança.

Aos meus filhos, pela a força que me transmitem e pelo desejo de ensinar-lhes que sendo perseverantes é possível conseguir o que se deseja.

A meu colega Rommel Bustinza por ter me ajudado a compilar, em mais de uma oportunidade, este trabalho.

De maneira muito especial agradaço a minha professora Helena, por haver me orientado com seriedade e eficiência. 


\section{Resumo}

O modelo dos vórtices é definido supondo que o rotacional do campo de velocidade de um fluido ideal e incompressível no plano seja nulo, exceto em um conjunto discreto de pontos chamados vórtices. Como o campo de velocidade é singular nos vórtices, é feita uma hipótese regularizadora para obter as equações que determinam seu movimento: assumindo que cada vórtice não se movimenta pela ação de seu campo, mas sim pela ação do campo gerado pelos outros vórtices, obtém-se as equações de Helmholtz-Kirchhoff.

As equações de Helmholtz-Kirchhoff são extendidas ao caso em que a cada vórtice está associada uma massa, a qual pode ser interpretada como a massa de uma impureza do fluido. Obtém-se assim o modelo dos vórtices com massa.

O modelo misto, isto é, o modelo no qual alguns dos vórtices tem massa e outros não, é obtido a partir do modelo dos vórtices com massa, restrito a uma subvariedade conveniente de seu espaço de fase, mediante o formalismo de Dirac para sistemas Hamiltonianos com vínculos.

Serão apresentados, além disso, diversos sistemas de vórtices (algums com e outros sem massa), mostrando casos integráveis e não integráveis, dependendo do número de vórtices e dos valores dos parâmetros. A não integrabilidade é obtida pelo método de Melnikov ou por meio da aplicação do teorema de Lerman.

Palavras chaves: dinâmica de vórtices, sistemas Hamiltonianos, integrabilidade, variedades de Poisson. 


\begin{abstract}
The vortex model is defined by supossing that the curl of velocity field of an ideal and incompressible two-dimensional fluid vanishes, except at a discrete set of points called vortices. Since the velocity field is singular at the vortices, it's necessary to introduce a regularity hypothesis to get the equation that stablishes its motion: we assume that each vortex is not affected by the action of its oun field, but by the action of the field generated by the other vortices. In this way, one obtains the Helmholtz-Kirchhoff equations.

The Helmholtz-Kirchhoff equations are extended to the case where each vortex has an associated mass, which can be interpreted as the mass of an inpurity of the fluid: this is the mass vortex model.

The mixed model, that is, the model where some of the vortices have a mass, while others do not, is obtained from the mass vortex model restricted to suitable submanifold of its phase space, as suggested by Dirac's formalism for restricted Hamiltonians systems.

Moreover, several vortex systems (some with and other without mass) will be presented, showing integrable and non integrable cases, depending on the number of vortices and on the parameters values. Non integrability is proved by Melnikov's method or by appliying Lerman's theorem.
\end{abstract}




\section{Conteúdo}

1 Introdução $\quad 3$

$\begin{array}{lll}2 & \text { Equação da Vorticidade } & 7\end{array}$

2.1 Equação de Euler . . . . . . . . . . . . . . . . . . . . 8

2.2 Equação da Vorticidade . . . . . . . . . . . . . . . . . . 15

2.3 Alguns resultados sobre circulação . . . . . . . . . . . . . . . . . 23

3 O modelo dos vórtices sem massa $\quad 27$

3.1 Vórtices puntuais: modelo de Helmholtz-Kirchhoff . . . . . . . . . . . 28

$3.2 \mathrm{O}$ modelo dos vórtices sem massa como um modelo Hamiltoniano . . . . 30

3.3 Partículas carregadas num campo magnético e sua relação com o modelo dos vórtices . . . . . . . . . . . . . . . . . . 37

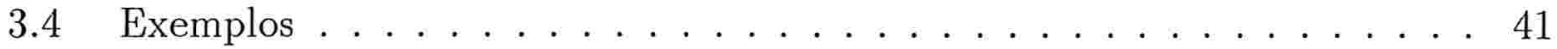

3.4.1 Exemplo1: dois vórtices sem massa . . . . . . . . . . . . . . . . 41

3.4.2 Exemplo 2 : três vórtices sem massa . . . . . . . . . . . . . . . 44

3.4.3 Exemplo 3 : quatro vórtices sem massa . . . . . . . . . . . . 51 
4.1 O modelo dos vórtices com massa como um sistema Hamiltoniano . . . . . 64

4.2 Exemplo 4: dois vórtices com massa, $\Gamma_{1}+\Gamma_{2} \neq 0 \ldots \ldots$

4.2.1 Redução para quatro variáveis . . . . . . . . . . . . . 66

4.2 .2 Um caso integrável $: \frac{m_{1}}{\Gamma_{1}}=\frac{m_{2}}{\Gamma_{2}} \ldots \ldots \ldots 72$

4.2 .3 Estabilidade . . . . . . . . . . . . . . . . . . . . 74

4.3 Exemplo 5: dois vórtices com massa, $\Gamma_{1}+\Gamma_{2}=0 \ldots \ldots \ldots$

4.3.1 Redução para quatro variáveis . . . . . . . . . . . . . 75

4.3.2 Um caso não integrável: $m_{1}=m_{2}=m \ldots \ldots \ldots$. . . . . 78

5 Modelo misto dos vórtices $\quad 81$

5.1 Variedades de Poisson . . . . . . . . . . . . . . . . . 82

5.2 Método de Dirac . . . . . . . . . . . . . . . . . . . 87

5.3 O modelo misto dos vórtices f . . . . . . . . . . . . . . . . 94

5.4 Exemplo 6: dois vórtices, um com massa e outro sem . . . . . . . . . . 99 


\section{Capítulo 1}

\section{Introdução}

O objetivo deste trabalho é estudar um modelo da física matemática, o modelo dos vórtices. Este modelo descreve duas situações físicas comuns em aplicações. A primeira é a de um fluido bidimensional, ou seja, um fluido ideal (sem viscosidade) e incompressível (densidade de massa constante) com o movimento essencialmente plano, em que a vorticidade esteja concentrada em alguns pontos. A motivação para a investigação do comportamento destes fluidos surge de observações experimentais de oceanos e atmosferas, que mostram a existência de zonas onde a vorticidade se encontra especialmente concentrada. A segunda é a de um conjunto de $N$ colunas paralelas de cargas, em um plasma, onde também foi observada concentração de vorticidade (ver [9]). O estudo de vórtices puntuais num fluido bidimensional foi iniciado por Helmholtz e Kirchhoff (ver [11]). Eles obtiveram as equações que governam o movimento de $N$ vórtices puntuais, sem massa, no plano, assumindo que cada vórtice não se movimenta por ação de seu campo, mas sim por ação do campo de velocidade gerado pelos outros vórtices.

As equações de Helmholtz e Kirchhoff, que governam o movimento de $N$ vórtices puntuais, são extendidas ao caso em que a cada vórtice está associada uma massa. Essa massa pode ser interpretada como a massa de uma impureza do fluido onde o campo de vorticidade é não nulo. Obtem-se assim o modelo dos vórtices com massa. Estas 
equações também determinam o comportamento de $N$ cargas bidimensionais, as quais podem ser vistas como a interseção de colunas infinitas de partículas carregadas com um plano ortogonal, sujeitas a um campo magnético constante. Pode-se mostrar que tanto o modelo dos vórtices com massa quanto o modelo dos vórtices sem massa são sistemas Hamiltonianos. Entretanto, não é possivel passar de um ao outro tomando-se simplesmente as massas nulas, pois a Hamiltoniana do modelo dos vórtices com massa se torna singular nesta situação. Por outro lado, existe um procedimento que nos permite reduzir o sistema dos vórtices com massa à uma sub-variedade de seu espaço de fase, onde a Hamiltoniana não se torna singular quando uma das massas se anula. Desta forma, é obtido o modelo misto dos vórtices, isto é, o modelo no qual alguns dos vórtices não têm massa. O modelo misto dos vórtices também será um sistema Hamiltoniano.

Quando se trata de sistemas Hamiltonianos, uma questão de extrema importância é a da integrabilidade no sentido de Liouville. Os sistemas integráveis são raros, mas sua dinâmica é a única que se pode entender completamente, já que comportamentos caóticos estão excluídos. Nos três modelos tratados aqui existem duas integrais primeiras em involução, independentes da Hamiltoniana. Isto é suficiente para garantir a integrabilidade do sistema de dois ou três vórtices sem massa, e de um vórtice com massa e outro sem massa. Entretanto, pode-se mostrar que os sistemas com quatro vórtices sem massa, com dois vórtices com massa e com um vórtice com massa e dois sem massa são não integráveis e apresentam, para certos valores dos parâmetros, comportamento caótico (ver [5], [13], [25]).

Este trabalho tem a seguinte organização. No capítulo 2, são obtidas heuristicamente as equações que determinam completamente o fluxo gerado pelo campo da vorticidade, num domínio $U$ simplemente conexo de $\mathbb{R}^{2}$. Estas equações são deduzidas a partir das equações de Euler que regem o movimento de um fluido ideal e incompressível, confinado a um domínio $U$ no plano. No capítulo 3 estuda-se o modelo dos vórtices sem massa. Na seção 3.1 é apresentado o modelo dos vórtices baseado na hipótese de Helmholtz e Kirchhoff (ver [11]). Este modelo é obtido a partir da equação de vorticidade, supondo 
que ela está concentrada num número finito de ponto $\vec{x}_{1}, \cdots, \vec{x}_{N}$, em $U \subset \mathbb{R}^{2}$. Na seção 3.2 mostraremos que o modelo é um sistema Hamiltoniano. Na seção 3.3 ligaremos este modelo com o das cargas bidimensionais. Finalmente, na seção 3.4 estudaremos o problema de dois, três e quatro vórtices sem massa, para diferentes valores das intensidades. Nos dois primeiros casos o sistema é integrável no sentido Liouville (ver [2] ou [21]). O movimento dos três vórtices é completamente analizado em [27]. No terceiro caso, consideramos o problema de quatro vótices, com intensidade $(1,1,1, \varepsilon)$, como uma perturbação do problema de três vórtices com intensidade $(1,1,1)$, o qual admite conexões de selas. Para $\varepsilon>0$, suficientemente pequeno, o problema perturbado é reduzido a um sistema Hamiltoniano plano que não é mais autônomo, mas depende periódicamente do tempo. Foi possível mostrar, mediante o método de Melnikov (ver [15]), a existência de comportamento caótico (ver [5]). Isto implica que há novas regiões de comportamento caótico diferentes das encontradas previamente por Ziglin (ver [29]).

No capítulo 4, seção 4.1, definimos as equações de movimento dos vórtices com massa a partir da analogia eletromagnética e do modelo físico para a força que age num vórtice com massa (ver [11]). Na seção 4.2, estuda-se o sistema de dois vórtices com massa, no caso em que a soma de suas intensidades é não nula (ver [25]). Utiliza-se as integrais primeiras do sistema, para a redução de quatro a dois graus de liberdade. No caso particular em que razão entre massa e intensidade forem iguais para os dois vórtices o sistema apresenta mais uma integral primeira independente das anteriores, e é portanto integrável. É possivel mostrar que algumas das órbitas deste sistema ficam próximas de órbitas do sistema de dois vórtices sem massa, quando as massa tendem a zero. Na seção 4.3 é estudado um sistema de dois vórtices com massa no caso da soma de suas intensidades se anular. Novamente, utiliza-se as integrais primeiras para reduzir o sistema a dois graus de liberdade. Neste caso, quando as duas massas forem iguais, é possível mostrar (ver [25]) que o sistema é não integrável.

Finalmente, no capítulo 5, estuda-se o modelo misto dos vórtices. Na secção 5.1 apresenta-se os conceitos e propriedades de variedades de Poisson, que é uma generali- 
zação de variedades simpléticas. Na seção 5.2 apresenta-se o método de Dirac, que permite, sob certas condições, restringir um sistema Hamiltoniano numa variedade simplética a um sistema hamiltoniano numa subvariedade onde está bem definida uma estrutura de Poisson (ver [4]). Na seção 5.3, aplica-se o formalismo de Dirac para obter o modelo misto dos vórtices a partir da restrição do modelo dos vórtices com massa a uma subvariedade conveniente de seu espaço de fase. Esta subvariedade é escolhida de forma que a Hamiltoniana associada ao sistema de vórtices com massa não se torne singular quando fazemos as massas tender para zero. Na seção 5.4, estudaremos o sistema misto de dois vórtices, que é completamente integrável, independentemente do valor das intensidades e da massa, por possuir duas integrais primeiras em involução, além da energia. Entretanto, em certos casos é possível mostrar que o sistema com dois vórtices sem massa e um com massa não é integrável (ver [12]). 


\section{Capítulo 2}

\section{Equação da Vorticidade}

Neste capítulo vamos expor algumas idéias básicas sobre a mecânica de um fluido ideal. Nosso objetivo é apresentar heuristicamente as equações que regem este fenômeno, no caso especialmente importante de um fluido ideal (sem viscosidade) e incompressível (densidade de massa constante), numa região do espaço. Estas equações servirão como base para a discussão do modelo dos vórtices, apresentado no próximo capítulo. Na seção 2.1 começaremos deduzindo as equações do movimento de um fluido ideal, que correspondem às equações de Euler. Estas equações serão derivadas a partir das leis de conservação da massa e momento, e das hipóteses constitutivas do fluido.

Na seção 2.2 apresentaremos inicialmente as equações para o campo de vorticidade de um fluido no caso especial em que a densidade de massa é constante e que a força é derivada de um potencial. Em seguida, supondo que o movimento do fluido é essencialmente plano, mostraremos que a vorticidade pode ser descrita por um campo escalar e que o campo de velocidades pode ser escrito em termos da chamada função de corrente (escalar). Apresentaremos, finalmente, as equações que determinam o movimento do fluido neste caso particular, em termos da função de corrente e do campo escalar de vorticidade.

Na seção 2.3, apresentaremos alguns resultados clássicos sobre circulação, que nos permitirão concluir que o fluxo da vorticidade através de uma superfície movimentando- 
se com o fluido é constante no tempo.

\subsection{Equação de Euler}

$\mathrm{Na}$ presente seção introduziremos algumas idéias básicas acerca do movimento de um fluido ideal, do ponto de vista de espaços Euclidianos, isto é, os campos vetoriais e os fluxos gerado por estes campos são definidos sobre um domínio de $\mathbb{R}^{3}$, e serão estudados usando as ferramentas do cálculo vetorial (ver [7] e [16]). Embora nesta dissertação só tratemos de fluidos definidos em domínios de $\mathbb{R}^{3}$, estes resultados podem ser generalizados para o contexto de variedades e formas diferenciáveis (ver [2]).

Observação 2.1 As expressões região e domínio serão usadas para designar conjuntos abertos e conexos. As componente de um vetor $\vec{v}$ serão indicadas indistintamente por $\left(v_{1}, v_{2}, v_{3}\right)$ ou por $\left(v_{x}, v_{y}, v_{z}\right)$, conforme for mais conveniente.

Um "estado" de um fluido em movimento confinado à região $D$ de $\mathbb{R}^{3}$ é descrito matematicamente por um campo vetorial $\vec{u}$ definido em $\mathrm{D}$, chamado campo de velocidades do fluido. Seja $\vec{x}$ um ponto em $D$ e imaginemos uma partícula no fluido (por exemplo uma partícula de pó suspensa no fuido), que se encontra no ponto $\vec{x}$ no tempo $t=0$. Esta partícula descreve uma trajetória, que denotaremos por $\varphi_{\vec{x}}(t)=\varphi(\vec{x}, t)$.

Observação 2.2 Se o movimento da partícula é determinado pelo campo de velocidades do fluido, então sua trajetória satisfaz a equação diferencial:

$$
\frac{d \varphi_{\vec{x}}(t)}{d t}=\vec{u}\left(\varphi_{\vec{x}}(t), t\right)
$$

e a condição inicial $\varphi_{\vec{x}}(0)=\vec{x}$. Note que esta equação implica que a trajetória é tangente ao campo de velocidades.

É portanto natural definir : 
Definição 2.1 O fluxo gerado pelo campo vetorial $\vec{u}$ é a aplicação $\varphi: D \times \mathbb{R} \rightarrow \mathrm{D}$ tal que $\varphi_{\vec{x}}(t)=\varphi(\vec{x}, t)$ satisfaz $(2.1)$ para todo $\vec{x}$ em $D$, e tal que $\varphi_{t}: D \rightarrow D$ definida por $\varphi_{t}(\vec{x})=\varphi(\vec{x}, t)$ satisfaz $\varphi_{o}=$ identidade. Qualquer uma das aplicações $\varphi_{\vec{x}}: \mathbb{R} \rightarrow \mathrm{D}$, com $\vec{x} \in D$, é chamada uma trajetória do fluido.

Observação 2.3 No caso em que $\vec{u}$ e $D$ são arbitrários, a aplicação $\varphi_{\vec{x}}$ só estará definida para $t$ variando num intervalo, dependente de $\vec{x}$. Entretanto, no caso do movimento do fluido, é fisicamente impossivel que este intervalo seja finito, para algum $\vec{x}$. Estaremos supondo então que $\vec{u}$ e $D$ satisfaçam condições suficientes para que o fluxo esteja definido para todo $t \in \mathbb{R}$, e que $\varphi_{t}: D \rightarrow D$ seja um difeomorfismo, para todo $t \in \mathbb{R}$.

Outro conceito importante na descrição do movimento do fluido é o de linha de corrente:

Definição 2.2 Dado o campo de velocidade $\vec{u}(\vec{x}, t)$ de um fluido, uma linha de corrente, definida para um certo tempo fixo $t$ é uma curva integral de $\vec{u}_{t}(\vec{x})=\vec{u}(\vec{x}, t)$; isto é, se $\vec{x}(s), s \in \mathbb{R}$ é uma parametrização desta linha de corrente, então satisfaz:

$$
\vec{x}^{\prime}(s)=\vec{u}(\vec{x}(s), t) \quad \forall s \in \mathbb{R}
$$

Observação 2.4 As linhas de corrente corresponderiam a trajetórias num fluido cujo campo de velocidades fosse $\vec{u}(\cdot, t)$, com $t$ fixo. Note então que, se $\vec{u}$ é independente do tempo $t$, as linhas de corrente e as trajetórias coincidem. Neste caso o fluido é chamado estacionário.

Para cada tempo t, assumamos que é bem definida a densidade de massa do fluido, denotada $\rho_{t}(\vec{x})=\rho(\vec{x}, t)$. Assim, se $W$ é qualquer sub-região de $D$, a massa do fluido em $W$, no tempo $t$, é dada por:

$$
m(W, t)=\int_{W} \rho(\vec{x}, t) d V
$$


onde $d V$ é o elemento de volume no plano ou no espaço.

No que segue assumiremos que as funções dadas $\vec{u}$ e $\rho$ são suficientemente suaves, de forma que possamos aplicar as operações do cálculo diferencial necessárias.

A derivação das equações é fundamentada em três partes distintas:

(i) conservação da massa;

(ii) hipóteses constitutivas: fluido incompressível;

(iii) hipóteses constitutivas: fluido ideal e balanço de momento.

\section{(i) Conservação da massa.}

Este princípio nos diz que a massa do fluido, que no tempo $\mathrm{t}=0$ ocupa uma região $W$, permanece inalterada depois de um certo tempo. Mais precisamente, se $W$ é uma região de $D$ com fronteira suficientemente suave para que o Teorema de Stokes seja válido, então

$$
\int_{W_{t}} \rho(\vec{x}, t) d V=\int_{W} \rho(\vec{x}, 0) d V
$$

onde $W_{t}=\varphi_{t}(W)$. Usando a fórmula de mudança de variáveis, a conservação da massa (2.4) pode ser reescrita como

$$
\int_{W} \rho\left(\varphi_{t}(\vec{x}), t\right) J(\vec{x}, t) d V=\int_{W} \rho(\vec{x}, 0) d V
$$

onde $J(\vec{x}, t)$ é o jacobiano de $\varphi_{t}$. Como $W$ é arbitrário, tem-se que

$$
\rho\left(\varphi_{t}(\vec{x}), t\right) J(\vec{x}, t)=\rho(\vec{x}, 0)
$$

Em seguida, apresentaremos um lema que nos será útil para obter a equação da conservação da massa, e para a caracterização de fluidos incompressíveis.

Lema 2.1.1 Se $J(\vec{x}, t)$ é o jacobiano de $\varphi_{t}$, temos que 


$$
\frac{\partial}{\partial t} J(\vec{x}, t)=J(\vec{x}, t)\left[\operatorname{div}\left(\vec{u}\left(\varphi_{t}(\vec{x}), t\right)\right]\right.
$$

Demostração. Ver [7] .

Derivando (2.5) com respeito a t obtemos, a partir de (2.1) e do lema anterior, que

$$
\frac{\partial \rho}{\partial t}+\operatorname{div}(\rho \vec{u})=0
$$

Esta última equação é a forma diferencial da lei da conservação de massa, também conhecida como equação de continuidade.

\section{(ii) Hipóteses constitutivas: fluido incompressível}

A descrição do movimento de um corpo ou fluido contínuo ( cinemática do contínuo) usando as ferrramentas do cálculo diferencial não é suficiente para determinar completamente a evolução no tempo deste corpo (dinâmica). Se queremos esta dinâmica, precisamos fazer hipóteses adicionais que levem em conta as diferenças encontradas nos diversos materiais (por exemplo, o comportamento de uma porção de óleo numa garrafa é completamente diferente daquele de uma porção de gás numa garrafa semelhante). Estas hipóteses são chamadas hipóteses constitutivas . Consideraremos dois tipos de hipóteses constitutivas sobre o fluido:

- hipóteses sobre o tipo de deformação que o corpo pode sofrer, como por exemplo se um corpo é rígido ou incompressível. Nos assumiremos a hipótese que o fluido é incompressível, o que tem se mostrado uma hipótese bastante realista para líquidos como a água, em condições normais de fluxo;

- hipóteses sobre a forma das forças (internas) que agem em cada parte do corpo ou fluido. Nós adotaremos a hipótese que o fluido é ideal, o que tem se mostrado uma hipótese realista quando os efeitos viscosos são desprezíveis.

Definição 2.3 Um fluido é dito incompressivel se para qualquer região $W_{t}=\varphi(W, t)$ 
ocupada pelo fluido no tempo $t$, tem-se

$$
\operatorname{Volume}\left(W_{t}\right)=\int_{W_{t}} d V=\text { constante em } t .
$$

Note que se o fluido é incompressível então, usando o lema (2.1.1) e o teorema de mudança de variáveis, que para toda região $W_{t}=\varphi(W, t)$, com fronteira suave, deslocando-se com o fluido tem-se que:

$$
\begin{aligned}
0 & =\frac{d}{d t} \int_{W_{t}} d V=\frac{d}{d t} \int_{W} \mathbf{J} d V=\int_{W} \dot{\mathbf{J}} d V= \\
& =\int_{W} \mathbf{J} \operatorname{div} \vec{u} d V=\int_{W_{t}} \operatorname{div} \vec{u} d V=\int_{\partial W_{t}} \vec{u} \cdot \hat{\mathbf{n}} d A .
\end{aligned}
$$

Dos resultados acima, e do fato de $\mathbf{J}$ não se anular, temos o seguinte teorema:

Teorema 2.1.1 (Caracterização de fluidos incompressíveis) As seguintes condições são equivalentes:

1- o fluido é incompressível;

2- $\operatorname{div} \vec{u}=0$;

3- $\mathbf{J}=1$;

4- Para todo $W_{t}$ como acima temos $\int_{\partial W_{t}} \vec{u} \cdot \hat{\mathbf{n}} d A=0$.

\section{(iii) Hipóteses constitutivas: fluido ideal e balanço de momento}

Na mecânica dos fluidos postula-se que as forças envolvidas no movimento são descritas por duas funções suaves: b e s. A função b representa a força que o meio exerce intrinsicamente sobre as partículas do fluido, sendo o exemplo mais clássico a força gravitacional. Ou seja, b : $D \times \mathbb{R} \rightarrow \mathbb{R}^{3}$ é uma função suficientemente diferenciável e b $(\vec{x}, t)$ é a força, por unidade de massa, exercida pelo meio no ponto $\vec{x}$, no instante $t$.

A função s : $S^{2} \times D \times \mathbb{R} \rightarrow \mathbb{R}^{3}$ descreve a força resultante de uma parte do fluido sobre outra. Se $W \subset D$ é uma região fechada, orientada, com fronteira suave e $W_{t}=\varphi(W, t)$, seja $\vec{x} \in \partial W_{t}$ e $\vec{n}(\vec{x}, t)$ a normal externa unitária a $W_{t}$, no ponto $\vec{x}$, no 
instante $t$. Então $\mathrm{s}(\vec{n}(\vec{x}, t), \vec{x}, t)$ é a força superficial, por unidade de área, exercida em $\vec{x}$, no instante $t$. A força total que age sobre $W_{t}$ no instante $t$ é portanto:

$$
\mathbf{F}(W, t)=\int_{\partial W_{t}} \mathbf{s}(\vec{n}(\vec{x}, t), \vec{x}, t) d A+\int_{W_{t}} \rho(\vec{x}, t) \mathbf{b}(\vec{x}, t) d V .
$$

Podemos agora definir:

Definição 2.4 Um fluido é ideal se $\mathrm{s}(\vec{n}, \vec{x}, t)=-p(\vec{x}, t) \vec{n}$. A função $p: D \times \mathbb{R} \rightarrow \mathbb{R}$ é chamada de pressão.

Aplicando o teorema do divergente em (2.9), obtemos que no fluido ideal a força é dada por:

$$
\mathbf{F}(W, t)=\int_{W_{t}}(\rho \mathbf{b}-\operatorname{grad} p)(\vec{x}, t) d V
$$

e, pelo teorema de mudanca de variáveis,

$$
\mathbf{F}(W, t)=\int_{W}(\rho \mathbf{b}-\operatorname{grad} p)(\varphi(\vec{x}, t), t) J(\vec{x}, t) d V
$$

A segunda lei de Newton (balanço de momento) assegura que a taxa de variação do momento de uma porção do fluido é igual ao total da força nela aplicada. Ou seja, se $\mathrm{l}(W, t)$ é o momento do fluido contido em $W_{t}$ no instante $t$, então

$$
\frac{d}{d t}(\mathbf{l}(W, t))=\mathbf{F}(W, t)
$$

Mas, pelo teorema de mudança de variável,

$$
\frac{d}{d t}(\mathrm{l}(W, t))=\frac{d}{d t} \int_{W_{t}}(\rho \vec{u})(\vec{x}, t) d V=\frac{d}{d t} \int_{W}(\rho \vec{u})(\varphi(\vec{x}, t), t) J(\vec{x}, t) d V,
$$

e da conservação da massa (equação 2.5), obtemos:

$$
\frac{d}{d t}(\mathrm{l}(W, t))=\int_{W} \rho(\vec{x}, 0) \frac{d}{d t} \vec{u}(\varphi(\vec{x}, t), t) d V .
$$


Observação 2.5 Se $\varphi_{\vec{x}}(t), t \in \mathbb{R}$ é uma trajetória do fluido e $\vec{w}: D \times \mathbb{R} \rightarrow \mathbb{R}^{3}$ é uma função diferenciável, então, pela regra da cadeia, temos:

$$
\frac{d}{d t}\left(w_{i}\left(\varphi_{\vec{x}}(t), t\right)\right)=\sum_{j=1}^{3}\left(\frac{\partial w_{i}}{\partial x_{j}} \frac{\partial \varphi_{j}}{\partial t}\right)+\frac{\partial w_{i}}{\partial t}=\left(\sum_{j=1}^{3} \frac{\partial w_{i}}{\partial x_{j}} u_{j}+\frac{\partial w_{i}}{\partial t}\right)\left(\varphi_{\vec{x}}(t), t\right) .
$$

Se denotarmos $\vec{u} \cdot \nabla=u_{1} \frac{\partial}{\partial x_{1}}+u_{2} \frac{\partial}{\partial x_{2}}+u_{3} \frac{\partial}{\partial x_{3}}$, teremos que

$$
\frac{d}{d t}\left(\vec{w}\left(\varphi_{\vec{x}}(t), t\right)\right)=\left(\frac{\partial}{\partial t}+\vec{u} \cdot \nabla\right) \vec{w}\left(\varphi_{\vec{x}}(t), t\right)
$$

Definição 2.5 A derivada material é o operador linear

$$
\frac{D}{D t}=\frac{\partial}{\partial t}+\vec{u} \cdot \nabla
$$

definido nas funções diferenciáveis com domínio $D \times \mathbb{R}$ e assumindo valores em $\mathbb{R}^{3}$ ou $\mathbb{R}$.

Com a observação e definição acima, a variação de momento pode ser escrita como:

$$
\frac{d}{d t}(\mathrm{l}(W, t))=\int_{W} \rho(\vec{x}, 0) \frac{D \vec{u}}{D t}(\varphi(\vec{x}, t), t) d V .
$$

Como o fluido é incompressível temos, pelo teorema 2.1.1, que:

$$
\mathbf{F}(W, t)=\int_{W}(\rho \mathbf{b}-\operatorname{grad} p)\left(\varphi_{t}(\vec{x}), t\right) d V,
$$

e como $W$ é arbitrário em (2.16) e (2.17) obtemos, a partir de (2.12), a forma diferencial da equação do balanço de momento:

$$
\rho\left(\varphi_{t}(\vec{x}), t\right) \frac{D \vec{u}}{D t}\left(\varphi_{t}(\vec{x}), t\right)=(\rho \mathbf{b}-\operatorname{grad} p)\left(\varphi_{t}(\vec{x}), t\right),
$$

para todo $\vec{x} \in D, t \in \mathbb{R}$. Como $\varphi_{t}$ é um difeomorfismo em $\mathrm{D}$, a equação acima vale em todos os pontos de D. Obtemos assim a equação de Euler do fluido ideal:

$$
\rho \frac{D \vec{u}}{D t}=-\operatorname{grad} p+\rho \mathbf{b}
$$


Observação 2.6 As condiçoẽs de fronteiras sobre as equações são impostas a partir do problema físico que se está modelando. Se queremos o fluido confinado a uma região $D$ de $\mathbb{R}^{3}$, deveremos impor que o campo de velocidade é tangente ao bordo $\partial D$, ou seja, o fluido não atravessa o bordo $\partial D$.

Observação 2.7 Note que se o fluido é incompressivel, a equação da continuidade (2.7) pode ser rescrita como:

$$
\frac{\partial \rho}{\partial t}+\operatorname{div}(\rho \vec{u})=\frac{\partial \rho}{\partial t}+\vec{u} \cdot \operatorname{grad} \rho+\rho \operatorname{div} \vec{u}=\frac{D \rho}{D t}=0 .
$$

Temos portanto, usando as notações anteriores, que as equações de movimento de um fluido ideal e incompressível, num domínio $D$ de $\mathbb{R}^{3}$ são:

equação de Euler:

$$
\rho \frac{D \vec{u}}{D t}=-\operatorname{grad} p+\rho \mathbf{b}
$$

equação de continuidade:

$$
\frac{D \rho}{D t}=0
$$

condição de incompressibilidade:

$$
\operatorname{div} \vec{u}=0
$$

e condição de fronteira:

$$
\vec{u} \cdot \hat{\mathbf{n}}=0 \text { em } \partial D
$$

\subsection{Equação da Vorticidade}

O modelo dos vórtices não é dado em termos do campo de velocidades, mas sim do campo de vorticidade, definido por: 
Definição 2.6 Se $\vec{u}$ é o campo de velocidade de um fluido em $D \subset \mathbb{R}^{3}$, então

$$
\vec{\Omega}=\nabla \times \vec{u}=\left(\frac{\partial u_{z}}{\partial y}-\frac{\partial u_{y}}{\partial z}, \frac{\partial u_{x}}{\partial z}-\frac{\partial u_{z}}{\partial x}, \frac{\partial u_{y}}{\partial x}-\frac{\partial u_{x}}{\partial y}\right)
$$

é chamado campo de vorticidade do fluxo. Em outras palavras, o campo de vorticidade é o rotacional do campo de velocidade do fluido.

No que segue, vamos fazer duas hipóteses adicionais:

i) vamos supor que o fluido é homogêneo, o que é equivalente, pela condição de incompressibilidade, a $\rho(\vec{x}, 0)=\rho=$ constante. Teremos então que $\rho(\vec{x}, t)=\rho$ para todo $\vec{x} \in D$ e $t \in \mathbb{R}$;

ii) vamos supor ainda que a força b seja derivada de um potencial, isto é, que exista uma função $\Phi: D \times \mathbb{R} \rightarrow \mathbb{R}$ tal que $\mathbf{b}=\operatorname{grad} \Phi$.

Nestas condições, a equação de Euler, em termos do campo de vorticidade, é dada na sequinte proposição:

Proposição 2.2.1 Seja $D \subset \mathbb{R}^{3}$, com $\partial D$ superfície lisa . Então :

$$
\frac{D \vec{\Omega}}{D t}=(\vec{\Omega} \cdot \vec{\nabla}) \vec{u}
$$

e

$$
\vec{\Omega}(\varphi(\vec{x}, t), t)=\vec{\nabla} \varphi_{t}(\vec{x}) \cdot \vec{\Omega}(\vec{x}, 0)
$$

onde $\vec{\nabla} \varphi_{t}$ é a diferencial de $\varphi_{t}$ (matriz Jacobiana), e $\vec{\Omega} \cdot \vec{\nabla}=\Omega_{x} \frac{\partial}{\partial x}+\Omega_{y} \frac{\partial}{\partial y}+\Omega_{z} \frac{\partial}{\partial z}$.

\section{Demonstração:}

Aplicando o rotacional ( $\vec{\nabla} \times)$ à expressão (2.19), obtemos

$$
\vec{\nabla} \times \frac{D}{D t} \vec{u}=\frac{\partial}{\partial t}(\vec{\nabla} \times \vec{u})+\vec{\nabla} \times((\vec{u} \cdot \vec{\nabla}) \vec{u})=\vec{\nabla} \times \operatorname{grad}\left(-\frac{p}{\rho}+\Phi\right)=0 .
$$

Utilizando a identidade vetorial

$$
\frac{1}{2} \vec{\nabla}(\vec{u} \cdot \vec{u})=\vec{u} \times(\vec{\nabla} \times \vec{u})+(\vec{u} \cdot \vec{\nabla}) \vec{u}
$$


a equação (2.26) fica

$$
\frac{\partial}{\partial t}(\vec{\nabla} \times \vec{u})+\vec{\nabla} \times\left[\frac{1}{2} \vec{\nabla}(\vec{u} \cdot \vec{u})-\vec{u} \times(\vec{\nabla} \times \vec{u})\right]=0 .
$$

Simplificando e fazendo $\vec{\Omega}=\vec{\nabla} \times \vec{u}$, obtemos

$$
\frac{\partial \vec{\Omega}}{\partial t}-\vec{\nabla} \times(\vec{u} \times \vec{\Omega})=0 .
$$

Se notarmos que $\vec{\nabla} \cdot \vec{\Omega}=0$ e considerarmos a identidade vetorial

$$
\vec{\nabla} \times(\vec{u} \times \vec{\Omega})=(\vec{\Omega} \cdot \vec{\nabla}) \vec{u}-\vec{\Omega}(\vec{\nabla} \cdot \vec{u})-(\vec{u} \cdot \vec{\nabla}) \vec{\Omega}+\vec{u}(\vec{\nabla} \cdot \vec{\Omega})
$$

obtemos, a partir de (2.27), que

$$
\frac{\partial \vec{\Omega}}{\partial t}+(\vec{u} \cdot \vec{\nabla}) \vec{\Omega}=(\vec{\Omega} \cdot \vec{\nabla}) \vec{u},
$$

ou seja,

$$
\frac{D \vec{\Omega}}{D t}=(\vec{\Omega} \cdot \vec{\nabla}) \vec{u}
$$

Para demonstrar (2.25), definamos

$$
\begin{gathered}
\vec{F}(\vec{x}, t)=\vec{\Omega}(\varphi(\vec{x}, t), t), \\
\vec{G}(\vec{x}, t)=\vec{\nabla} \varphi(\vec{x}, t) \cdot \vec{\Omega}(\vec{x}, 0)) .
\end{gathered}
$$

Note que, como $\varphi(\vec{x}, 0)=\vec{x}$, as duas funções coincidem em $t=0$. Derivando (2.29) com relação ao tempo temos, a partir de (2.24) e de (2.14), que

$$
\frac{\partial \vec{F}}{\partial t}(\vec{x}, t)=((\vec{F} \cdot \nabla) \vec{u})(\varphi(\vec{x}, t), t) .
$$

Por outro lado, derivando a expressão (2.30) com relação ao tempo, tem-se:

$$
\left.\frac{\partial \vec{G}}{\partial t}=\frac{\partial \vec{\nabla} \varphi(\vec{x}, t)}{\partial t} \cdot \vec{\Omega}(\vec{x}, 0)\right)
$$


Em coordenadas, temos que:

$$
\begin{aligned}
\left(\frac{\partial \vec{\nabla} \varphi(\vec{x}, t)}{\partial t}\right)_{i j} & =\frac{\partial}{\partial t} \frac{\partial \varphi_{i}(\vec{x}, t)}{\partial x_{j}}= \\
& =\frac{\partial}{\partial x_{j}}\left(\frac{\partial \varphi_{i}(\vec{x}, t)}{\partial t}\right)= \\
& =\frac{\partial}{\partial x_{j}} u_{i}(\varphi(\vec{x}, t), t)= \\
& =\sum_{k=1}^{3} \frac{\partial u_{i}}{\partial x_{k}}(\varphi(\vec{x}, t), t) \frac{\partial \varphi_{k}}{\partial x_{j}}(\vec{x}, t) .
\end{aligned}
$$

Portanto, a partir de (2.32), temos que

$$
\begin{aligned}
\frac{\partial G_{i}}{\partial t}(\vec{x}, t) & \left.=\sum_{j=1}^{3} \sum_{k=1}^{3} \frac{\partial u_{i}}{\partial x_{k}}(\varphi(\vec{x}, t), t) \frac{\partial \varphi_{k}}{\partial x_{j}}(\vec{x}, t) \Omega_{j}(\vec{x}, t), t\right)= \\
& =\left[\left(\sum_{k=1}^{3}\left(\sum_{j=1}^{3} \frac{\partial \varphi_{k}}{\partial x_{j}}(\vec{x}, t) \Omega_{j}(\vec{x}, t)\right) \frac{\partial}{\partial x_{k}}\right) u_{i}\right](\varphi(\vec{x}, t), t)= \\
& =\left[\left(\sum_{k=1} G_{k}(\vec{x}, t) \frac{\partial}{\partial x_{k}}\right) u_{i}\right](\varphi(\vec{x}, t), t)= \\
& =\left((\vec{G}(\vec{x}, t) \cdot \nabla) u_{i}\right)(\varphi(\vec{x}, t), t),
\end{aligned}
$$

isto é,

$$
\frac{\partial \vec{G}}{\partial t}(\vec{x}, t)=((\vec{G}(\vec{x}, t) \cdot \nabla) \vec{u})(\varphi(\vec{x}, t), t) .
$$

Assim, a partir de (2.31) e (2.33), temos que $\vec{F}$ e $\vec{G}$ satisfazem a mesma equação diferencial de primeira ordem, e como elas coincidem para $t=0$, tem-se que são iguais. Notemos que a expresão (2.25) mostra que a vorticidade evolui no tempo por meio da aplicação fluxo $\varphi_{t}(\vec{x})=\varphi(\vec{x}, t)$, gerada pelo campo vetorial $\vec{u}$.

Vamos agora considerar o caso em que o fluido esteja restrito a mover-se paralelamente a um plano, com velocidades dependentes apenas da projeção do ponto neste plano. Matematicamente, estamos supondo que exista um sistema de coordenadas onde $D=$ $U \times(a, b)$ e o campo de velocidade seja dado por

$$
\vec{u}(\vec{x}, t)=\vec{u}(x, y, z, t)=\left(u_{x}(x, y, t), u_{y}(x, y, t), 0\right), \quad \forall(x, y) \in U, z \in(a, b), t \in \mathbb{R} .
$$


Observação 2.8 Note que, pela equação (2.1), a terceira componente do fluxo $\varphi$ é constante no tempo, donde $\varphi_{z}(x, y, z, t)=\varphi_{z}(x, y, z, 0)=z$. Portanto, fixando $z=z_{0} \in(a, b)$, matematicamente passamos a ter um problema bidimensional nas variáveis espaciais (que deverão pertencer a $U$ ), e cujos campos vetoriais relevantes assumem valores em $\mathbb{R}^{2}$ quando este é identificado com $\left\{\left(x, y, z_{0}\right), x, y \in \mathbb{R}\right\} \subset \mathbb{R}^{3}$ ou com $\{(x, y, 0), x, y \in$ $\mathbb{R}\} \subset \mathbb{R}^{3}$. Quando não causar confusão, no que segue, usaremos $\vec{x}$ indistintamente para indicar $\left(x, y, z_{0}\right) \in \mathbb{R}^{3}$ ou $(x, y) \in \mathbb{R}^{2}, \vec{u}$ para indicar $\left(u_{x}, u_{y}, 0\right) \in \mathbb{R}^{3}$ ou $\left(u_{x}, u_{y}\right) \in \mathbb{R}^{2}$ e $\vec{\varphi}$ para indicar $\left(\varphi_{x}, \varphi_{y}, z_{0}\right)$ ou $\left(\varphi_{x}, \varphi_{y}\right)$. Note ainda que o teorema 2.1.1 implica, nestas condiçốes, que o jacobiano de $\varphi_{t}$ é 1 .

De acordo com (2.23), tem-se que o campo de vorticidade é da forma

$$
\vec{\Omega}(x, y, z, t)=\vec{\Omega}(x, y, t)=\left(0,0,\left(\frac{\partial u_{y}}{\partial x}-\frac{\partial u_{x}}{\partial y}\right)(x, y, t)\right)=(0,0, \xi(x, y, t)) .
$$

Portanto, o campo de vorticidade pode ser descrito por um campo escalar $\xi: U \times \mathbb{R} \rightarrow$ $\mathbb{R}$, uma vez que $\vec{\Omega}$ só tem uma componente não nula. Observe que sob estas condições, $\vec{\Omega} \cdot \nabla \vec{u}=0$, e portanto, a equação da vorticidade (2.24) pode ser reescrita como

$$
\frac{D \xi}{D t}=\frac{\partial \xi}{\partial t}+(\vec{u} \cdot \nabla) \xi=0
$$

A condição de incompressibilidade (2.21) garante que :

$$
\frac{\partial u_{x}}{\partial x}=-\frac{\partial u_{y}}{\partial y} .
$$

Se assumirmos que $D$ é simplemente conexo temos, a partir do cálculo vetorial ( para funções suficientemente suaves), que existe uma função $\Psi: U \times \mathbb{R} \rightarrow \mathbb{R}$, única a menos de uma constante aditiva (que depende, eventualmente, do tempo), tal que:

$$
\begin{aligned}
& \frac{\partial \Psi}{\partial x}=-u_{y}(x, y, t) \\
& \frac{\partial \Psi}{\partial y}=u_{x}(x, y, t) .
\end{aligned}
$$

Podemos então definir: 
Definição 2.7 Seja $U \subset \mathbb{R}^{2}$ um domínio simplesmente conexo. Uma função $\Psi: U \times$ $\mathbb{R} \rightarrow \mathbb{R}$ satifazendo (2.36) é chamada função de corrente.

Observemos que, para todo $t$ fixo, as linhas de corrente, definidas em (2.2), se situam nas curvas de nível de $\Psi$. De fato, se $(x(s), y(s))$ é uma linha de corrente, temos, utilizando (2.36), que:

$$
\begin{aligned}
\frac{\partial \Psi}{\partial s}(x(s), y(s), t) & =\frac{\partial \Psi}{\partial x}(x(s), y(s), t) x^{\prime}(s)+\frac{\partial \Psi}{\partial y}(x(s), y(s), t) y^{\prime}(s)= \\
& =\left(\frac{\partial \Psi}{\partial x} u_{x}+\frac{\partial \Psi}{\partial y} u_{y}\right)(x(s), y(s), t)= \\
& =\left(-u_{y} u_{x}+u_{y} u_{x}\right)(x(s), y(s), t)= \\
& =0
\end{aligned}
$$

No caso particular que estamos tratanto, o versor $\overrightarrow{\mathbf{n}}$, normal exterior a $\partial D$, é horizontal nos pontos da forma $(\vec{x}, c)$, com $\vec{x} \in \partial D, c \in(a, b)$, ou seja, $\overrightarrow{\mathbf{n}}=\left(n_{x}, n_{y}, 0\right)$, com $\left(n_{x}, n_{y}\right)$ (também denotado por $\overrightarrow{\mathbf{n}}$ ) normal exterior a $\partial U$. A condição de fronteira (2.22) é portanto equivalente a $\vec{u} \cdot \overrightarrow{\mathbf{n}}=0$, em $\partial U$. Podemos demonstrar então que:

Proposição 2.2.2 A fronteira $\partial U$ se situa nas curvas de nível de $\Psi(x, y, t)$.

\section{Demonstração:}

Se $(x(s), y(s))$ é uma parametrização local de $\partial U$, então $\left(-y^{\prime}(s), x^{\prime}(s)\right)$ é paralelo a $\vec{n}$. Mas

$$
\begin{aligned}
\frac{\partial \Psi}{\partial s}(x(s), y(s), t) & =-u_{y}(x(s), y(s), t) x^{\prime}(s)+u_{x}(x(s), y(s), t) y^{\prime}(s)= \\
& =C \vec{u}(x(s), y(s), t) \cdot \overrightarrow{\mathbf{n}}(x(s), y(s))=0 .
\end{aligned}
$$

Portanto podemos escolher, para cada $t$, uma constante aditiva de modo que $\Psi$ satisfaça:

$$
\Psi(x, y, t)=0 \quad \forall(x, y) \in \partial U, \forall t \in \mathbb{R} .
$$


Além disto, é imediato a partir de (2.34) e (2.36), que $\Psi$ obedece a equação de Poisson:

$$
\Delta \Psi=-\xi
$$

onde $\Delta=\frac{\partial^{2}}{\partial x^{2}}+\frac{\partial^{2}}{\partial y^{2}}$ é o Laplaciano em $\mathbb{R}^{2}$.

Resumindo estes resultado, temos que as equações que determinam completamente o fluxo de um fluido nas hipóteses acima são:

$$
\begin{gathered}
\frac{D \xi}{D t}=\frac{\partial \xi}{\partial t}+(\vec{u} \cdot \nabla) \xi=0, \\
\Delta \Psi=-\xi
\end{gathered}
$$

com

$$
\left.\Psi\right|_{\partial U}=0
$$

$\mathrm{e}$

$$
u_{x}=\frac{\partial \Psi}{\partial y}, \quad u_{y}=-\frac{\partial \Psi}{\partial x}
$$

Observação 2.9 De (2.37) e (2.14) obtemos imediatamente que:

$$
\xi(\varphi(\vec{x}, t), t)=\xi(\vec{x}, 0) \quad \forall t \in \mathbb{R} .
$$

A expressão (2.41), nos diz que a vorticidade é transportada pelas trajetórias sem deformação. Este resultado implica, em particular, que:

\section{Proposição 2.2.3}

$$
\|\xi(\vec{x}, t)\|_{p}=\|\xi(\vec{x}, 0)\|_{p} \quad \forall t \in \mathbb{R}, 1 \leq \mathrm{p} \leq \infty
$$

onde a norma $\|\cdot\|_{p}$ é definida por

$$
\|\xi(\vec{x}, t)\|_{p}=\left(\iint_{U}|\xi(\vec{x}, t)|^{p} d x d y\right)^{1 / p}
$$


Demonstração: Basta aplicar o teorema de mudança de variáveis a $U$, lembrando que $U_{t}=U$ e que o Jacobiano de $\varphi_{t}$ é 1 .

É bem conhecido (ver, por exemplo, [10]) que a solução da equação de Poisson em $U$, com a condição (2.39), pode ser escrita em termos da função de Green neste domínio. É possível, então, determinar explicitamente a função de corrente em termos do campo escalar de vorticidade. Recordemos que se $U \subset \mathbb{R}^{2}$ é um domínio limitado, simplesmente conexo, com fronteira suficientemente suave, a função de Green $G: U \times \bar{U} \rightarrow \mathbb{R}$, com $G(\vec{x}, \vec{y})=0, \forall \vec{x} \in U, \vec{y} \in \partial \bar{U}$, singular nos pontos $(\vec{x}, \vec{x})$, com $\vec{x} \in U$, satisfaz a expressão:

$$
\Delta_{x} G(\vec{x}, \vec{y})=\delta(\vec{x}-\vec{y})
$$

onde $\delta$ é a distribuição delta de Dirac. Embora a função de Green seja na realidade uma distribuição, pode-se demonstrar que $G(\vec{x}, \cdot)$ é infinitamente diferenciável em $U-\{\vec{x}\}$, que $G(\vec{y}, \vec{x})-\frac{1}{2 \pi} \ln \|\vec{x}-\vec{y}\|$ é harmônica em $U$, e que $G(\vec{x}, \vec{y})=G(\vec{y}, \vec{x}), \forall \vec{x}, \vec{y} \in U$, $\vec{x} \neq \vec{y}$.

Observação 2.10 No caso de U não ser limitado, a função de Green ainda pode ser determinada se à condição de fronteira acrescentarmos condições adequadas sobre seu comportamento assintótico.

A função de corrente é explicitamente determinada por:

$$
\Psi(\vec{x})=-\iint_{U} \xi(\vec{z}) G(\vec{x}, \vec{z}) d \vec{z}
$$

Recordemos ainda, que no caso $U=\mathbb{R}^{2}$ a função de Green é dada por:

$$
G(\vec{x}, \vec{y})=\frac{1}{2 \pi} \ln \|\vec{x}-\vec{y}\|
$$

onde $\|\cdot\|$ é a norma euclidiana em $\mathbb{R}^{2}$. 


\subsection{Alguns resultados sobre circulação}

Para motivarmos algumas escolhas no modelo dos vórtices, enunciaremos aqui alguns resultados clássicos sobre circulação. Consideraremos novamente um fluido incompressível, ideal, com densidade constante, confinado numa região $D \subset \mathbb{R}^{3}$.

Definição 2.8 A circulação do campo de velocidade ao redor de $C_{t}=\varphi_{t}(C)$, onde $C$ é uma curva fechada lisa, deslocando-se com o fluido, é definida como a integral de linha

$$
\Gamma_{C_{t}}=\oint_{C_{t}} \vec{u} \cdot d s
$$

Sobre a circulação temos o seguinte teorema clássico:

Teorema 2.3.1 (Kelvin). Se $\mathbf{b}$ é proveniente de um potencial $\Phi$, isto é, $\mathbf{b}=$ grad $\Phi$ e $C$ é qualquer curva fechada, suave, deslocando-se com o fluido, segundo a equação de Euler (2.19), então o fluxo preserva a circulação (2.47), ou seja:

$$
\frac{d}{d t} \Gamma_{C_{t}}=\frac{d}{d t} \oint_{C_{t}} \vec{u} \cdot d s=0
$$

A demostração deste teorema é baseada no teorema de transporte da circulação para curvas:

Teorema 2.3.2 (Transporte de circulação) Seja $\vec{u}$ o campo de velocidade do fluxo $e$ seja $C$ uma curva suave, fechada, em $D$, com $C_{t}=\varphi_{t}(C)$ a curva transportada pelo fluxo. Então:

$$
\frac{d}{d t} \int_{C_{t}} \vec{u}(\vec{x}, t) \cdot d s=\int_{C_{t}} \frac{D \vec{u}}{D t}(\vec{x}, t) \cdot d s
$$

Demostração: Seja $\vec{x}(s)$ uma parametrização da curva $C$, com $0 \leq s \leq 1$. Então $\varphi(\vec{x}(s), t)$, com $0 \leq s \leq 1$ é umaa parametrização de $C_{t}$. Assim, pela definição da integral de linha e da derivada material (2.15) temos que:

$$
\frac{d}{d t} \int_{C_{t}} \vec{u}(\vec{x}, t) \cdot d s=\frac{d}{d t} \int_{0}^{1} \vec{u}(\varphi(\vec{x}(s), t), t) \cdot \frac{\partial}{\partial s} \varphi(\vec{x}(s), t) d s
$$




$$
\begin{aligned}
= & \int_{0}^{1} \frac{D \vec{u}}{D t}(\varphi(\vec{x}(s), t), t) \cdot \frac{\partial}{\partial s} \varphi(\vec{x}(s), t) d s \\
& +\int_{0}^{1} \vec{u}(\varphi(\vec{x}(s), t), t) \cdot \frac{\partial}{\partial s} \frac{\partial}{\partial t} \varphi(\vec{x}(s), t) d s .
\end{aligned}
$$

Como $\frac{\partial \varphi}{\partial t}(\vec{x}(s), t)=\vec{u}(\varphi(\vec{x}(s), t), t)$ e $C_{t}$ é uma curva fechada temos:

$$
\int_{0}^{1} \vec{u}(\varphi(\vec{x}(s), t), t) \cdot \frac{\partial}{\partial s} \frac{\partial}{\partial t} \varphi(\vec{x}(s), t) d s=\frac{1}{2} \int_{0}^{1} \frac{\partial}{\partial s}(\vec{u} \cdot \vec{u})(\varphi(\vec{x}(s), t), t) d s=0 .
$$

Demostração (do teorema de Kelvin): Usando o teorema anterior, a equação de Euler (2.19) e considerando que $C_{t}$ é uma curva fechada temos:

$$
\frac{d}{d t} \int_{C_{t}} \vec{u}(\vec{x}, t) \cdot d s=\int_{C_{t}} \frac{D \vec{u}}{D t}(\vec{x}, t) \cdot d s=-\int_{C_{t}} \operatorname{grad}\left(\frac{p}{\rho}-\Phi\right)(\vec{x}, t) \cdot d s=0 .
$$

Observação 2.11 Notemos que $\Gamma_{C_{t}}$ representa a circulação do campo vetorial $\vec{u}$ ao redor de $C_{t}$; soma as componentes tangenciais do $\vec{u}$ ao redor da curva $C_{t}$. O teorema de Stokes nos dá uma interpretação física do campo de vorticidade $\vec{\Omega}=\nabla \times \vec{u}$ definido em (2.23). De fato, se aplicarmos o teorema de Stokes ao disco $D$ de centro $\vec{x}_{0}$ e radio $\delta$ obtemos

$$
\Gamma_{D}=\int_{\partial D} \vec{u} \cdot d s=\iint_{D} \vec{\Omega} \cdot \hat{\mathbf{n}} d A,
$$

onde $\hat{\mathbf{n}}$ é o vetor unitário normal a $D$. Se denotamos $D=D_{\delta}$ e aplicamos o teorema de valor médio para integrais a (2.50) obtemos

$$
(\vec{\Omega} \cdot \hat{\mathbf{n}})\left(\vec{x}_{0}\right)=\lim _{\delta \rightarrow 0} \frac{1}{A\left(D_{\delta}\right)} \int_{\partial D_{\delta}} \vec{u} \cdot d s,
$$

onde $A\left(D_{\delta}\right)$ é área de $D_{\delta}$. Portanto $\vec{\Omega} \cdot \hat{\mathbf{n}}=(\nabla \times \vec{u}) \cdot \hat{\mathbf{n}}$ é a circulação do campo vetorial $\vec{u}$ por unidade de área sobre uma superfície perpendicular a $\hat{\mathbf{n}}$. A magnitude de $\vec{\Omega} \cdot \hat{\mathbf{n}} e ́$ máxima quando $\hat{\mathbf{n}}=\frac{\vec{\Omega}}{\|\vec{\Omega}\|}$.

Observação 2.12 Consideremos agora uma superfície $V \subset D$, com fronteira suave, fechada C. Pelo teorema de Stokes,

$$
\iint_{V_{t}} \vec{\Omega} \cdot \hat{\mathbf{n}} d A=\int_{C_{t}} \vec{u} \cdot d s .
$$

Assim, como um corolário do teorema de Kelvin (2.3.1), temos que a vorticidade através de uma superfície deslocando-se com o fluido é constante no tempo. 
Finalmente, vamos introduzir as definições de linha de vórtice e tubo de vórtice:

Definição 2.9 Uma linha de vórtice no instante $t$ fixado é uma curva $\vec{x}(s), s \in[0,1]$, em $D$, tangente em cada ponto ao vetor vorticidade neste instante, isto é, $\dot{\vec{x}}(s)$ é paralelo a $\vec{\Omega}(\vec{x}(s), t)$, para todo $s \in[0,1]$. Dada uma superfície $V \subset D$, transversal a $\vec{\Omega}_{t}$ em todos seus pontos, com fronteira fechada e suave, a superfície formada pelas linhas de vórtice, no instante $t$, passando pelos pontos de $\delta V$ é chamado um tubo de vórtice no instante $t$.

Observe que se $C$ é uma linha de vórtice em $t=0$, então $C_{t}=\varphi_{t}(C)$ é uma linha de vórtice no instante $t$, e que se $S$ é um tubo de vórtice em $t=0$ então $S_{t}=\varphi_{t}(S)$ é um tubo de vórtice no instante $t$. Pode-se demonstrar que:

Teorema 2.3.3 (Helmholtz). Sob as mesmas hipótese do teorema de Kelvin (2.3.1), se $C_{1}$ e $C_{2}$ são duas curvas fechadas suaves que circundam o mesmo tubo vórtice, então:

$$
\int_{C_{1}} \vec{u} \cdot d s=\int_{C_{2}} \vec{u} \cdot d s
$$

Demostração: basta aplicar os teoremas do divergente e de Stokes às superfícies definidas pelo tubo de vórtice e pelas curvas $C_{1}$ e $C_{2}$ ( ver [7]).

Este valor comum é chamado intensidade do tubo vórtice e, pelo resultado de Kelvin, é constante no tempo. 


\section{Capítulo 3}

\section{O modelo dos vórtices sem massa}

Neste capítulo apresentamos o modelo dos vórtices sem massa, relacionado com duas situações físicas importantes. A primeira, é a de um fluido em que a vorticidade está concentrada num número finito de pontos do plano. A segunda, é a de um sistema de "cargas bidimensionais", as quais podem ser vistas como a interseção de uma coluna infinita de partículas carregadas com um plano ortogonal.

Na seção 3.1, obteremos heuristicamente, a partir das equações do capítulo 2 , as equações diferenciais ordinárias de Helmholtz e Kirchhoff, que governam o movimento de $\mathrm{N}$ vórtices puntuais. Na seção 3.2 , introduziremos as definições básicas em sistemas Hamiltonianos e mostraremos que o modelo dos vórtices é um sistema Hamiltoniano. Ainda nesta seção, introduziremos o conceito de integral primeira e o de integrabilidade segundo Liouville. Exibiremos duas integrais primeiras independentes em involução, além da energia, o que implica, em particular, que sistemas se dois ou três vórtices são sempre integráveis. Na seção 3.3, usando analogia eletromagnética, mostramos que estas equações também determinam o comportamento de cargas bidimensionais sem massa, isto é, as equações do modelo dos vórtices puntuais são os limites formais, quando as massas tendem a zero, das equações do movimento de $\mathrm{N}$ cargas bidimensionais.

Na seção 3.4, analisaremos, primeiramente, o sistema de dois vórtices sem massa. A 
análise será feita separadamente no caso da soma das intensidades ser ou não nula. Em ambos casos, a dinâmica dos vórtices não só é Liouville integrável, como também é simples determiná-la explicitamente. A seguir, consideraremos o exemplo de três vórtices, com intensidades $(1,1,1)$. Embora este sistema continue sendo Liouville integrável, neste caso já não será possível exibir explicitamente todas as suas trajetórias. Entretanto, ainda é possível exibir explicitamente uma trajetória que é uma conexão tipo sela para este sistema e órbitas numa vizinhança desta conexão. Finalmente, é analisado o sistema de quatro vórtices com intensidades $(1,1,1, \varepsilon)$ como perturbação do sistema de três vórtices com intensidades $(1,1,1)$ (ver [5]). Para $\varepsilon$ não negativo e suficientemente pequeno o problema perturbado é reduzido, numa vizinhança da conexão de sela admitida pelo problema não perturbado, a um sistema de equações diferenciais no plano, o qual não é mais autônomo, mas depende periodicamente do tempo. Assim, mediante um resultado devido a Melnikov (ver [8] e [15]), é possível mostrar que o problema perturbado admite interseções transversais da variedade estável e da variedade instável dos pontos hiperbólicos, tipo sela, e consequentemente comportamento caótico. Este resultado demonstra em particular a não integrabilidade de um sistema com quatro vórtices. Ziglin, em [29], já havia demonstrado este resultado por outro método, que descreveremos no final do capítulo.

\subsection{Vórtices puntuais: modelo de Helmholtz-Kirchhoff}

Para simplificar as equações do movimento da vorticidade, define-se um modelo que reduz um problema de equações diferenciais parciais a um problema de equações diferenciais ordinárias, com um número finito de graus de liberdade. Este modelo é definido supondo que a vorticidade está concentrada em um número finito de pontos $\vec{x}_{1}, \vec{x}_{2}, \cdots, \vec{x}_{N}$ num domínio $U \subset \mathbb{R}^{2}$, ou seja, a vorticidade é uma distribuição com suporte nestes pontos. Cada um destes pontos, onde a vorticidade é singular, é chamado de vórtice. Pelo teorema de Kelvin, o valor da circulação do campo de velocidade ao redor de um deles é constante, e será chamado de intensidade do vórtice. Além do fato das equações discretizadas repre- 
sentarem situações físicas concretas, um interesse adicional nestas equações discretizadas surge do fato que elas representam um sistema Hamiltoniano.

Seja $U \subset \mathbb{R}^{2}$ um domínio simplesmente conexo, onde esteja definida uma função de Green G. Vamos considerar o movimento do fluido no caso particular descrito na seção 2.3, ou seja, este movimento é determinado pelas equações (2.37) a (2.40). Heuristicamente, se disminuirmos a região em que a vorticidade está concentrada, mantendo no entanto sua intensidade, podemos pensar em soluções no sentido de distribuição. Assim, admitindo as equações válidas também no caso da vorticidade ser uma distribuição, suporemos o campo escalar da vorticidade na forma

$$
\xi(\vec{z})=\sum_{j=1}^{N} \xi_{j}(\vec{z})=\sum_{j=1}^{N} \Gamma_{j} \delta\left(\vec{z}-\vec{x}_{j}\right),
$$

onde $\Gamma_{j}$ é a intensidade do $j$-ésimo vórtice e $\delta$ é o delta de Dirac, e de (2.38) obteremos que a função de corrente será dada por:

$$
\Psi(\vec{x}, t)=\Psi_{0}(\vec{x})-\sum_{j=1}^{N} \Gamma_{j} G\left(\vec{x}, \vec{x}_{j}(t)\right)=\Psi_{0}(\vec{x})-\sum_{j=1}^{N} \Psi_{j}(\vec{x}, t),
$$

onde $\Psi_{0}$ é uma função harmônica em $U$ (função de corrente devida a fatores externos).

Quando os $\mathrm{N}$ vórtices $\vec{x}_{1}, \vec{x}_{2}, \cdots, \vec{x}_{N}$ em $U$ estão em movimento eles induzem em $\vec{x}$ o campo de velocidade

$$
\vec{u}(\vec{x}, t)=\sum_{j=1}^{N} \vec{u}_{j}(\vec{x}, t)
$$

onde

$$
\vec{u}_{j}(\vec{x}, t)=\left(\frac{\partial \Psi_{j}}{\partial y}\left(\vec{x}, \vec{x}_{j}(t)\right),-\frac{\partial \Psi_{j}}{\partial x}\left(\vec{x}, \vec{x}_{j}(t)\right)\right)
$$

é o campo de velocidade em $\vec{x}=(x, y)$, induzido pelo $j$-ésimo vórtice. Note que este campo de velocidade é singular nos vórtices, e para determinarmos suas trajetórias precisamos fazer alguma hipótese regularizadora. Se assumirmos que cada vórtice não se movimenta por ação de seu campo, mas sim por ação do campo de velocidade gerado pelos outros vórtices, temos o conhecido modelo dos vórtices puntais de Helmholtz e Kirchhoff (ver[11]). 
Neste modelo, as equações de movimento do $j$-ésimo vórtice são:

$$
\begin{aligned}
& \dot{x_{j}}=\frac{\partial \Psi_{j}^{R}}{\partial y_{j}}\left(\vec{x}_{1}, \ldots, \vec{x}_{N}\right), \quad j=1,2, \cdots, N \\
& \dot{y}_{j}=-\frac{\partial \Psi_{j}^{R}}{\partial x_{j}}\left(\vec{x}_{1}, \ldots, \vec{x}_{N}\right) \text {, }
\end{aligned}
$$

onde

$$
\Psi_{j}^{R}\left(x_{1}, \ldots, x_{N}\right)=\Psi_{0}\left(\vec{x}_{j}\right)-\sum_{\substack{i=1 \\ i \neq j}}^{N} \Gamma_{i} G\left(\vec{x}_{j}, \vec{x}_{i}\right)
$$

é a função de corrente regularizada e $\vec{x}_{j}=\left(x_{j}, y_{j}\right)$, para todo $j=1, \ldots, N$.

\subsection{O modelo dos vórtices sem massa como um mo- delo Hamiltoniano}

É notável o fato que a dinâmica dos vórtices pode ser descrita por um sistema Hamiltoniano, como mostra o próximo teorema, que é um resultado devido a Lin. Lembremos, primeiramente, as principais definições e resultados sobre sistemas Hamiltonianos.

Definição 3.1 Seja $M=M^{2 n}$ uma variedade diferenciável de dimensão par. Uma variedade simplética é um par $(M, w)$, onde w é uma forma simplética, isto é, uma 2-forma diferenciável, não degenerada e fechada, definida sobre $M$.

Um dos fatos que tornam as variedades simpléticas espaços adequados ao estudo de sistemas dinâmicos é que elas admitem coordenadas especiais, chamadas de coordenadas canônicas ou coordenadas de Darboux, descritas na proposição:

Proposição 3.2.1 Teorema de Darboux Seja $(M, w)$ uma variedade simplética. Então, para todo $x \in M$, existe uma vizinhança $U$ de $x$ e um sistema de coordenadas $\left(p_{1}, \cdots, p_{n}\right.$, $\left.q_{1}, \cdots, q_{n}\right)$ onde $w$ tem a forma $w=\sum_{i=1}^{n} d p_{i} \wedge d q_{i}$. 
As variáveis $p_{i}$ são chamadas variáveis tipo momento, e as variáveis $q_{i}$ são chamadas variáveis tipo posição. Quando estudamos um sistema dinâmico, procuramos escolher um sistema de coordenadas que simplifique o problema tratado. No caso de variedades simpléticas e sistemas Hamiltonianos, estaremos sempre interessados em coordenadas de Darboux, o que justifica a definição:

Definição 3.2 Uma transformação canônica é uma mudança de coordenadas que leve um sistema de coordenadas de Darboux em outro sistema de coordenadas de Darboux.

A forma simplética $w$ nos permite associar a cada função diferenciável um campo de vetores:

Definição 3.3 Seja $(M, w)$ uma variedade simplética e seja $F: M \rightarrow \mathbb{R}$, uma $C^{\infty}$ função. O campo vetorial Hamiltoniano $X_{F}$ é definido por:

$$
w(x)\left(\eta, X_{F}(x)\right)=d F(x)(\eta) \quad \forall \eta \in T_{x} M, \forall x \in M
$$

Notemos que $X_{F}$ está bem definido, já que $w$ é não degenerada. Outro conceito importante na teoria dos sistemas Hamiltonianos é o colchete de Poisson:

Definição 3.4 Numa variedade simplética, o colchete de Poisson $\{\cdot, \cdot\}: C^{\infty}(M) \times$ $C^{\infty}(M) \rightarrow C^{\infty}(M)$ é definido por:

$$
\{F, G\}=w\left(X_{G}, X_{F}\right), \forall F, G \in C^{\infty}(M)
$$

Observação 3.1 O fato de w ser uma forma simplética implica que o colchete de Poisson é bilinear, antissimétrico, satisfaz a identidade de Jacobi, e que $\{f, \cdot\}$ é uma derivação em $C^{\infty}(M)$.

Podemos, finalmente, definir: 
Definição 3.5 Suponha que exista uma função $H \in C^{\infty}(M)$ tal que as trajetórias de um sistema dinâmico em $M$ sejam as curvas integrais de $X_{H}$, isto é, se $\gamma(t)$ é uma trajetória em $M, \dot{\gamma}(t)=X_{H}(\gamma(t))$. Neste caso, dizemos que $(M, w, H)$ é um sistema Hamiltoniano, a função $H$ é a função Hamiltoniana e n é o número de graus de liberdade do sistema.

Observação 3.2 Em coordenadas de Darboux, as equações de movimento de um sistema Hamiltoniano, com Hamiltoniana $H$, se escrevem como:

$$
\dot{q}_{i}=\frac{\partial H}{\partial p_{i}} \quad, \quad \dot{p}_{i}=-\frac{\partial H}{\partial q_{i}} .
$$

Voltemos agora ao estudo do modelo dos vórtices. É imediato que as equações (3.5) são equivalentes a:

Teorema 3.2.1 (C.C.Lin) $O$ movimento do sistema de $N$ vórtices no domínio $U$, é governado pelo sistema Hamiltoniano $\left(U^{N}, w, W\right)$, onde

$$
\begin{aligned}
W\left(\vec{x}_{1}, \vec{x}_{2}, \cdots \vec{x}_{N}\right) & =\sum_{j=1}^{N} \Gamma_{j} \Psi_{o}\left(\vec{x}_{j}\right)-\frac{1}{2} \sum_{\substack{i, j=1 \\
i \neq j}}^{N} \Gamma_{i} \Gamma_{j} G\left(\vec{x}_{i}, \vec{x}_{j}\right), \\
w & =\sum_{j=1}^{N} \Gamma_{j} d y_{j} \wedge d x_{j} .
\end{aligned}
$$

Observação 3.3 Se F é uma função em $C^{\infty}(U)$, então o campo Hamiltoniano correspondente é dado por:

$$
X_{F}=\sum_{j=1}^{N} \frac{1}{\Gamma_{j}}\left(\frac{\partial F}{\partial y_{j}} \frac{\partial}{\partial x_{j}}-\frac{\partial F}{\partial x_{j}} \frac{\partial}{\partial y_{j}}\right) .
$$

Se $G$ é outra função em $C^{\infty}(U)$, então o colchete de Poisson é dado por:

$$
\{F, G\}=\sum_{j=1}^{N} \frac{\partial F}{\partial x_{j}} \frac{\partial G}{\partial y_{j}}-\frac{\partial G}{\partial x_{j}} \frac{\partial F}{\partial y_{j}}
$$

Observação 3.4 Lin chama a função hamiltoniana $W$ de função de Kirchhoff-Routh e seu teorema implica que as equações do movimento dos vórtices num domínio U são dadas 
por:

$$
\begin{aligned}
\Gamma_{j} \dot{x}_{j} & =\frac{\partial W}{\partial y_{j}} \\
\Gamma_{j} \dot{y}_{j} & =-\frac{\partial W}{\partial x_{j}} .
\end{aligned}
$$

Nesta dissertação consideraremos apenas o caso em que $U=\mathbb{R}^{2}$ e em que o fluido, na ausência dos vórtices, está em repouso, o que implica $\Psi_{0}=0$. Usando que em $\mathbb{R}^{2}$ a função de Green é dada por (2.46), temos o seguinte corolário:

Corolario 3.2.1 O movimento do sistema de $N$ vórtices no domínio $D=\mathbb{R}^{2}$ é governado pelo sistema Hamiltoniano $\left(\mathbb{R}^{2 \mathrm{~N}}, w, W\right)$, com função Hamiltoniana $W$ definida por

$$
W=-\frac{1}{4 \pi} \sum_{\substack{i, j=1 \\ i \neq j}}^{N} \Gamma_{i} \Gamma_{j} \ln \left\|\vec{x}_{i}-\vec{x}_{j}\right\|,
$$

e forma simplética

$$
w=\sum_{j=1}^{N} \Gamma_{j} d y_{j} \wedge d x_{j}
$$

Demonstração: De fato, pela definição de sistema Hamiltoniano, as equações que descrevem o movimento do sistema de $N$ vórtices são :

$$
\begin{aligned}
\Gamma_{j} \dot{x}_{j} & =\frac{\partial W}{\partial y_{j}} \\
\Gamma_{j} \dot{y}_{j} & =-\frac{\partial W}{\partial x_{j}} .
\end{aligned}
$$

Dividindo as equações (3.16) por $\Gamma_{j}$, com $W$ definida em (3.14), obtemos exatamente as equações de Helmholtz-Kirchhoff do movimento do $j$-ésimo vórtice, definidas em (3.5).

Sistemas Hamiltonianos geralmente são classificados em integráveis ou não integráveis. Se o sistema é integrável, sua dinâmica é essencialmente quase periódica e então 
pode-se dizer que o sistema é bem comportado. E esta é a única situação onde obtém-se uma descrição global mais ou menos completa da dinâmica do sistema. Devido a isto, é essencial decidir se um dado sistema é ou não integrável.

Para caracterizarmos um sistema integrável temos um teorema clássico, devido a Liouville. Esta caracterização será feita em termos de integrais primeiras, cuja definição damos a seguir.

Definição 3.6 Seja $(M, w)$ uma variedade simplética e seja $X_{H}$ o campo vetorial Hamiltoniano correspondente à função hamiltoniana $H$. Uma função $F: M \rightarrow \mathbb{R}$ de classe $C^{\infty}$ é uma integral primeira do campo $X_{H}$ se é constante sobre as trajetórias de $X_{H}$, isto é, se $d F\left(X_{H}\right)=0$.

Definição 3.7 Seja $(M, w)$ uma variedade simplética. Duas funções $F, G: M \rightarrow \mathbb{R}$ de classe $C^{\infty}$ estão em involução se $\{F, G\}=0$.

Observação 3.5 Note que $F$ é integral primeira de $X_{H}$ se, e somente se,

$$
d F\left(X_{H}\right)=w\left(X_{H}, X_{F}\right)=\{F, H\}=0
$$

ou seja, se $F$ e $H$ estão em involução.

Podemos então enunciar:

Teorema 3.2.2 (Liouville) Seja $\left(M^{2 n}, w, H\right)$ um sistema Hamiltoniano e suponha que são dadas $F_{1}, \cdots, F_{n}$ integrais primeiras em involução. Seja $M_{\lambda}=\left\{x \in M\right.$ tal que $F_{i}(x)$ $\left.=\lambda_{i}, i=1 \cdots, n\right\}$, com $\lambda=\left(\lambda_{1} \cdots \lambda_{n}\right) \in \mathbb{R}^{\mathrm{n}}$, e suponha que $\left(d F_{1}(x), \cdots, d F_{n}(x)\right)$ são linearmente independente, para cada $x \in M_{\lambda}$. Então

1. $M_{\lambda}$ é uma subvariedade de dimensão $n$ de $M$, invariante pelo fluxo dos campos de vetores $X_{F_{1}}, \cdots, X_{F_{n}}$. Além disso $w\left(X_{F_{i}}, X_{F_{j}}\right)=0$. 
2. Suponha que $X_{F_{i}}, i=1, \cdots, n$ são completos, isto é, os fluxos gerados por estes campos são definidos para todo $t \in \mathbb{R}$. Então $M_{\lambda}$ é difeomorfa a $\mathbb{R}^{\mathrm{k}} \times T^{n-k}$, para algum inteiro $k$, onde $0 \leq k \leq n$. Em particular, se $M_{\lambda}$ é compacta então $M_{\lambda}$ é difeomorfa ao toro $T^{n}$ (toro $n$-dimensional).

3. Sob as mesmas hipoteses de 2), seja $M_{\lambda}$ compacta e $F_{1}=H$. Então o fluxo do campo vetorial $X_{H}$ define sobre $M_{\lambda}$ movimentos quase-periódicos $\varphi(t)$ dados nas coordenadas angulares $\varphi_{1}, \ldots, \varphi_{n}$ por

$$
\frac{d \varphi}{d t}=\nu, \quad \nu=\nu(\lambda)
$$

4. Sob as mesmas hipôteses de 3), é possivel encontrar funções $I_{1}, \ldots, I_{n}$ que dependem só de $F_{1}, \cdots, F_{n}$, chamadas coordenadas ação, tal que $\left(I_{1}, \ldots, I_{n}, \varphi_{1}, \ldots, \varphi_{n}\right)$ são coordenadas canônicas numa vizinhança de $M_{\lambda}$, e então as trajetórias de $X_{H}$ são dadas por:

$$
\frac{d I}{d t}=0, \quad \frac{d \varphi}{d t}=\nu(I)
$$

onde $H=H(I)$ e $\nu(I)=\frac{\partial H}{\partial I}(I)$.

Definição 3.8 Um sistema Hamiltoniano satisfazendo as hipóteses do teorema 3.2.2 é chamado integrável, no sentido de Liouville.

O teorema anterior nos diz ainda que dado um sistema mecânico com espaço de fase de dimensão $2 n$, admitindo $k \leq n$ integrais primeiras em involução e independentes (isto é, suas diferenciais são linearmente independentes), pode-se reduzir a dimensão do espaço de fase a $2(n-k)$. Em particular, se $n=k$, as equações do movimento podem ser explicitamente integradas. De fato, as trajetórias são "linhas retas"sobre cilindros ou toro. Se o movimento toma lugar no toro, a integração explícita das equações do movimento, são determinadas ao se encontrar as variáveis ângulo-ação (ver [2] ou [3]).

Assim, para a caracterização do sistema dos vórtices sem massa, precisamos conhecer suas integrais primeiras. No caso dos vórtices em $\mathbb{R}^{2}$, temos, considerando as simetrias 
da função Hamiltoniana $W$, que o sistema $\left(\mathbb{R}^{2 \mathrm{~N}}, w, W\right)$ tem as três integrais primeiras abaixo, além da energia $W$ :

$$
\begin{aligned}
& \Pi_{x}=\sum_{j=1}^{N} \Gamma_{j} y_{j}, \\
& \Pi_{y}=-\sum_{j=1}^{N} \Gamma_{j} x_{j}, \\
& J==-\frac{1}{2} \sum_{j=1}^{N} \Gamma_{j}\left\|\vec{x}_{j}\right\|^{2} .
\end{aligned}
$$

A partir da equação (3.12), é fácil mostrar que seus colchetes de Poisson são dados por:

$$
\begin{aligned}
\left\{\Pi_{x}, \Pi_{y}\right\} & =-\sum_{j=1}^{N} \Gamma_{j}, \\
\left\{\Pi_{x}, J\right\} & =\Pi_{y}, \\
\left\{\Pi_{y}, J\right\} & =\Pi_{x} .
\end{aligned}
$$

Como estas integrais primeiras não estão em involução, define-se

$$
\Pi^{2}=\Pi_{x}^{2}+\Pi_{y}^{2}
$$

que satisfaz

$$
\left\{\Pi^{2}, J\right\}=0 \text {. }
$$

Dos resultados anteriores temos a seguinte proposição:

Proposição 3.2.3 As funções $\Pi^{2}$ e $J$ (além da energia $W$ ) são integrais primeiras independentes e em involução do sistema Hamiltoniano $\left(\mathbb{R}^{2 \mathrm{~N}}, w, W\right)$, com função Hamiltoniana $W$ e forma simplética $w$ definida em (3.14) e (3.15), respectivamente.

A partir da proposição anterior, pode-se garantir que o sistema de dois e três vórtices sempre é integrável, no sentido de Liouville. Entretanto, já no caso de quatro vórtices 
isto nem sempre é verdade. Veremos na seção 3.4 um exemplo de um sistema de quatro vórtices que não é integrável, o que mostra que não existe, no caso geral, três integrais primeiras (além de $W$ ) independentes em involução.

\subsection{Partículas carregadas num campo magnético e sua relação com o modelo dos vórtices}

Nesta seção apresentaremos o modelo para um sistema de $N$ "cargas bidimensionais", as quais podem ser vistas como a interseção de $N$ colunas infinitas de partículas carregadas com um plano ortogonal (plano $x y$ ), sujeitas a um campo magnético constante paralelo às colunas (na direção do eixo $z$ ), sendo o potencial elétrico gerado por este sistema de cargas logarítmico. Apresentaremos este modelo tanto em sua forma Lagrangeana quanto em sua forma Hamiltoniana. A partir das equações Lagrangeanas deste sistema de $N$ partículas carregadas, fazendo as massas das cargas tenderem a zero, obtemos, formalmente, as equações dos vórtices puntuais de Helmoltz-Kirchhoff, definidos em (3.5).

Se supusermos que a velocidade e aceleração das cargas não são muito grandes, de forma que possamos desprezar os efeitos de relatividade e de radiação, temos que a função Lagrangeana $L: T\left(\mathbb{R}^{2 \mathrm{~N}}\right) \approx \mathbb{R}^{4 \mathrm{~N}} \rightarrow \mathbb{R}$, associada ao sistema de partículas carregadas descrito acima é definida por:

$$
L\left(\vec{x}_{1}, \cdots, \vec{x}_{N}, \dot{\vec{x}}_{1}, \cdots, \dot{\vec{x}}_{N}\right)=\sum_{j=1}^{N} \frac{m_{j}}{2}\left\|\dot{\vec{x}}_{j}\right\|^{2}-\mathrm{U}\left(\vec{x}_{1}, \cdots, \vec{x}_{N}, \dot{\vec{x}}_{1}, \cdots, \dot{\vec{x}}_{N}\right),
$$

com

$$
\begin{aligned}
\mathrm{U}\left(\vec{x}_{1}, \cdots, \vec{x}_{N}, \dot{\vec{x}}_{1}, \cdots, \dot{\vec{x}}_{N}\right)=\Phi\left(\vec{x}_{1}, \cdots, \vec{x}_{N}\right)-\sum_{j=1}^{N} \frac{Q_{j}}{c} \dot{\vec{x}}_{j} \cdot \mathbf{A}_{j}\left(\vec{x}_{j}\right), \\
\mathbf{A}_{j}=\frac{B}{2}\left(\mathbf{e}_{3} \times \vec{x}_{j}\right) \quad, \quad \Phi\left(\vec{x}_{1}, \cdots, \vec{x}_{N}\right)=-\frac{1}{4 \pi} \sum_{\substack{i, j=1 \\
i \neq j}}^{N} Q_{i} Q_{j} \ln \left\|\vec{x}_{i}-\vec{x}_{j}\right\|,
\end{aligned}
$$

onde $Q_{j}$ e $m_{j}$ são, respectivamente, a intensidade de carga e a massa da j-ésima partícula 
carregada, e é o versor na direção do eixo $z, \mathbf{B}=B \mathbf{e}_{3}$ é o campo magnético uniforme e $c$ é a velocidade da luz.

As equações de Euler-Lagrange são :

$$
\frac{d}{d t}\left(\frac{\partial L}{\partial \dot{x}_{j_{i}}}\right)-\frac{\partial L}{\partial x_{j_{i}}}=0 \quad i=1,2 . \quad j=1,2 . \cdots, N .
$$

onde $\vec{x}_{j}=\left(x_{j_{1}}, x_{j_{2}}\right)=\left(x_{j}, y_{j}\right)$.

A correspondente transformação de Legendre, $F L$, associada a $L$ é a aplicação

$$
F L: T\left(\mathbb{R}^{2 \mathrm{~N}}\right) \rightarrow T^{*}\left(\mathbb{R}^{2 \mathrm{~N}}\right),
$$

que associa a cada vetor $\left(\vec{x}_{j}, \dot{\vec{x}}_{j}\right)$ no plano tagente $T\left(\mathbb{R}^{2 \mathrm{~N}}\right)$, de $\mathbb{R}^{2 \mathrm{~N}}$, o vetor $\left(\vec{x}_{j}, \vec{p}_{j}\right)=$ $F L\left(\vec{x}_{j}, \dot{\vec{x}}_{j}\right)$ no plano cotangente $T^{*}\left(\mathbb{R}^{2 \mathrm{~N}}\right)$, de $\mathbb{R}^{2 \mathrm{~N}}$, onde

$$
\vec{p}_{j}=\left(\frac{\partial L}{\partial \dot{x}_{j}}, \frac{\partial L}{\partial \dot{y}_{j}}\right)=m_{j} \dot{\vec{x}}_{j}+\frac{Q_{j} B}{2 c}\left(\mathbf{e}_{3} \times \vec{x}_{j}\right) .
$$

Como $m_{j}>0$, a transformação de Legendre é uma bijeção entre $T\left(\mathbb{R}^{2 \mathrm{~N}}\right)$ e $T^{*}\left(\mathbb{R}^{2 \mathrm{~N}}\right)$. Portanto, temos que ao sistema Lagrangeano está associado um sistema Hamiltoniano, com função Hamiltoniana $H: T^{*}\left(\mathbb{R}^{2 \mathrm{~N}}\right) \rightarrow \mathbb{R}$, definida por $H=E \circ[F L]^{-1}$, onde $E: T\left(\mathbb{R}^{2 N}\right) \rightarrow \mathbb{R}$ é a energia de $L$ (ver [2]), definida por

$$
E=\sum_{j=1}^{N} \vec{p}_{j} \cdot \dot{\vec{x}}_{j}-L
$$

Notemos que, se é utilizada a transformação de Legendre, as coordenadas $\left(\vec{x}_{j}, \vec{p}_{j}\right)=$ $\left(\left(x_{j}, y_{j}\right),\left(p_{x_{j}}, p_{y_{j}}\right)\right) \in T^{*}\left(\mathbb{R}^{2 \mathrm{~N}}\right)$ são automaticamente canônicas. Portanto, a forma simplética sobre $T^{*}\left(\mathbb{R}^{2 \mathrm{~N}}\right)$, é dada por:

$$
w=\sum_{j=1}^{N} d p_{x j} \wedge d x_{j}+d p_{y_{j}} \wedge d y_{j}
$$

A partir de (3.28), (3.24) e (3.29) temos a função Hamiltoniana $H=E \circ(F L)^{-1}$ na forma:

$$
H\left(\vec{x}_{j}, \vec{p}_{j}\right)=\frac{1}{2} \sum_{j=1}^{N} \frac{1}{m_{j}}\left\|\vec{p}_{j}-\frac{Q_{j} B}{2 c}\left(\mathrm{e} \times \vec{x}_{j}\right)\right\|^{2}+\Phi\left(\vec{x}_{1}, \cdots, \vec{x}_{N}\right)
$$


Portanto, o movimento do sistema de $N$ "cargas bidimensionais" é governado pelo sistema Hamiltoniano $\left(\mathbb{R}^{4 \mathrm{~N}}, w, H\right)$, com função Hamiltoniana $H$ definida em $(3.31)$ e forma simplética $w$ definida em (3.30), cujas equações de movimento são:

$$
\begin{aligned}
\dot{x}_{j_{i}} & =\frac{\partial H}{\partial p_{j_{i}}}, \\
\dot{p}_{j_{i}} & =-\frac{\partial H}{\partial x_{j_{i}}}, \quad i=1,2, \quad j=1, \cdots, N,
\end{aligned}
$$

onde $\vec{p}_{j}=\left(p_{j_{1}}, p_{j_{2}}\right)=\left(p_{x_{j}}, p_{y_{j}}\right)$.

Veremos agora a relação entre o modelo das "cargas bidimensionais"e o modelo dos vórtices sem massa em $\mathbb{R}^{2}$. Das equações de Euler e Lagrange (3.26) temos:

$$
\frac{d}{d t}\left(m_{j} \dot{\vec{x}}_{j_{i}}-\frac{\partial \mathbf{U}}{\partial \dot{\vec{x}}_{j_{i}}}\right)+\frac{\partial \mathbf{U}}{\partial \vec{x}_{j_{i}}}=0, \quad i=1,2, \quad j=1,2, \cdots, N,
$$

isto é,

$$
\begin{aligned}
m_{j} \ddot{x}_{j_{i}} & =\frac{d}{d t}\left(\frac{\partial \mathbf{U}}{\partial \dot{x}_{j_{i}}}\right)-\frac{\partial \mathbf{U}}{\partial x_{j_{i}}} \\
& =\frac{Q_{j} B}{c}\left(\dot{\vec{x}}_{j} \times \mathbf{e}_{3}\right)_{i}-\frac{\partial \Phi}{\partial x_{j_{i}}} \quad i=1,2 . \quad j=1,2, \cdots, N .
\end{aligned}
$$

Fazendo $m_{j}=0$ na expressão (3.33), obtemos

$$
\begin{aligned}
\frac{Q_{j} B}{c} \dot{x}_{j} & =-\frac{\partial \Phi}{\partial y_{j}} \\
\frac{Q_{j} B}{c} \dot{y}_{j} & =\frac{\partial \Phi}{\partial x_{j}}
\end{aligned}
$$

Reescalonando o tempo por $t=\frac{B t^{\prime}}{c}$ e identificando $Q_{j} \leftrightarrow \Gamma_{j}, \Phi \leftrightarrow W$ temos exatamente as equações (3.16) do movimento dos vórtices puntuais.

Podemos concluir, então que as equações (3.16) do modelo dos vórtices puntuais de Helmholtz e Kirchhoff são os limites formais, quando $m_{j} \rightarrow 0$ nas equações de movimento do sistema de $N$ partículas bidimensionais carregadas, num campo magnético uniforme. Tanto no caso de cargas com massa quanto no de cargas sem massa, a dinâmica do sistema 
é Hamiltoniana, embora no caso sem massa a transformação de Legendre seja singular. Notemos ainda que quando $m_{j} \rightarrow 0$ os momentos não desaparecem. Há ausência de momentos "mecânicos", mas há uma constribução eletromagnética para os momentos, dada por $\vec{p}_{j}=Q_{j} B / 2 c\left(\mathbf{e}_{3} \times \vec{x}_{j}\right)$. Assim, algumas das coordenadas funcionam como momento $(y)$, enquanto que as outras funcionam como posição $(x)$. Não se trata, portanto, de um sistema mecânico no sentido habitual, em que a energia cinética é quadrática nos momentos e a Hamiltoniana é a energia cinética mais mais um termo potencial.

Os resultados desta seção sugerem que é razoável pensar em sistema de vórtices com massa, e que um bom candidato para as equações de movimento neste caso seriam as equações (3.33). 


\subsection{Exemplos}

\subsubsection{Exemplo1: dois vórtices sem massa}

Neste caso, a função Hamiltoniana associada ao sistema $\left(\mathbb{R}^{4}, \mathrm{w}, \mathrm{H}\right)$ de dois vórtices sem massa, de acordo com (3.14), é dada por:

$$
H\left(\vec{x}_{1}, \vec{x}_{2}\right)=-\frac{\Gamma_{1} \Gamma_{2}}{4 \pi} \ln \left[\left(x_{1}-x_{2}\right)^{2}+\left(y_{1}-y_{2}\right)^{2}\right]
$$

onde $\vec{x}_{i}=\left(x_{i}, y_{i}\right), \mathrm{i}=1,2$, e a forma simplética $w$ é dada por:

$$
w=\Gamma_{1} d y_{1} \wedge d x_{1}+\Gamma_{2} d y_{2} \wedge d x_{2} .
$$

De (3.16), é imediato que as equações de movimento são dadas por:

$$
\begin{aligned}
& \Gamma_{1} \dot{x}_{1}=-\frac{\Gamma_{1} \Gamma_{2}}{2 \pi} \frac{\left(y_{1}-y_{2}\right)}{r_{12}^{2}}, \\
& \Gamma_{2} \dot{x}_{2}=\frac{\Gamma_{1} \Gamma_{2}}{2 \pi} \frac{\left(y_{1}-y_{2}\right)}{r_{12}^{2}}, \\
& \Gamma_{1} \dot{y}_{1}=\frac{\Gamma_{1} \Gamma_{2}}{2 \pi} \frac{\left(x_{1}-x_{2}\right)}{r_{12}^{2}}, \\
& \Gamma_{2} \dot{y}_{2}=-\frac{\Gamma_{1} \Gamma_{2}}{2 \pi} \frac{\left(x_{1}-x_{2}\right)}{r_{12}^{2}},
\end{aligned}
$$

onde $r_{12}=\left\|\vec{x}_{2}-\vec{x}_{1}\right\|$.

De acordo com (3.20), este sistema tem as seguintes integrais primeiras:

$$
\begin{aligned}
\Pi_{x} & =\Gamma_{1} y_{1}+\Gamma_{2} y_{2}, \\
\Pi_{y} & =-\Gamma_{1} x_{1}-\Gamma_{2} x_{2}, \\
J & =-\frac{\Gamma_{1}}{2}\left\|\vec{x}_{1}\right\|^{2}-\frac{\Gamma_{2}}{2}\left\|\vec{x}_{2}\right\|^{2} .
\end{aligned}
$$

De (3.37), obtemos o sistema linear:

$$
\begin{aligned}
& \dot{x}_{1}-\dot{x}_{2}=-\frac{\Gamma_{1}+\Gamma_{2}}{2 \pi} \frac{\left(y_{1}-y_{2}\right)}{r_{12}^{2}}, \\
& \dot{y}_{1}-\dot{y}_{2}=-\frac{\Gamma_{1}+\Gamma_{2}}{2 \pi} \frac{\left(x_{1}-x_{2}\right)}{r_{12}^{2}} .
\end{aligned}
$$


Note que, como a Hamiltoniana é uma integral primeira, $r_{12}$ é constante ao longo do movimento. Logo, a solução deste sistema é dada por:

$$
\begin{gathered}
x_{1}(t)-x_{2}(t)=\left(x_{1}(0)-x_{2}(0)\right) \cos \Omega t+\left(y_{1}(0)-y_{2}(0)\right) \sin \Omega t, \\
y_{1}(t)-y_{2}(t)=-\left(x_{1}(0)-x_{2}(0)\right) \sin \Omega t+\left(y_{1}(0)-y_{2}(0)\right) \cos \Omega t,
\end{gathered}
$$

onde

$$
\Omega=\frac{\Gamma_{1}+\Gamma_{2}}{2 \pi r_{12}^{2}}
$$

Primeiro caso: $\Gamma_{1}+\Gamma_{2} \neq 0$

Vamos tomar como condições iniciais os pontos:

$$
\begin{array}{ll}
x_{1}(0)=1, & y_{1}(0)=0, \\
x_{2}(0)=0, & y_{2}(0)=0 .
\end{array}
$$

Das condições iniciais acima obtemos que $\Pi_{x}=\Gamma_{1} y_{1}+\Gamma_{2} y_{2}=0, \Pi_{y}=-\left(\Gamma_{1} x_{1}+\right.$ $\left.\Gamma_{2} x_{2}\right)=-\Gamma_{1}$ e que as equações (3.40) se escrevem como:

$$
\begin{array}{r}
x_{1}(t)-x_{2}(t)=\cos \Omega t \\
y_{1}(t)-y_{2}(t)=-\sin \Omega t
\end{array}
$$

donde obtemos que a solução do sistema (3.37):

$$
\begin{aligned}
& x_{1}(t)=\frac{\Gamma_{1}}{\Gamma_{1}+\Gamma_{2}}+\frac{\Gamma_{2}}{\Gamma_{1}+\Gamma_{2}} \cos \left(\frac{\Gamma_{1}+\Gamma_{2}}{2 \pi r_{12}^{2}} t\right), \\
& x_{2}(t)=\frac{\Gamma_{1}}{\Gamma_{1}+\Gamma_{2}}-\frac{\Gamma_{1}}{\Gamma_{1}+\Gamma_{2}} \cos \left(\frac{\Gamma_{1}+\Gamma_{2}}{2 \pi r_{12}^{2}} t\right), \\
& y_{1}(t)=-\frac{\Gamma_{2}}{\Gamma_{1}+\Gamma_{2}} \sin \left(\frac{\Gamma_{1}+\Gamma_{2}}{2 \pi r_{12}^{2}} t\right), \\
& y_{2}(t)=\frac{\Gamma_{1}}{\Gamma_{1}+\Gamma_{2}} \sin \left(\frac{\Gamma_{1}+\Gamma_{2}}{2 \pi r_{12}^{2}} t\right) .
\end{aligned}
$$

Segundo caso: $\Gamma_{1}+\Gamma_{2}=0$

Consideremos o caso que os valores das intensidade dos vórtices são $\Gamma_{1}=-\Gamma_{2}=\Gamma$. O fato de $\Pi_{x}$ e $\Pi_{y}$ serem integrais primeiras implicam neste caso que $x_{1}-x_{2}$ e $y_{1}-y_{2}$ 
são constantes do movimento. Levando em conta as equações (3.37), concluímos que os vórtices têm velocidade constante.

Em termos das condições iniciais, as trajetórias dos vórtices serão dadas por:

$$
\begin{aligned}
& x_{1}(t)=x_{1}(0)+\frac{\Gamma}{2 \pi} \frac{\left(y_{1}(0)-y_{2}(0)\right)}{r_{12}^{2}(0)} t, \\
& x_{2}(t)=x_{2}(0)+\frac{\Gamma}{2 \pi} \frac{\left(y_{1}(0)-y_{2}(0)\right)}{r_{12}^{2}(0)} t, \\
& y_{1}(t)=y_{1}(0)-\frac{\Gamma}{2 \pi} \frac{\left(x_{1}(0)-x_{2}(0)\right)}{r_{12}^{2}(0)} t, \\
& y_{1}(t)=y_{2}(0)-\frac{\Gamma}{2 \pi} \frac{\left(x_{1}(0)-x_{2}(0)\right)}{r_{12}^{2}(0)} t .
\end{aligned}
$$

Assim, vemos claramente que as trajetórias dos dois vórtices com intensidade $\Gamma_{1}=$ $-\Gamma_{2}=\Gamma$ são retas paralelas. Estas trajetórias, portanto, não são limitadas, ao contrário do que acontece no caso dos dois vórtices com intensidade total não nula. Naquele caso as trajetórias, de acordo (3.43), são circunferência de centro $\left(\frac{\Gamma_{1}}{\Gamma_{1}+\Gamma_{2}}, 0\right)$ e raio $\frac{\Gamma_{2}}{\Gamma_{1}+\Gamma_{2}}$ para o primeiro vórtice, e raio $\frac{\Gamma_{1}}{\Gamma_{1}+\Gamma_{2}}$ para o segundo vórtice. Estamos, portanto em presença de uma significativa bifurcação nas órbitas do sistema de dois vórtices sem massa.

Se tomarmos as condições iniciais (3.42), tem-se a solução (3.44) na forma:

$$
\begin{aligned}
& x_{1}(t)=1, \\
& x_{2}(t)=0, \\
& y_{1}(t)=-\frac{\Gamma^{2}}{2 \pi} t, \\
& y_{2}(t)=-\frac{\Gamma^{2}}{2 \pi} t
\end{aligned}
$$

que é o limite, quando $\Gamma_{1}+\Gamma_{2} \rightarrow 0$, da expressão (3.43) desenvolvida em série de Taylor:

$$
\begin{aligned}
& x_{1}(t)=1-\Gamma_{2} \frac{\Gamma_{1}+\Gamma_{2}}{8 \pi^{2}} t^{2}+O\left(\left(\Gamma_{1}+\Gamma_{2}\right)^{3} t^{4}\right), \\
& x_{2}(t)=\Gamma_{1} \frac{\Gamma_{1}+\Gamma_{2}}{8 \pi^{2}} t^{2}+O\left(\left(\Gamma_{1}+\Gamma_{2}\right)^{3} t^{4}\right), \\
& y_{1}(t)=\frac{\Gamma_{2}}{2 \pi} t-\Gamma_{2} \frac{\left(\Gamma_{1}+\Gamma_{2}\right)^{2}}{48 \pi^{3}} t^{3}+O\left(\left(\Gamma_{1}+\Gamma_{2}\right)^{4} t^{5}\right), \\
& y_{2}(t)=-\frac{\Gamma_{1}}{2 \pi} t-\Gamma_{1} \frac{\left(\Gamma_{1}+\Gamma_{2}\right)^{2}}{48 \pi^{3}} t^{3}+O\left(\left(\Gamma_{1}+\Gamma_{2}\right)^{4} t^{5}\right) .
\end{aligned}
$$


Notemos que este limite não é uniforme no tempo, e que a convergência é de ordem dois no tempo e ordem um em $\Gamma_{1}+\Gamma_{2}$. Assim, conclui-se que a trajetória do sistema com a soma das intensidade não nula, aproximará bem a trajetória do sistema com soma das intensidade nula, quando $\Gamma_{1}+\Gamma_{2} \rightarrow 0$, para intervalos de tempo ] $-T, T$, somente para $T$ suficientemente pequeno.

\subsubsection{Exemplo 2 : três vórtices sem massa}

Estudaremos o caso de três vórtices sem massa, com intensidades $\Gamma_{1}=\Gamma_{2}=\Gamma_{3}=1$. A função Hamiltoniana deste sistema é, de acordo com (3.14),

$$
H=-\frac{1}{4 \pi}\left(\ln \left\|\vec{x}_{2}-\vec{x}_{1}\right\|+\ln \left\|\vec{x}_{3}-\vec{x}_{1}\right\|+\ln \left\|\vec{x}_{3}-\vec{x}_{2}\right\|\right)
$$

onde $\vec{x}_{i}=\left(x_{i}, y_{i}\right), i=1,2,3$, e a forma simplética é:

$$
w=d y_{1} \wedge x_{1}+d y_{2} \wedge x_{2}+d y_{3} \wedge x_{3}
$$

De acordo com (3.20), o sistema tem as sequintes integrais primeiras:

$$
\begin{aligned}
\Pi_{x} & =y_{1}+y_{2}+y_{3}, \\
\Pi_{y} & =-\left(x_{1}+x_{2}+x_{3}\right), \\
J & =-\frac{1}{2}\left(\left\|\vec{x}_{1}\right\|^{2}+\left\|\vec{x}_{2}\right\|^{2}+\left\|\vec{x}_{3}\right\|^{2}\right) .
\end{aligned}
$$

\section{REDUÇÃO:}

Definindo o centro de vorticidade por:

$$
\vec{M}_{1}=\frac{\vec{x}_{1}+\vec{x}_{2}+\vec{x}_{3}}{3}
$$

e notando que

$$
\begin{aligned}
\vec{x}_{1}-\vec{M}_{1} & =\frac{1}{2}\left[\left(\vec{x}_{1}-\vec{x}_{2}\right)+\left(\vec{M}_{1}-\vec{x}_{3}\right)\right], \\
\vec{x}_{2}-\vec{M}_{1} & =\frac{1}{2}\left[-\left(\vec{x}_{1}-\vec{x}_{2}\right)+\left(\vec{M}_{1}-\vec{x}_{3}\right)\right]
\end{aligned}
$$


definamos novas variáveis para o problema:

$$
\begin{aligned}
\vec{M}_{1} & =\left(\eta x_{0}, \eta y_{0}\right), \\
\vec{x}_{1}-\vec{x}_{2} & =\alpha \sqrt{\tilde{x}_{1}}\left(\cos \theta_{1}, \sin \theta_{1}\right), \\
\vec{M}_{1}-\vec{x}_{3} & =\beta \sqrt{\tilde{x}_{2}}\left(\cos \theta_{2}, \sin \theta_{2}\right) .
\end{aligned}
$$

Para determinar $\eta, \alpha$ e $\beta$, impomos que a transformação que leva as coordenadas $\left(\left(x_{1}, y_{1}\right),\left(x_{2}, y_{2}\right),\left(x_{3}, y_{3}\right)\right)$ a $\left.\left(x_{0}, y_{0}, \tilde{x}_{1}, \theta_{1}, \tilde{x}_{2}, \theta_{2}\right)\right)$ seja canônica. Obtemos, desta forma, que:

$$
\eta=\frac{\sqrt{3}}{3}, \quad \alpha=2, \quad \beta=\frac{2}{3} \sqrt{3} .
$$

Assim, a partir de (3.50) á (3.52), temos que a transformação canônica é:

$$
\begin{aligned}
& x_{1}=\frac{\sqrt{3}}{3} x_{0}+\sqrt{\tilde{x}_{1}} \cos \theta_{1}+\frac{\sqrt{3}}{3} \sqrt{\tilde{x}_{2}} \cos \theta_{2}, \\
& y_{1}=\frac{\sqrt{3}}{3} y_{0}+\sqrt{\tilde{x}_{1}} \sin \theta_{1}+\frac{\sqrt{3}}{3} \sqrt{\tilde{x}_{2}} \sin \theta_{2} \text {, } \\
& x_{2}=\frac{\sqrt{3}}{3} x_{0}-\sqrt{\tilde{x}_{1}} \cos \theta_{1}+\frac{\sqrt{3}}{3} \sqrt{\tilde{x}_{2}} \cos \theta_{2}, \\
& y_{2}=\frac{\sqrt{3}}{3} y_{0}-\sqrt{\tilde{x}_{1}} \sin \theta_{1}+\frac{\sqrt{3}}{3} \sqrt{\tilde{x}_{2}} \sin \theta_{2} \text {, } \\
& x_{3}=\frac{\sqrt{3}}{3} x_{0} \quad-\frac{2 \sqrt{3}}{3} \sqrt{\tilde{x}_{2}} \cos \theta_{2}, \\
& y_{3}=\frac{\sqrt{3}}{3} x_{0} \quad-\frac{2 \sqrt{3}}{3} \sqrt{\tilde{x}_{2}} \sin \theta_{2} \text {. }
\end{aligned}
$$

A hamiltoniana (3.47), nas novas coordenadas é:

$$
H=-\frac{1}{4 \pi} \ln \left[\tilde{x}_{1}\left(\tilde{x}_{1}+3 \tilde{x}_{2}\right)^{2}-12 \tilde{x}_{1}^{2} \tilde{x}_{2} \cos ^{2}\left(\theta_{1}-\theta_{2}\right)\right]
$$

e a forma simplética (3.48) é:

$$
w=d y_{0} \wedge d x_{0}+d \theta_{1} \wedge d \tilde{x}_{1}+d \theta_{2} \wedge d \tilde{x}_{2}
$$

Notemos que a Hamiltoniana $H$, definida em (3.55), não depende de $x_{0}$ e $y_{0}$, assim $x_{0}$ e $y_{0}$ são integrais primeiras do sistema (correspondentes à conservação de $\Pi_{x}$ e $\Pi_{y}$ ). 
Além disso, $H$ depende de $\theta_{1}$ e $\theta_{2}$ apenas por sua diferença, o que implica que $\tilde{x}_{1}+\tilde{x}_{2}$ também é uma integral primeira do sistema dos três vórtices.

Se fizermos uma nova transformação canônica definida por:

$$
\begin{aligned}
& p_{1}=\tilde{x}_{1}, \\
& p_{2}=\tilde{x}_{1}+\tilde{x}_{2}, \\
& q_{1}=\theta_{1}-\theta_{2}, \\
& q_{2}=\theta_{2},
\end{aligned}
$$

a Hamiltoniana (3.55), nas novas variáveis $\left(p_{1}, q_{1}, p_{2}, q_{2}\right)$ será expressa como:

$$
H=-\frac{1}{4 \pi} \ln \left[p_{1}\left(p_{1}+3\left(p_{2}-p_{1}\right)\right)^{2}-12 p_{1}^{2}\left(p_{2}-p_{1}\right) \cos ^{2} q_{1}\right],
$$

e, usando que $x_{0}$ e $y_{0}$ são integrais primeiras, a forma simplética do sistema reduzido, com 4 variáveis $p_{1}, p_{2}, q_{1}$ e $q_{2}$, é:

$$
w=d q_{1} \wedge d p_{1}+d q_{2} \wedge d p_{2}
$$

As equações do movimento para este sistema são:

$$
\begin{aligned}
& \dot{p}_{1}=\frac{\partial H}{\partial q_{1}} \\
& \dot{p}_{2}=0 \\
& \dot{q}_{1}=-\frac{\partial H}{\partial p_{1}} \\
& \dot{q}_{2}=-\frac{\partial H}{\partial p_{2}} .
\end{aligned}
$$

Se definimos

$$
V=-\left[p_{1}\left(p_{1}+3\left(p_{2}-p_{1}\right)\right)^{2}-12 p_{1}^{2}\left(p_{2}-p_{1}\right) \cos ^{2} q_{1}\right]
$$

e, dado que $H$ é conservado ao longo das soluções (3.60), introduzimos o novo tempo:

$$
\tau=\frac{1}{4 \pi} \exp (4 \pi H) t
$$


o sistema (3.60) pode ser escrito como:

$$
\begin{array}{lll}
\frac{\partial p_{1}}{\partial \tau}=\frac{\partial V}{\partial q_{1}}, & \frac{\partial q_{1}}{\partial \tau}=-\frac{\partial V}{\partial p_{1}} \\
\frac{\partial p_{2}}{\partial \tau}=0, & \frac{\partial q_{2}}{\partial \tau}=-\frac{\partial V}{\partial p_{2}} .
\end{array}
$$

Além disso, devido à (3.52), $\tilde{x_{1}}, \tilde{x_{2}}$ são positivas, donde, de (3.57), consideramos a função $V$ restrita ao conjunto:

$$
\left\{\left(p_{1}, p_{2}, q_{1}, q_{2}\right) \in \mathbb{R}^{4}: 0<\mathrm{p}_{1}<\mathrm{p}_{2}\right\}
$$

Agora, reduziremos o problema dos três vórtices, com intensidade unitária, a um sistema Hamiltoniano plano que admite conexões selas.

Como $p_{2}$ é constante através das soluções de (3.63), a integração deste sistema é equivalente á integração do sistema:

$$
\begin{aligned}
& \frac{\partial p_{1}}{\partial \tau}=-24 p_{1}^{2}\left(\mu-p_{1}\right) \cos q_{1} \sin q_{1} \\
& \frac{\partial q_{1}}{\partial \tau}=\cos ^{2} q_{1}\left(36 p_{1}^{2}-24 \mu p_{1}\right)+\left(3 \mu-2 p_{1}\right)\left(3 \mu-6 p_{1}\right)
\end{aligned}
$$

com $p_{2}=\mu$ sendo um parâmetro positivo. Os pontos de equilíbrio do sistema (3.65) que satisfazem (3.64) são:

$$
\begin{aligned}
z_{1} & =\left(p_{1}=\frac{1}{2} \mu, \cos q_{1}=0\right), \\
z_{2} & =\left(p_{1}=\frac{3}{4} \mu, \sin q_{1}=0\right), \\
z_{3} & =\left(p_{1}=\frac{1}{4} \mu, \sin q_{1}=0\right) .
\end{aligned}
$$

As matrizes do sistema (3.65) linearizado em torno dos pontos de equilíbrio $z_{1}, z_{2}$ e $z_{3}$ são, respectivamente:

$$
\left.\mathbf{M}\right|_{z_{1}}=\left(\begin{array}{cc}
0 & 3 \mu^{3} \\
-12 \mu & 0
\end{array}\right) \text {, }
$$




$$
\begin{aligned}
\left.\mathbf{M}\right|_{z_{2}} & =\left(\begin{array}{cc}
0 & -\frac{27}{8} \mu^{3} \\
9 \mu & 0
\end{array}\right), \\
\left.\mathbf{M}\right|_{z_{3}} & =\left(\begin{array}{cc}
0 & -\frac{9}{8} \mu^{3} \\
-29 \mu & 0
\end{array}\right) .
\end{aligned}
$$

Vemos claramente que os determinantes satisfazem $\left.\operatorname{Det} \mathbf{M}\right|_{z_{1}}>0,\left.\operatorname{Det} \mathbf{M}\right|_{z_{2}}>0,\left.\operatorname{Det} \mathbf{M}\right|_{z_{1}}<$ 0 , e, como o traço destas matrizes é nulo, isto implica que os valores próprios são imaginários puros no caso de $z_{1}$ e $z_{2}$, enquanto que são reais e de sinais opostos no caso de $z_{3}$. Resumindo os resultados anteriores temos a seguinte proposição:

Proposição 3.4.1 O sistema de equações diferenciais (3.65), tem os pontos de equilíbrio, nas coordenadas $p_{1}, q_{1}$,

$$
\begin{aligned}
z_{1} & =\left(p_{1}=\frac{1}{2} \mu, \cos q_{1}=0\right), \\
z_{2} & =\left(p_{1}=\frac{3}{4} \mu, \sin q_{1}=0\right), \\
z_{3} & =\left(p_{1}=\frac{1}{4} \mu, \sin q_{1}=0\right) .
\end{aligned}
$$

A familia de pontos $z_{1}, z_{2}$ são centros e a familia de pontos $z_{3}$ são pontos de sela. Além disso, existem conexões selas, as quais estão nas curvas de nível da energia $V\left(p_{1}, p_{2} ; \mu\right)=$ $-\mu^{3}$.

Dado que $V$ assume o valor $-\mu^{3}$ na familia de pontos $z_{3}$, as conexões, caso existam, devem estar nas curvas de nível da energia $V\left(p_{1}, p_{2} ; \mu\right)=-\mu^{3}$, ou seja, devem satisfazer:

$$
V\left(p_{1}, q_{1}, \mu\right)+\mu^{3}=\left(p_{1}-\mu\right)\left(p_{1}-\frac{\mu}{2\left(2+\sqrt{3} \sin q_{1}\right)}\right)\left(p_{1}-\frac{\mu}{2\left(2-\sqrt{3} \sin q_{1}\right)}\right)=0 .
$$

Portanto, a curva

$$
p_{1}=\frac{\mu}{2\left(2+\sqrt{3} \sin q_{1}\right)}, \quad 0<q_{1}<\pi, \quad p_{2}=\mu,
$$

é uma conexão de sela de (3.65) contida no conjunto (3.64), já que tende para os pontos $\left(p_{1}=\frac{\mu}{4}, q_{1}=0\right)$ e $\left(p_{1}=\frac{\mu}{4}, q_{1}=\pi\right)$ quando $q_{1}$ tende para 0 e $\pi$, respectivamente. 
É de interesse considerar a solução de (3.63) que está contida no nível de energia

$$
V\left(p_{1}, q_{1}, p_{2}\right)=-\mu^{3}<0 .
$$

Esta equação pode ser explícitamente resolvida com relação a $p_{2}$ :

$$
p_{2}=\frac{2}{3} p_{1}\left(1+\cos ^{2} q_{1}\right) \pm \frac{1}{3} \sqrt{\frac{\mu^{3}-p_{1}^{3} \sin ^{2}\left(2 q_{1}\right)}{p_{1}}},
$$

sendo que esta expressão está definida para $0<p_{1} \leq \mu$. O ramo de (3.71) que contém a curva (3.69) é:

$$
p_{2}=\frac{2}{3} p_{1}\left(1+\cos ^{2} q_{1}\right)+\frac{1}{3} \sqrt{\frac{\mu^{3}-p_{1}^{3} \sin ^{2}\left(2 q_{1}\right)}{p_{1}}}=: h_{0}\left(p_{1}, q_{1}, \mu\right) .
$$

Como numa vizinhança da separatriz (3.69) tem-se $\frac{\partial V}{\partial p_{2}} \neq 0$, a solução do sistema (3.63) satisfazendo (3.70), (3.72) e cujas órbitas estão perto de (3.69), podem ser parametrizadas por $q_{2}$, e satisfazem o sistema:

$$
\begin{aligned}
& \frac{d p_{1}}{d q_{2}}=\frac{\partial h_{0}}{\partial q_{1}}\left(p_{1}, q_{1}, \mu\right), \\
& \frac{d q_{1}}{d q_{2}}=-\frac{\partial h_{0}}{\partial p_{1}}\left(p_{1}, q_{1}, \mu\right) .
\end{aligned}
$$

A solução do sistema (3.73), tendo como órbita a curva (3.69), é obtida por integração da equação:

$$
\begin{aligned}
\frac{d q_{1}}{d q_{2}} & =-\frac{\partial h_{0}}{\partial p_{1}}\left(p_{1}=\frac{\mu}{2\left(2+\sqrt{3} \sin q_{1}\right)}, q_{1}, \mu\right) \\
& =\frac{\left(\partial V / \partial p_{1}\right)\left(p_{1}=\mu /\left(2\left(2+\sqrt{3} \sin q_{1}\right)\right), q_{1}, \mu\right)}{\left(\partial V / \partial p_{2}\right)\left(p_{1}=\mu /\left(2\left(2+\sqrt{3} \sin q_{1}\right)\right), q_{1}, \mu\right)} \\
& =\frac{2 \sin q_{1}\left(\sqrt{3}+\sin q_{1}\right)\left(2+\sqrt{3} \sin q_{1}\right)}{4+\sin ^{2} q_{1}+3 \sqrt{3} \sin q_{1}} .
\end{aligned}
$$

Portanto, temos:

$$
\begin{aligned}
q_{2}\left(q_{1}\right)-q_{2}^{0}= & \int_{q_{1}^{0}}^{q_{1}} \frac{4+\sin ^{2} t+3 \sqrt{3} \sin t}{2 \sin t(\sqrt{3}+2 \sin t)+(2+\sqrt{3} \sin t)} d t \\
= & \frac{1}{2 \sqrt{3}} \log \left\{\frac{\tan ^{2}\left(q_{1} / 2\right) \sqrt{3}+\tan \left(q_{1} / 2\right)(1 / \sqrt{3})+\tan \left(q_{1} / 2\right)}{\tan ^{2}\left(q_{1}^{0} / 2\right) \sqrt{3}+\tan \left(q_{1}^{0} / 2\right)(1 / \sqrt{3})+\tan \left(q_{1}^{0} / 2\right)}\right\} \\
& -\arctan \left(\sqrt{3}+2 \tan \left(q_{1} / 2\right)\right)+\arctan \left(\sqrt{3}+2 \tan \left(q_{1}^{0} / 2\right)\right) .
\end{aligned}
$$


Se denotarmos:

$$
\begin{array}{llrl}
q_{1} & =x+\frac{\pi}{2}, & -\frac{\pi}{2}<x<\frac{\pi}{2}, \\
q_{0}=x_{0}+\frac{\pi}{2}, & -\frac{\pi}{2}<x_{0}<\frac{\pi}{2},
\end{array}
$$

temos

$$
\begin{aligned}
q_{2}\left(x, x_{0} ; q_{2}^{0}\right)= & q_{2}\left(x+\frac{\pi}{2}\right)=q_{2}^{0}+\frac{1}{2 \sqrt{3}} \log \frac{F(x)}{F\left(x_{0}\right)} \\
& -\arctan \left(\frac{(2+\sqrt{3})+(2-\sqrt{3}) \tan (x / 2)}{1-\tan (x / 2)}\right) \\
& +\arctan \left(\frac{(2+\sqrt{3})+(2-\sqrt{3}) \tan \left(x_{0} / 2\right)}{1-\tan \left(x_{0} / 2\right)}\right) .
\end{aligned}
$$

Lembrando a identidade trigonométrica:

$$
\arctan \left(\frac{u+v}{1-u v}\right)=\arctan u+\arctan v
$$

obtemos então

$$
\begin{aligned}
q_{2}\left(x, x_{0} ; q_{2}^{0}\right)= & \left.q_{2}^{0}+\frac{1}{2 \sqrt{3}} \log \frac{F(x)}{F\left(x_{0}\right)}-\arctan (2-\sqrt{3}) \tan (x / 2)\right) \\
& \left.+\arctan (2-\sqrt{3}) \tan \left(x_{0} / 2\right)\right)
\end{aligned}
$$

com

$$
F(x)=\frac{(1+\tan (x / 2))^{2}}{(1-\tan (x / 2))^{2}} \frac{1+\sqrt{3}-(\sqrt{3}-1) \tan (x / 2)}{1+\sqrt{3}+(\sqrt{3}-1) \tan (x / 2)}
$$

Se definirmos

$$
s(x)=\frac{1}{2 \sqrt{3}} \log F(x)-\arctan [(2-\sqrt{3}) \tan (x / 2)],
$$

temos

$$
q_{2}\left(x, x_{0} ; q_{2}^{0}\right)=q_{2}^{0}+s(x)-s\left(x_{0}\right)
$$

Note que $F(-x)=(F(x))^{-1}$, donde $s(-x)=-s(x)$. 


\subsubsection{Exemplo 3 : quatro vórtices sem massa}

Nesta subseção, seguiremos de perto o artigo [5]: "The four positive vortices problems; regions of caotics behavior and non-integrability", de Castlla, M., Moauro, V., Negrini, P. e Oliva, W..

A função Hamiltoniana associada ao problema dos quatro vórtices $\vec{x}_{j}=\left(x_{j}, x_{j}\right), j=$ $1,2,3,4$. com intensidade $(1,1,1, \varepsilon), \varepsilon>0$, é dada por

$$
\begin{aligned}
H=-\frac{1}{4 \pi}\left[\ln \left\|\vec{x}_{2}-\vec{x}_{1}\right\|+\ln \left\|\vec{x}_{3}-\vec{x}_{1}\right\|+\ln \left\|\vec{x}_{3}-\vec{x}_{2}\right\|+\right. \\
\left.\varepsilon\left(\ln \left\|\vec{x}_{2}-\vec{x}_{1}\right\|+\ln \left\|\vec{x}_{3}-\vec{x}_{1}\right\|+\ln \left\|\vec{x}_{3}-\vec{x}_{2}\right\|\right)\right] \\
=H_{0}+\varepsilon H_{1}
\end{aligned}
$$

onde $H_{0}$ é a função Hamiltoniana do problema de três vórtices com intensidade unitária, definido em (3.47). E a forma simplética é dada por:

$$
w=d y_{1} \wedge d x_{1}+d y_{2} \wedge d x_{2}+d y_{3} \wedge d x_{3}+\varepsilon d y_{4} \wedge d x_{4} .
$$

De acordo com (3.20), o sistema tem as integrais primeiras

$$
\begin{aligned}
\Pi_{x} & =y_{1}+y_{2}+y_{3}+\varepsilon y_{4}, \\
\Pi_{y} & =-\left(x_{1}+x_{2}+x_{3}+\varepsilon x_{4}\right), \\
J & =-\frac{1}{2}\left(\left\|\vec{x}_{1}\right\|^{2}+\left\|\vec{x}_{2}\right\|^{2}+\left\|\vec{x}_{3}\right\|^{2}+\varepsilon\left\|\vec{x}_{4}\right\|^{2}\right) .
\end{aligned}
$$

\section{REDUÇÃO:}

Sejam:

$$
\begin{aligned}
& \mathbf{M}_{0}=\frac{\vec{x}_{1}+\vec{x}_{2}+\vec{x}_{3}+\varepsilon \vec{x}_{4}}{3+\varepsilon}, \\
& \mathbf{M}_{1}=\frac{\vec{x}_{1}+\vec{x}_{2}+\vec{x}_{3}}{3} .
\end{aligned}
$$

Vamos definir novas variáveis por:

$$
\mathbf{M}_{0}=\left(\eta_{0} x_{0}, \eta_{0} y_{0}\right)
$$




$$
\begin{aligned}
\vec{x}_{1}-\vec{x}_{2} & =\alpha \sqrt{\tilde{x}_{1}}\left(\cos \theta_{1}, \sin \theta_{1}\right), \\
\mathbf{M}_{1}-\vec{x}_{3} & =\beta \sqrt{\tilde{x}_{2}}\left(\cos \theta_{2}, \sin \theta_{2}\right), \\
\mathbf{M}_{1}-\vec{x}_{4} & =\gamma \sqrt{\tilde{x}_{3}}\left(\cos \theta_{3}, \sin \theta_{3}\right),
\end{aligned}
$$

onde $\eta_{0}, \alpha, \beta$ e $\gamma$ tomam os valores

$$
\eta_{0}=\sqrt{\frac{1}{3+\varepsilon}}, \quad \alpha=2, \quad \beta=\frac{2}{3} \sqrt{3}, \quad \gamma=\sqrt{\frac{2(3+\varepsilon)}{3}} .
$$

Desta forma, a transformação que leva as coordenadas $\left(\left(x_{1}, y_{1}\right),\left(x_{2}, y_{2}\right),\left(x_{3}, y_{3}\right)\right.$, $\left.\left(\sqrt{\varepsilon} x_{4}, \sqrt{\varepsilon} y_{4}\right)\right)$ em $\left(x_{0}, y_{0}, \tilde{x}_{1}, \theta_{1}, \tilde{x}_{2}, \theta_{2}, \tilde{x}_{3}, \theta_{3}\right)$ é canônica. Obtemos que a função Hamiltoniana (3.80) nas novas variáveis é:

$$
H=H\left(\tilde{x}_{1}, \theta_{1}, \tilde{x}_{2}, \theta_{2}, \tilde{x}_{3}, \theta_{3}\right)=H_{0}\left(\tilde{x}_{1}, \theta_{1}, \tilde{x}_{2}, \theta_{2}\right)+\varepsilon H_{1}\left(\tilde{x}_{1}, \theta_{1}, \tilde{x}_{2}, \theta_{2}, \tilde{x}_{3}, \theta_{3}\right)
$$

com $H_{0}$, definida em (3.55) e

$$
H_{1}=-\frac{1}{4 \pi} \ln \left[\frac{8 \tilde{x}_{3}+\varepsilon \phi_{1}+\varepsilon^{3 / 2} \phi_{2}}{\varepsilon^{3}}\right],
$$

onde $\phi_{1}=\phi_{1}\left(\tilde{x}_{1}, \theta_{1}, \tilde{x}_{2}, \theta_{2}, \tilde{x}_{3}, \theta_{3}\right)$ e $\phi_{2}=\phi_{2}\left(\tilde{x}_{1}, \theta_{1}, \tilde{x}_{2}, \theta_{2}, \tilde{x}_{3}, \theta_{3}\right)$. Em particular,

$$
\phi_{1}=-16 \tilde{x}_{2} \tilde{x}_{3}^{2} \cos \left(\theta_{3}-\theta_{2}\right)+8 \tilde{x}_{3}^{2}+8 \tilde{x}_{2} \tilde{x}_{3}^{2}+8 \tilde{x}_{1} \tilde{x}_{3}^{2}-16 \tilde{x}_{1} \tilde{x}_{3}^{2} \cos \left(\theta_{1}-\theta_{3}\right) .
$$

Nas novas variáveis, a forma simplética é

$$
w=d y_{0} \wedge d x_{0}+d \theta_{1} \wedge \tilde{x}_{1}+d \theta_{2} \wedge \tilde{x}_{2}+d \theta_{3} \wedge \tilde{x}_{3} .
$$

Denotando $\sigma(\varepsilon)=\varepsilon^{3 / 2}$ e fazendo a transformação canônica

$$
\begin{array}{ll}
p_{1}=\tilde{x}_{1}, & q_{1}=\theta_{1}-\theta_{2}, \\
p_{2}=\tilde{x}_{1}+\tilde{x}_{2}, & q_{2}=\theta_{2}-\theta_{3}, \\
p_{3}=\tilde{x}_{1}+\tilde{x}_{2}+\tilde{x}_{3}, & q_{3}=\theta_{3},
\end{array}
$$

temos que a função Hamiltoniana (3.85) nestas novas variáveis é:

$$
\begin{aligned}
H & =H_{0}+\varepsilon H_{1} \\
& =-\frac{1}{4 \pi}\left[\ln (-V)+\varepsilon \ln \left(\frac{8\left(p_{3}-p_{2}\right)^{3}+\varepsilon \phi_{1}+\sigma(\varepsilon)}{\varepsilon^{3}}\right)\right],
\end{aligned}
$$


onde $V=V\left(p_{1}, q_{1}, p_{2}\right)$ é definido em (3.61) e $\phi_{1}, \sigma$ são expressas nas novas coordenadas. Em particular, $\phi_{1}$, definida em (3.87), nas novas coordenadas é dada por:

$$
\begin{aligned}
\phi_{1}= & -16\left(p_{3}-p_{2}\right)^{2}\left(p_{2}-p_{1}\right) \cos ^{2} q_{2}-8\left(p_{3}-p_{2}\right)^{3}+8\left(p_{3}-p_{2}\right)^{2}\left(p_{2}-p_{1}\right) \\
& +8 p_{1}\left(p_{3}-p_{2}\right)^{2}-16 p_{1}\left(p_{3}-p_{2}\right)^{2} \cos ^{2}\left(q_{1}+q_{2}\right) .
\end{aligned}
$$

Utilizando as propriedades logarítmicas, a Hamiltoniana (3.90) fica:

$$
H=-\frac{1}{4 \pi}\left[\ln \left(-V\left[8\left(p_{3}-p_{2}\right)^{3}+\varepsilon \phi_{1}+\sigma(\varepsilon)\right]^{\varepsilon}\right)+\varepsilon \ln \frac{1}{\varepsilon^{3}}\right] .
$$

Definindo

$$
W\left(p_{1}, q_{1}, p_{2}, q_{2}, p_{3}\right)=\left[V\left(p_{1}, q_{1}, p_{2}\right)\right]\left[8\left(p_{3}-p_{2}\right)^{3}+\varepsilon \phi_{1}+\sigma(\varepsilon)\right]^{\varepsilon},
$$

onde a função $W$, de acordo com (3.84) e (3.89), é definida para $p_{3}>p_{2}$, é $2 \pi$-periódica na variável $q_{2}$ e é independente de $q_{3}$.

Se fizermos o desenvolvimento em polinômio de Taylor da expressão $\left[8\left(p_{3}-p_{2}\right)^{3}+\right.$ $\left.\varepsilon \phi_{1}+\sigma(\varepsilon)\right]^{\varepsilon}$, para $\varepsilon$ suficientemente pequeno, obtemos:

$$
\begin{aligned}
W=V\left(p_{1}, q_{1}, p_{2}\right)\left\{1+3 \varepsilon \ln \left[2\left(p_{3}-p_{2}\right)\right]\right. \\
+\frac{\varepsilon^{2}}{2}\left[9 \ln ^{2}\left[2\left(p_{3}-p_{2}\right]+\frac{\phi_{1}}{8\left(p_{2}-p_{3}\right)^{3}}\right]\right\}+o\left(\varepsilon^{2}\right) .
\end{aligned}
$$

Assim, podemos escrever a Hamiltoniana (3.90) na forma

$$
H=-\frac{1}{4 \pi} \ln [-W]
$$

com forma simplética

$$
w=d q_{1} \wedge d p_{1}+d q_{2} \wedge d p_{1}+d q_{3} \wedge d p_{3}
$$

As equações do movimento do sistema com Hamiltoniana definida em (3.92) e forma simplética (3.93), são :

$$
\dot{p}_{1}=\frac{\partial H}{\partial q_{1}}, \quad \dot{q}_{1}=-\frac{\partial H}{\partial p_{1}}
$$




$$
\begin{array}{ll}
\dot{p}_{2}=\frac{\partial H}{\partial q_{2},} & \dot{q}_{2}=-\frac{\partial H}{\partial p_{2}}, \\
\dot{p}_{3}=0, & \dot{q}_{3}=-\frac{\partial H}{\partial p_{3}} .
\end{array}
$$

Como $H$ é conservado sobre as soluções de (3.94), podemos introduzir o novo tempo

$$
\tau=\frac{1}{4 \pi} \exp (-4 \pi H) t
$$

de maneira que o sistema (3.94) torna-se:

$$
\begin{array}{lll}
\frac{\partial p_{1}}{\partial \tau}=\frac{\partial W}{\partial q_{1}}, & \frac{\partial q_{1}}{\partial \tau}=-\frac{\partial W}{\partial p_{1}} \\
\frac{\partial p_{2}}{\partial \tau}=\frac{\partial W}{\partial q_{2}}, & \frac{\partial q_{2}}{\partial \tau}=-\frac{\partial W}{\partial p_{2}} \\
\frac{\partial p_{3}}{\partial \tau}=0, & \frac{\partial q_{3}}{\partial \tau}=-\frac{\partial W}{\partial p_{3}} .
\end{array}
$$

Este sistema tem como integrais primeiras

$$
p_{3}=\text { constante }, \quad W\left(p_{1}, q_{1}, p_{2}, q_{2} ; p_{3}, \varepsilon\right)=\text { constante }
$$

Notemos que, para $\varepsilon=0$, tem-se que $W=V\left(p_{1}, q_{1}, p_{2}\right)$, com $V$ definido em (3.61). Como as soluções (3.79) do sistema (3.63) estão no nível de energia $V\left(p_{1}, q_{1}, p_{2}\right)=-\mu^{3}<$ 0 e suas órbitas estão perto da curva (3.69), estudaremos as soluções do sistema (3.95) no nível de energia $W=-\mu^{3}<0$, numa vizinhança da curva (3.69), para $\varepsilon>0$, suficientemente pequeno. Reduziremos o sistema (3.95), a um sistema de equações diferenciais que não é mais autônomo.

Como para $\mu>0$ fixo, tem-se $\left(\partial W / \partial p_{2}\right)_{\varepsilon=0}=\partial V / \partial p_{2} \neq 0$, sobre a curva (3.69), então da equação

$$
W=W\left(p_{1}, q_{1}, p_{2}, q_{2} ; p_{3}=\mu+\alpha, \varepsilon\right)=-\mu^{3}<0,
$$

podemos obter $p_{2}$ explícitamente, para valores de $\alpha$ e $\varepsilon$ não negativos e suficientemente pequenos. Se supusermos além disso que a solução de (3.96) toma seus valores em

$$
\left\{p_{2}:\left|p_{2}-\mu\right|<\frac{\alpha}{2}\right\}
$$


podemos escrever

$$
p_{2}\left(p_{1}, q_{1}, q_{2} ; \alpha, \varepsilon, \mu\right)=h_{0}\left(p_{1}, q_{1}, \mu\right)+\varepsilon \chi_{0}+\varepsilon^{2} \chi_{0}^{\prime}+o\left(\varepsilon^{2}\right)
$$

onde $\chi_{0}^{\prime}$ é $2 \pi$-periódico em $q_{2}$ e

$$
\begin{aligned}
\chi_{0}=\left(\frac{\partial p_{2}}{\partial \varepsilon}\right)_{\varepsilon=0}= & \frac{3 \mu^{3} \ln \left[2\left(\mu+\alpha-h_{0}\left(p_{1}, q_{1} ; \mu\right)\right]\right.}{\left(\partial V / \partial p_{2}\right)_{p_{2}=h_{0}\left(p_{1}, q_{1} ; \mu\right)}} \\
\chi_{0}^{\prime}=\frac{1}{2}\left(\frac{\partial^{2} p_{2}}{\partial \varepsilon^{2}}\right)_{\varepsilon=0}= & -\frac{1}{\left(\partial V / \partial p_{2}\right)_{p_{2}=h_{0}}}\left[-\frac{9}{2} \mu^{3} \ln ^{2}\left[2\left(\mu+\alpha-h_{0}\right]\right.\right. \\
& -\frac{\mu^{3} \phi_{1}}{8\left(\mu+\alpha-h_{0}\right)^{3}}+9 \mu^{6} \frac{\ln \left[2\left(\mu+\alpha-h_{0}\right]\right.}{\left(\mu+\alpha-h_{0}\right)\left(\partial V / \partial p_{2}\right)_{p_{2}=h_{0}}} \\
& +3 \ln \left[2\left(\mu+\alpha-h_{0}\right]\left(\frac{\partial V}{\partial p_{2}}\right)_{p_{2}=h_{0}}+\frac{1}{2}\left(\frac{\partial^{2} V}{\partial p_{2}^{2}}\right)_{p_{2}=h_{0}} \chi_{0}^{2}\right] .
\end{aligned}
$$

Dos resultados acima, temos a seguinte proposição

Proposição 3.4.2 O sistema de equações diferenciais parciais (3.95), cujas soluções estão perto da curva (3.69), e no nível de energia (3.96) é reduzido ao sistema de equações diferenciais na forma

$$
\begin{aligned}
& \frac{\partial p_{1}}{\partial q_{2}}=F_{q_{1}}\left(p_{1}, q_{1}, \mu\right)+\varepsilon \chi_{q_{1}}\left(p_{1}, q_{1}, q_{2} ; \alpha, \varepsilon, \mu\right), \\
& \frac{\partial q_{1}}{\partial q_{2}}=-F_{p_{1}}\left(p_{1}, q_{1}, \mu\right)-\varepsilon \chi_{q_{1}}\left(p_{1}, q_{1}, q_{2} ; \alpha, \varepsilon, \mu\right)
\end{aligned}
$$

para $\varepsilon$ suficientemente pequeno, onde $\left(F_{q_{1}}, F_{p_{1}}\right)=\left(\partial h_{0} / \partial q_{1}, \partial h_{0} / \partial p_{1}\right)$ e $\chi=\chi_{0}+\varepsilon \chi_{0}^{\prime}+$ $o(\varepsilon)$ é $2 \pi$ periódica em $q_{2}$.

Assim, o problema em estudo foi reduzido ao problema de perturbação de um sistema de equações diferenciais ordinárias. Quando $\varepsilon=0$, o sistema (3.99) é reduzido ao sistema (3.73), o qual descreve o movimento de três vórtices sem massa, cuja solução representada por $p_{1}=p_{1}\left(q_{2}-q_{2}^{0}\right), q_{1}=q_{1}\left(q_{2}-q_{2}^{0}\right)$, definida em (3.69) e (3.79), com $x_{0}=0$, é

$$
\begin{aligned}
q_{2}-q_{2}^{0} & =s(x), \\
p_{1} & =\frac{\mu}{2(2+\sqrt{3} \cos x)}, \quad x \in\left(-\frac{\pi}{2}, \frac{\pi}{2}\right)
\end{aligned}
$$


onde $q_{1}=x+\frac{\pi}{2}$ e $s(x)$ esta definido em (3.78).

Assim, estão satisfeitas as condições para utilizar o método de Melnikov (ver [8],[15] ) para estudar o comportamento das conexões de selas ligando os pontos de coordenadas $\left(p_{1}, q_{1}\right): \bar{z}=(\mu / 4,0)$ e $z^{*}=(\mu / 4, \pi)$. Comecemos por definir a função de Melnikov relacionada ao sistema $(3.99)$

$$
\mathbf{I}\left(q_{2}^{0}\right)=\int_{-\infty}^{\infty} F\left(p_{1}\left(q_{2}-q_{2}^{0}\right), q_{1}\left(q_{2}-q_{2}^{0}\right) ; \mu\right) \times \chi\left(p_{1}\left(q_{2}-q_{2}^{0}\right), q_{1}\left(q_{2}-q_{2}^{0}\right), q_{2} ; \alpha, \varepsilon, \mu\right) d q_{2}
$$

onde utilizou-se a notação $F \times \chi=F_{q_{1}} \chi_{p_{1}}-F_{p_{1}} \chi_{q_{1}}$ e $\left(p_{1}\left(q_{2}-q_{2}^{0}\right), q_{1}\left(q_{2}-q_{2}^{0}, q_{2}\right)\right)$ é a órbita heteroclínica ligando os pontos $\bar{z}$ e $z^{*}$.

Com auxílio desta função, podemos mostrar a existência de pontos heteroclínicos transversos e consequentemente provar a existência de "horseshoes". De fato temos o seguinte resultado, devido a Melnikov.

Teorema 3.4.3 Método de Melnikov. Se a função $\mathbf{I}\left(q_{0}\right)$ calculada ao longo da órbita pertencente à interseção da variedade estável de $z^{*}$ com a variedade instável de $\bar{z}$, tem zero simples e é limitada, então para $\varepsilon$ suficientemente pequeno, as variedades $W^{s}\left(z^{*}\right)$ e instável $W^{u}(\bar{z})$, interseptam-se transversalmnte.

Se mudamos de variável na integral de Melnikov (3.101), subtituindo $q_{2}=q_{2}(x)$ e se denotamos $z=\left(p_{1}, q_{1}\right)$ a órbita parametrizada, pela expressão (3.100), obtemos (3.101) na forma:

$$
\begin{aligned}
\mathbf{I}\left(\mathbf{q}_{2}^{\mathbf{0}}\right) & =\int_{-\pi / 2}^{\pi / 2}\left[\chi_{q_{1}}\left(z(x), q_{2}(x) ; \alpha, \varepsilon, \mu\right)-\frac{F_{q_{1}}(x)}{F_{p_{1}}(x)} \chi_{p_{1}}\left(z(x), q_{2}(x) ; \alpha, \varepsilon, \mu\right)\right] d x \\
& =\int_{-\pi / 2}^{\pi / 2} \frac{d}{d x}\left[\chi\left(z(x), q_{2}(x) ; \alpha, \varepsilon, \mu\right)\right] d x-\int_{-\pi / 2}^{\pi / 2} \frac{\partial}{\partial q_{2}} \chi\left(z(x), q_{2}(x) ; \alpha, \varepsilon, \mu\right) d x \\
& =\int_{-\pi / 2}^{0} \frac{\partial}{\partial q_{2}} \chi\left(\bar{z}, q_{2}(x) ; \alpha, \varepsilon, \mu\right) \frac{\partial q_{2}}{\partial x} d x+\int_{0}^{\pi / 2} \frac{\partial}{\partial q_{2}} \chi\left(z^{*}, q_{2}(x) ; \alpha, \varepsilon, \mu\right) \frac{\partial q_{2}}{\partial x} d x \\
& -\int_{-\pi / 2}^{\pi / 2}\left\{\frac{\partial}{\partial q_{2}} \chi\left(z(x), q_{2}(x) ; \alpha, \varepsilon, \mu\right) \frac{\partial q_{2}}{\partial x}\right\}_{z=z(x)} d x
\end{aligned}
$$

$\operatorname{com} \bar{z}=\left(\frac{\mu}{4}, 0\right), z^{*}=\left(\frac{\mu}{4}, \pi\right)$. 
De (3.98) vem que:

$$
\begin{aligned}
\frac{\partial \chi}{\partial q_{2}} & =\varepsilon \frac{\partial}{\partial q_{2}} \chi_{o}^{\prime}\left(p_{1}, q_{1}, q_{2} ; \alpha, \mu\right)+o(\varepsilon) \\
& =-\frac{1}{8} \frac{1}{\left(\partial V / \partial p_{2}\right)_{p_{2}=h_{0}\left(p_{1}, q_{1} ; \mu\right)}} \mu^{3} \varepsilon \frac{1}{\left[\mu+\alpha-h_{0}\left(p_{1}, q_{1} ; \mu\right)\right]^{3}}\left(\frac{\partial \phi_{1}^{R}}{\partial q_{2}}\right)_{p_{2}=h_{0}\left(p_{1}, q_{1} ; \mu\right)},
\end{aligned}
$$

onde

$$
\phi_{1}^{R}=-16\left(\mu+\alpha-p_{2}\right)^{2}\left[\left(p_{2}-p_{1}\right) \cos ^{2} q_{2}+p_{1} \cos ^{2}\left(q_{1}+q_{2}\right)\right] .
$$

Como ao longo do movimento $(3.100), h_{0}\left(p_{1}, q_{1} ; \mu\right)=\mu$, se fixamos $\alpha=\frac{1}{2}$, tem-se

$$
\left[\frac{\partial}{\partial q_{2}} \chi\right]_{z=z(x)}=-\frac{\mu^{3} \varepsilon}{\left(\partial V / \partial p_{2}\right)_{z=z(x), p_{2}=\mu}} \frac{\partial \phi_{1}^{R}}{\partial q_{2}}\left(p_{1}(x), q_{1}(x) ; \alpha, \mu\right),
$$

com

$$
\phi_{1}^{R}=-4\left[\left(\mu-p_{1}\right) \cos ^{2} q_{2}+p_{1} \cos ^{2}\left(q_{1}+q_{2}\right)\right]
$$

e,

$$
\left(\frac{\partial V}{\partial p_{2}}\right)_{z=z(x), p_{2}=\mu}=-\frac{3 \mu^{2}\left(4+\sqrt{3} \cos x+\cos ^{2} x\right)}{(2+\sqrt{3} \cos x)^{2}} .
$$

Por outro lado, temos:

$$
\left[\frac{\partial}{\partial q_{2}} \chi\right]_{z=\bar{z}}=\left[\frac{\partial}{\partial q_{2}} \chi\right]_{z=z^{*}}=\frac{4}{3} \mu^{2} \varepsilon \sin \left(2 q_{2}(x)\right)
$$

e,

$$
\frac{\partial q_{2}}{\partial q_{1}}=\frac{4+\cos ^{2} x+3 \sqrt{3} \cos (x)}{2 \cos x(\sqrt{3}+2 \cos x)(2+\sqrt{3} \cos x)} .
$$

Com estes resultados podemos escrever (3.102), na forma

$$
\begin{aligned}
\mathbf{I}\left(\mathbf{q}_{2}^{\mathbf{0}}\right)= & -4 \mu^{3} \varepsilon \int_{-\pi / 2}^{\pi / 2}\left\{\frac{4+\cos ^{2} x+3 \sqrt{3} \cos x}{6 \mu \cos x(\sqrt{3}+2 \cos x)(2+\sqrt{3} \cos x)} \sin \left(2 q_{2}^{0}+2 s(x)\right)\right. \\
& -\frac{2+\sqrt{3}}{12 \mu \cos x(\sqrt{3}+2 \cos x)}\left[\left(2-\frac{1}{2+\sqrt{3} \cos x}\right) \sin \left(2 q_{2}^{0}+2 s(x)\right)\right. \\
& \left.\left.-\frac{1}{2+\sqrt{3} \cos x} \sin \left(2 q_{2}^{0}+2 x+2 s(x)\right)\right]\right\} d x+O(\varepsilon) .
\end{aligned}
$$


Finalmente, agrupando os fatores $\sin \left(2 q_{2}^{0}\right)$ e $\cos \left(2 q_{2}^{0}\right)$, em (3.103), obtemos

$$
\mathbf{I}\left(\mathbf{q}_{\mathbf{2}}^{0}\right)=\frac{2}{3} \mu^{2} \varepsilon\left[\mathbf{I}_{\mathbf{1}} \sin \left(2 q_{2}^{0}\right)+\mathbf{I}_{\mathbf{2}} \cos \left(2 q_{2}^{0}\right)\right]+O(\varepsilon)
$$

onde $\mathbf{I}_{1}$ e $\mathbf{I}_{2}$ não dependem de $q_{2}^{0}$.

Para garantir a existência de interseção transversal entre as variedades estável e instável é suficiente que a expressão

$$
\mathbf{I}_{1} \sin \left(2 q_{2}^{0}\right)+\mathbf{I}_{2} \cos \left(2 q_{2}^{0}\right)
$$

tenha um zero simples, e para isto, basta comprovar que $\mathbf{I}_{\mathbf{1}}$ ou $\mathbf{I}_{\mathbf{2}}$ seja não nulo, segundo o próximo lema.

Lema 3.4.1 Se $\mathbf{I}_{1}$ ou $\mathbf{I}_{2}$ for não nulo, então a função de Melnikov tem um zero simples.

Demostração: É claro que existem zeros da função acima. Suponha que exista um zero $p_{2}^{0}$, mas que este não seja simples. Nestas condições, verificam-se simultaneamente as equações:

$$
\begin{aligned}
& \mathbf{I}_{\mathbf{1}} \sin \left(2 p_{2}^{0}\right)+\mathbf{I}_{\mathbf{2}} \cos \left(2 p_{2}^{0}\right)=0 \\
& \mathbf{I}_{\mathbf{1}} \cos \left(2 p_{2}^{0}\right)-\mathbf{I}_{\mathbf{2}} \sin \left(2 p_{2}^{0}\right)=0
\end{aligned}
$$

o que implica que $\mathbf{I}_{1}=\mathbf{I}_{2}=0$, contradizendo a hipótese original.

Portanto uma condição suficiente, para mostrar a existência de pontos heteroclínicos transversais, é mostrar que $\mathbf{I}_{\mathbf{1}}$ não se anula. A integral $\mathbf{I}_{\mathbf{1}}$ é dada por

$$
\left.\mathbf{I}_{\mathbf{1}}=-2 \sqrt{3} \int_{0}^{\pi / 2}\left\{\frac{\cos ^{2} x \cos (2 s(x))}{(\sqrt{3}+2 \cos x)(2+\sqrt{3} \cos x}\right)-\frac{\sqrt{3} \sin x \sin (2 s(x))}{3(\sqrt{3}+2 \cos x)}\right\} d x .
$$

Esta integral foi calculada por métodos numéricos, mostrando não ser nula (ver [5]). O valor obtido para $\mathbf{I}_{1}$ foi 0.2621 , com um erro de $10^{-4}$. Assim, foi provada a existência de pontos heteroclínicos transversais e, consequentemente, a existência de comportamento caótico. Temos portanto o seguinte resultado. 
Teorema 3.4.4 O sistema Hamiltoniano $\left(\mathbb{R}^{8}, \mathrm{w}, \mathrm{H}\right)$ de quatro vórtices com intensidade $(1,1,1, \varepsilon)$, com função Hamiltoniana $H$ definida em (3.80) e forma simplética $w$, definida em (3.81), tem pontos heteroclínicos transversais para valores de $\varepsilon$ suficientemente pequenos.

Este resultado implica que há novas regiões de comportamento caótico para o problema dos quatro vórtices com massa, com intensidades positivas, diferentes das encontradas antes por Ziglin (ver [29]). Em particular, esta é outra demostração da não integrabilidade do problema.

Ziglin considera o problema restrito de quatro vórtices: um vórtice com intensidade tão pequena que não afeta o movimento dos outros vórtices, mas que se movimenta pela ação do campo de velocidades dos outros três vórtices, de intensidade unitária. As equações do problema restrito são:

$$
\begin{aligned}
& \dot{x}_{4}=-\frac{1}{2 \pi} \sum_{j=1}^{3} \frac{y_{4}-y_{j}(t)}{\mathrm{r}_{4 j}} \\
& \dot{y}_{4}=\frac{1}{2 \pi} \sum_{j=1}^{3} \frac{x_{4}-x_{j}(t)}{\mathrm{r}_{4 j}}
\end{aligned}
$$

onde $\mathbf{r}_{4 j}=\sqrt{\left(x_{4}-x_{j}\right)^{2}+\left(y_{4}-y_{j}\right)^{2}}, j=1,2,3,\left(x_{4}, y_{4}\right)$ corresponde às coordenadas do vórtice com intensidade 0 e $\left(x_{j}, y_{j}\right), j=1,2,3$ são as coordenadas obtidas da solução do problema do movimento dos três vórtices com intensidade $(1,1,1)$. Estas soluções serão usadas numa forma conveniente. Se denotamos por $\mathbf{a}_{\mathbf{i}}$ os lados do triângulo determinado pelos três vórtices e $A_{i}, i=1,2,3$ seus ângulos opostos, então temos as seguintes identidades:

$$
\mathrm{a}_{1}^{2}=\left|\mathrm{r}_{23}\right|^{2}, \quad \mathrm{a}_{2}^{2}=\left|\mathrm{r}_{13}\right|^{2}, \quad \mathrm{a}_{3}^{2}=\left|\mathrm{r}_{12}\right|^{2}
$$

e

$$
\sin A_{1}=\frac{\left|\mathbf{a}_{2} \times \mathbf{a}_{3}\right|}{\left|\mathbf{a}_{2}\right|\left|\mathbf{a}_{3}\right|}, \quad \sin A_{2}=\frac{\left|\mathbf{a}_{1} \times \mathbf{a}_{3}\right|}{\left|\mathbf{a}_{1}\right|\left|\mathbf{a}_{3}\right|}, \quad \sin A_{3}=\frac{\left|\mathbf{a}_{1} \times \mathbf{a}_{2}\right|}{\left|\mathbf{a}_{1}\right|\left|\mathbf{a}_{2}\right|}
$$


Derivando a expressão (3.109), com respeito ao tempo, e utilizando as equações do movimento dos vórtices com intensidade unitária $(1,1,1)$,

$$
\begin{aligned}
& \dot{x}_{j}=\frac{\partial H_{0}}{\partial y_{j}}, \\
& \dot{y}_{j}=-\frac{\partial H_{0}}{\partial x_{j}}, \quad j=1,2,3,
\end{aligned}
$$

com $H_{0}$ definida em (3.47), obtem-se as equações do problema restrito dos três vórtices, na forma:

$$
\begin{aligned}
& \dot{a}_{1}=\frac{1}{2 \pi}\left(\frac{\sin A_{3}}{a_{2}}-\frac{\sin A_{2}}{a_{3}}\right) \\
& \dot{a}_{2}=\frac{1}{2 \pi}\left(\frac{\sin A_{1}}{a_{3}}-\frac{\sin A_{3}}{a_{1}}\right) \\
& \dot{a}_{3}=\frac{1}{2 \pi}\left(\frac{\sin A_{2}}{a_{1}}-\frac{\sin A_{1}}{a_{2}}\right)
\end{aligned}
$$

O sistema (3.112) admite duas integrais primeiras, as quais são expressas em termos dos lados do triângulo determinado pelos vórtices. De fato, se subtituimos as identidades,

$$
A=\frac{1}{2} a_{2} a_{3} \sin A_{1}=\frac{1}{2} a_{1} a_{3} \sin A_{2}=\frac{1}{2} a_{1} a_{2} \sin A_{3},
$$

onde $A$ é área do triângulo determinado pelos três vórtices, nas equações (3.112), obtemos o seguinte sistema:

$$
\begin{aligned}
& a_{1} \dot{a}_{1}=\frac{A}{2 \pi}\left(a_{2}^{-2}-a_{3}^{-2}\right), \\
& a_{2} \dot{a}_{2}=\frac{A}{2 \pi}\left(a_{3}^{-2}-a_{1}^{-2}\right), \\
& a_{3} \dot{a}_{3}=\frac{A}{2 \pi}\left(a_{1}^{-2}-a_{2}^{-2}\right) .
\end{aligned}
$$

Somando e integrando as equações (3.114), tem-se

$$
a_{1}^{2}+a_{2}^{2}+a_{3}^{2}=\text { constante. }
$$

Por outro lado, multiplicando as equações (3.114) por $a_{1}^{-2}, a_{2}^{-2}$ e $a_{3}^{-2}$ respectivamente, somando e integrando, obtemos:

$$
\ln a_{1}+\ln a_{2}+\ln a_{3}=\text { constante. }
$$


Portanto o sistema (3.112) tem as integrais primeiras (3.115) e (3.116), as quais podem ser escritas na forma :

$$
\begin{aligned}
a_{1}^{2}+a_{2}^{2}+a_{3}^{2} & =c_{2}^{2} \\
a_{1} a_{2} a_{3} & =c_{1}^{3} .
\end{aligned}
$$

Desta maneira, se subtituimos $a_{3}=\frac{c_{1}^{3}}{a_{1} a_{2}}$ no sistema (3.112), obtemos

$$
\begin{aligned}
& \dot{a}_{1}=\frac{1}{2 \pi}\left(\frac{\sin A_{3}}{a_{2}}-\frac{a_{1} a_{2} \sin A_{2}}{c_{1}^{3}}\right), \\
& \dot{a}_{2}=\frac{1}{2 \pi}\left(\frac{a_{1} a_{2} \sin A_{1}}{c_{1}^{3}}-\frac{\sin A_{3}}{a_{1}}\right) .
\end{aligned}
$$

O sistema (3.118) tem o ponto de equilíbrio $a_{0}=\left(c_{1}, c_{1}\right)$, correspondente à configuração de um triângulo equilátero, entendendo-se por configuração à figura geométrica formada pelos vórtices. Da matriz M, do sistema linearizado (3.118),

$$
\left.\mathbf{M}\right|_{a=a_{0}}=\frac{\sqrt{3}}{4 \pi c_{1}^{2}}\left(\begin{array}{cc}
-1 & -2 \\
2 & 1
\end{array}\right)
$$

obtemos os autovalores $\lambda= \pm \frac{3}{4 \pi c_{1}^{2}} i$, portanto o ponto de equilíbrio $a_{0}=\left(c_{1}, c_{1}\right)$ corresponde a um centro.

Ziglin obtem as soluções do sistema de três vórtices numa vizinhança do centro $a_{0}=\left(c_{1}, c_{1}\right)$, as quais são dadas por uma família a um parâmetro de funções periódicas. Escolhendo um parâmetro $\varepsilon$ apropriado para esta família, ele subtitui estas funções periódicas nas duas equações (3.108) de movimento do quarto vórtice, obtendo assim um sistema Hamiltoniano dependendo periódicamente do tempo, a saber

$$
\begin{aligned}
& \frac{\partial \xi}{\partial \tau}=\frac{\partial H}{\partial \eta} \\
& \frac{\partial \eta}{\partial \tau}=-\frac{\partial H}{\partial \xi}
\end{aligned}
$$

onde $H(\xi, \eta, \tau ; \varepsilon)=H_{0}(\xi, \eta)+\varepsilon H_{1}(\xi, \eta, \tau)+\ldots$ 
Quando $\varepsilon=0$, o sistema Hamiltoniano é definido pela função Hamiltoniana $H_{0}$, e o correspondente retrato de fase possui um ponto fixo homoclínico. Para $\varepsilon \neq 0$ é necessário examinar o sistema $(3.120)$ no espaço de fase estendido $\{\xi, \eta, \tau(\bmod 2 \pi)\}$ e considerar a aplicação de Poincaré, definida do plano $\{\tau(\bmod 2 \pi)\}=\tau_{0}$ nele mesmo, dado pelo fluxo de fase. Em geral, se $\varepsilon \neq 0$, a órbita homoclínica do ponto fixo hiperbólico, separa a variedade estável da variedade instável, as quais se interseptam transversalmente no ponto homoclínico. Então o sistema perturbado apresenta comportamento caótico, devido à presença de "horseshoes". A existência de pontos homoclínicos está garantida, já que a função de Melnikov relacionada ao sistema (3.120) tem um zero simple. Para os detalhes, ver [29].

Por outro lado, um outro exemplo de um sistema não integrável é obtido em [18], onde é apresentada uma demonstração completamente analítica da não integrabilidade no problema de quatro vórtices sem massa, no caso particular de dois vórtices fortes e opostos, e dois vórtices fracos e próximos, por meio da integral de Melnikov que é explícitamente calculada por residuos. 


\section{Capítulo 4}

\section{O modelo dos vórtices com massa}

O modelo dos vórtices com massa em $\mathbb{R}^{2}$ pode ser introduzido de duas formas distintas. Numa primeira forma, pode-se definir fisicamente a força que age num vórtice com massa, o qual pode ser considerado como um vórtice onde se encontra uma partícula de impureza, responsável por esta massa. Este procedimento foi feito por Friedrichs, em [11]. Uma outra forma é a utilização da analogia com o modelo das "cargas bidimensionais", discutidas na seção 3.3, agora sem tomar as massas nulas. Estas duas formas de descrever o modelo determinam as mesmas equações do movimento, o que será apresentado na seção 4.1. Como consequência da analogia eletromagnética, obtem-se imediatamente que o modelo resultante é um sistema Hamiltoniano.

Contrariamente ao que acontece no caso dos vórtices sem massa, o sistema de dois vórtices com massa pode ser não integrável. Mostraremos que, para certos valores dos parâmetros, o sistema é não integrável, enquanto para outros, o sistema é integrável (ver [25]). Na seção 4.2, analisaremos o problema de dois vórtices com massa no caso em que a soma de suas intensidades é não nula. Utilizaremos as integrais primeiras do sistema, para reduzí-lo de oito para quatro variáveis. Mostraremos ainda que no caso $\frac{m_{1}}{\Gamma_{1}}=\frac{m_{2}}{\Gamma_{2}}$ o sistema é integrável, já que aparece mais uma integral primeira. Além disso, algumas das órbitas deste sistema ficam próximas às órbitas do sistema com dois vórtices sem massa. 
Finalmente, na seção 4.3, analisaremos o problema de dois vórtices com massa, agora no caso em que a soma de suas intensidades é nula. Novamente utilizaremos as integrais primeiras do sistema para reduzí-lo de oito para quatro variáveis. Entretanto, neste caso, se considerarmos $m_{1}=m_{2}$, obteremos um sistema que não é integrável no sentido de Liouville, para a maioria dos valores dos parâmetros.

\subsection{O modelo dos vórtices com massa como um sis- tema Hamiltoniano}

Vamos considerar a situação em que existem $N$ vórtices movendo-se em $\mathbb{R}^{2}$, sendo que ao $j$-ésimo vórtice está associada não só a intensidade $\Gamma_{j}$ mas também a massa $m_{j}$. De acordo com fórmula de Friedrichs (1966), temos que a força que atua nestes vórtices é:

$$
\mathbf{F}_{j}=\rho \Gamma_{j} \mathbf{e}_{3} \times\left(\dot{\vec{x}}_{j}-\mathbf{u}_{j}\left(\vec{x}_{j}\right)\right), \quad j=1, \ldots, N
$$

onde $\rho$ é a densidade do fluido e

$$
\mathbf{u}_{j}=\frac{1}{\Gamma_{j}}\left(\frac{\partial W}{\partial y_{j}},-\frac{\partial W}{\partial x_{j}}, 0\right)
$$

com $W$ definida em (3.14).

Então, pela segunda lei de Newton, o movimento de $\mathrm{N}$ vórtices com massa é descrito pelas equações:

$$
m_{j} \ddot{\vec{x}}_{j}=\rho \Gamma_{j} \mathbf{e}_{3} \times\left(\dot{\vec{x}}_{j}-\mathbf{u}_{j}\left(\vec{x}_{j}\right)\right), \quad j=1, \ldots, N
$$

Substituindo a equação (4.2) em (4.3) obtemos:

$$
m_{j} \ddot{\vec{x}}_{j}=\rho \Gamma_{j}\left(\mathbf{e}_{3} \times \dot{\vec{x}}_{j}\right)+\nabla_{j}(\rho W), \quad j=1, \ldots, N
$$

Fazendo as identificações $\rho \Gamma_{j} \leftrightarrow-\frac{Q_{j} B}{c}$ e $(\rho W) \leftrightarrow \Phi$ temos que as equações (4.4) são formalmentes iguais a (3.33).

Da observação anterior e dos resultados da seção 3.3 , temos o seguinte teorema: 
Teorema 4.1.1 As equações que descrevem o movimento de $N$ vórtices com massa são determinadas pelo sistema Hamiltoniano $\left(\mathbb{R}^{4 \mathrm{~N}}, w, H\right)$, com função Hamiltoniana $H$ definida por:

$$
H=\sum_{j=1}^{N} \frac{1}{2 m_{j}}\left\|\vec{p}_{j}-\frac{\rho \Gamma_{j}}{2}\left(\vec{x}_{j} \times \mathrm{e}_{3}\right)\right\|^{2}+\rho W,
$$

onde W é a função de Kirchhoff-Routh, definida em (3.14), e forma simplética $w$ definida por:

$$
w=\sum_{j=1}^{N} d p_{x_{j}} \wedge d x_{j}+d p_{y_{j}} \wedge d y_{j} .
$$

Observação 4.1 Note que:

$$
\vec{p}_{j}=m_{j} \dot{\vec{x}}_{j}-\frac{\rho \Gamma_{j}}{2}\left(\mathbf{e}_{3} \times \vec{x}_{j}\right)
$$

Observação 4.2 Analogamente à (3.12), o colchete de Poisson relativo à forma simplética $w$ definida acima é dado por:

$$
\{F, G\}=\sum_{j=1}^{N} \sum_{i=1}^{2}\left(\frac{\partial F}{\partial x_{j_{i}}} \frac{\partial G}{\partial p_{j_{i}}}-\frac{\partial F}{\partial p_{j_{i}}} \frac{\partial G}{\partial x_{j_{i}}}\right)
$$

Devido à invariância do sistema por rotações e translações, verifica-se (utilizandose, por exemplo, o teorema de Noether, ver [3]) que o sistema tem, além da energia, as seguintes integrais primeiras:

$$
\begin{aligned}
\Pi_{x} & =\sum_{j=1}^{N}\left(p_{x_{j}}+\frac{\rho \Gamma_{j}}{2} y_{j}\right), \\
\Pi_{y} & =\sum_{j=1}^{N}\left(p_{y_{j}}-\frac{\rho \Gamma_{j}}{2} x_{j}\right) . \\
J & =\sum_{j=1}^{N}\left(\vec{x}_{j} \times \vec{p}_{j}\right) \cdot \mathrm{e}_{3} .
\end{aligned}
$$

Estas integrais primeiras não estão em involução. De fato, de (4.8), o colchete de Poisson, relativo à forma simplética (4.6), entre as diversas integrais primeiras são:

$$
\left\{\Pi_{x}, \Pi_{y}\right\}=-\rho \sum_{j=1}^{N} \Gamma_{j}
$$




$$
\begin{aligned}
& \left\{\Pi_{x}, J\right\}=\Pi_{y}, \\
& \left\{\Pi_{x}, J\right\}=-\Pi_{x} .
\end{aligned}
$$

No entanto, definindo $\Pi^{2}=\Pi_{x}^{2}+\Pi_{y}^{2}$, verifica-se que:

$$
\left\{\Pi^{2}, J\right\}=0 .
$$

Assim, dos resultados acima, temos a seguinte proposição:

Proposição 4.1.2 O sistema Hamiltoniano $\left(\mathbb{R}^{4 \mathrm{~N}}, \mathrm{w}, \mathrm{H}\right)$, com função hamiltoniana $H$ e forma simplética $w$ definidas em (4.5) e (4.6), respectivamente, tem como integrais primeiras independentes em involução, $\Pi^{2}$ e $J$, além da energia $H$.

Este resultado garante, juntamente com o Teorema de Liouville, a possibilidade de reduzir o sistema de $N$ vórtices com massa, de $4 N$ para $4 N-6$ variáveis. No entanto, esta redução não é suficiente para garantir a integrabilidade nem mesmo de um sistema de dois vótices com massa, como veremos na próxima seção.

\subsection{Exemplo 4: dois vórtices com massa, $\Gamma_{1}+\Gamma_{2} \neq 0$}

\subsubsection{Redução para quatro variáveis}

De (4.5), com $W$ definida em (3.14), temos que a função Hamiltoniana do sistema de dois vórtices com massa em $\mathbb{R}^{2}$ é:

$$
\begin{aligned}
H\left(\vec{x}_{1}, \vec{x}_{2}, \vec{p}_{1}, \vec{p}_{2}\right)= & \frac{1}{2 m_{1}}\left\|\vec{p}_{1}-\frac{\Gamma_{1} \rho}{2}\left(\vec{x}_{1} \times \mathbf{e}_{3}\right)\right\|^{2}+\frac{1}{2 m_{2}}\left\|\vec{p}_{2}-\frac{\Gamma_{2} \rho}{2}\left(\vec{x}_{2} \times \mathbf{e}_{3}\right)\right\|^{2} \\
& +\Phi\left(\left\|\vec{x}_{1}-\vec{x}_{2}\right\|\right)
\end{aligned}
$$

onde $\Phi\left(\left\|\vec{x}_{1}-\vec{x}_{2}\right\|\right)=\rho W\left(\vec{x}_{1}, \vec{x}_{2}\right)=-\rho \frac{\Gamma_{1} \Gamma_{2}}{4 \pi} \ln \left\|\vec{x}_{2}-\vec{x}_{1}\right\|, \vec{x}_{i}=\left(x_{i}, y_{i}\right)$ e $\vec{p}_{i}=\left(p_{x_{i}}, p_{y_{i}}\right)$, $i=1,2$. 
A forma simplética é:

$$
w=d p_{x_{1}} \wedge d x_{1}+d p_{y_{1}} \wedge d y_{1}+d p_{x_{2}} \wedge d x_{2}+d p_{y_{2}} \wedge d y_{2}
$$

De acordo com (4.9), este sistema tem as seguintes integrais primeiras:

$$
\begin{aligned}
\Pi_{x} & =p_{x_{1}}+p_{x_{2}}+\frac{\rho \Gamma_{1}}{2} y_{1}+\frac{\rho \Gamma_{2}}{2} y_{2}, \\
\Pi_{x} & =p_{y_{1}}+p_{y_{2}}-\frac{\rho \Gamma_{1}}{2} x_{1}-\frac{\rho \Gamma_{2}}{2} x_{2}, \\
J & =x_{1} p_{y_{1}}-y_{1} p_{x_{1}}+x_{2} p_{y_{2}}-y_{2} p_{x_{2}} .
\end{aligned}
$$

Sem perda de generalidade, podemos supor $\Gamma_{1}+\Gamma_{2}>0$. Neste caso, guiados pelas simetrias da função Hamiltoniana (4.12), utilizaremos as seguintes coordenadas:

$$
\begin{aligned}
\mathbf{R} & =\frac{\Gamma_{2} \vec{x}_{2}+\Gamma_{1} \vec{x}_{2}}{\Gamma_{1}+\Gamma_{2}} \\
\mathbf{r} & =\vec{x}_{2}-\vec{x}_{1} .
\end{aligned}
$$

Escrevendo $\mathbf{R}$ e $\mathbf{r}$ em coordenadas polares, obtemos:

$$
\begin{aligned}
\mathbf{R} & =R \cos \theta \mathbf{e}_{1}+R \sin \theta \mathbf{e}_{2}, \\
\mathbf{r} & =r \cos (\theta+\phi) \mathbf{e}_{1}+r \sin (\theta+\phi) \mathbf{e}_{2},
\end{aligned}
$$

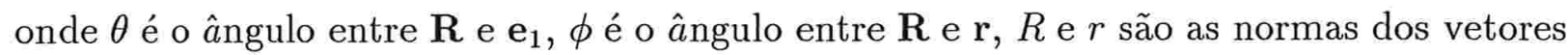
$\mathbf{R}$ e r, respectivamente. De (4.15) e (4.16) tem-se que:

$$
\begin{aligned}
& x_{1}=R \cos \theta-\frac{\Gamma_{2}}{\Gamma_{1}+\Gamma_{2}} r \cos (\theta+\phi), \\
& y_{1}=R \sin \theta-\frac{\Gamma_{2}}{\Gamma_{1}+\Gamma_{2}} r \sin (\theta+\phi), \\
& x_{2}=R \cos \theta+\frac{\Gamma_{1}}{\Gamma_{1}+\Gamma_{2}} r \cos (\theta+\phi), \\
& y_{2}=R \sin \theta+\frac{\Gamma_{1}}{\Gamma_{1}+\Gamma_{2}} r \sin (\theta+\phi) .
\end{aligned}
$$

Para que se obtenha uma transformação canônica, os novos momentos $p_{R}, p_{r}, p_{\theta} \mathrm{e}$ $p_{\phi}$ devem satisfazer:

$$
\vec{q}_{1}=\left(\begin{array}{c}
p_{r} \\
p_{\phi}
\end{array}\right)=\mathbf{M}_{1}\left(-K_{2} \vec{p}_{1}+K_{1} \vec{p}_{2}\right)
$$




$$
\vec{q}_{2}=\left(\begin{array}{c}
p_{R} \\
p_{\theta}-p_{\phi}
\end{array}\right)=\mathbf{M}_{2}\left(\vec{p}_{1}+\vec{p}_{2}\right)
$$

onde $K_{i}=\frac{\Gamma_{i}}{\Gamma_{1}+\Gamma_{2}}, \mathrm{i}=1,2 \mathrm{e}$

$$
\mathbf{M}_{1}=\left(\begin{array}{cc}
\cos (\theta+\phi) & \sin (\theta+\phi) \\
r \sin (\theta+\phi) & r \cos (\theta+\phi)
\end{array}\right), \quad \mathbf{M}_{2}=\left(\begin{array}{cc}
\cos \theta & \sin \theta \\
-R \sin \theta & R \cos \theta
\end{array}\right),
$$

de onde obtem-se:

$$
\begin{aligned}
& \vec{p}_{1}=K_{1} \mathbf{M}_{2}^{-1} \vec{q}_{2}-\mathbf{M}_{1}^{-1} \vec{q}_{1}, \\
& \vec{p}_{2}=K_{2} \mathbf{M}_{2}^{-1} \vec{q}_{2}-\mathbf{M}_{1}^{-1} \vec{q}_{1} .
\end{aligned}
$$

Se notamos que:

$$
\begin{aligned}
& \mathbf{M}_{1}^{-1}=\frac{1}{r}\left(\begin{array}{cc}
r \cos (\theta+\phi) & -\sin (\theta+\phi) \\
r \sin (\theta+\phi) & \cos (\theta+\phi)
\end{array}\right)=T_{-\theta} T_{-\phi} \mathbf{M}_{r}^{-1}, \\
& \mathbf{M}_{2}^{-1}=\frac{1}{R}\left(\begin{array}{cc}
R \cos \theta & R \sin \theta \\
\sin \theta & \cos \theta
\end{array}\right)=T_{-\theta} \mathbf{M}_{R}^{-1},
\end{aligned}
$$

com

$$
T_{\theta}=\left(\begin{array}{cc}
\cos \theta & \sin \theta \\
-\sin \theta & \cos \theta
\end{array}\right), \quad \mathbf{M}_{R}=\left(\begin{array}{cc}
1 & 0 \\
0 & R
\end{array}\right), \quad \mathbf{M}_{r}=\left(\begin{array}{cc}
1 & 0 \\
0 & r
\end{array}\right),
$$

podemos escrever (4.19) na forma

$$
\begin{aligned}
& \vec{p}_{1}=T_{-\theta}\left(\frac{\Gamma_{1}}{\Gamma_{1}+\Gamma_{2}} \mathbf{M}_{R}^{-1}\left(\begin{array}{c}
p_{R} \\
p_{\theta}-p_{\phi}
\end{array}\right)-T_{-\phi} \mathbf{M}_{r}^{-1}\left(\begin{array}{c}
p_{r} \\
p_{\phi}
\end{array}\right)\right), \\
& \vec{p}_{2}=T_{-\theta}\left(\frac{\Gamma_{2}}{\Gamma_{1}+\Gamma_{2}} \mathbf{M}_{R}^{-1}\left(\begin{array}{c}
p_{R} \\
p_{\theta}-p_{\phi}
\end{array}\right)+T_{-\phi} \mathbf{M}_{r}^{-1}\left(\begin{array}{c}
p_{r} \\
p_{\phi}
\end{array}\right)\right) .
\end{aligned}
$$

A função Hamiltoniana (4.12), nas novas variáveis canônicas, é dada por:

$$
H=\left(\frac{m_{1}+m_{2}}{2 m_{1} m_{2}}\right)\left(p_{r}^{2}+\frac{p_{\phi}^{2}}{r^{2}}\right)+\frac{\rho^{2}}{8}\left(\frac{\Gamma_{1} \Gamma_{2}}{\Gamma_{1}+\Gamma_{2}}\right)^{2}\left(\frac{m_{1}+m_{2}}{m_{1} m_{2}}\right) r^{2}+\Phi(r)+
$$




$$
\begin{aligned}
& +\frac{1}{2}\left(\frac{\Gamma_{1}^{2}}{m_{1}}+\frac{\Gamma_{2}^{2}}{m_{2}}\right)\left(\frac{1}{\Gamma_{1}+\Gamma_{2}}\right)^{2}\left(p_{R}^{2}+\left(\frac{p_{\theta}-p_{\phi}}{R}\right)^{2}+\frac{\rho^{2}}{4} R^{2}\left(\Gamma_{1}+\Gamma_{2}\right)^{2}\right)+ \\
& +\left(\frac{\Gamma_{2}}{m_{2}}-\frac{\Gamma_{1}}{m_{1}}\right)\left(\frac{1}{\Gamma_{1}+\Gamma_{2}}\right) f+\frac{\rho}{2}\left(\frac{\Gamma_{2}}{m_{2}}-\frac{\Gamma_{1}}{m_{1}}\right)\left(\frac{\Gamma_{1} \Gamma_{2}}{\left(\Gamma_{1}+\Gamma_{2}\right)^{2}}\right) g+ \\
& +\frac{\rho}{2}\left(\frac{\Gamma_{2}}{m_{2}}-\frac{\Gamma_{1}}{m_{1}}\right)\left(\frac{\Gamma_{1}-\Gamma_{2}}{\Gamma_{1}+\Gamma_{2}}\right) p_{\phi}+\frac{\rho}{2}\left(\frac{\Gamma_{1}^{2}}{m_{1}}+\frac{\Gamma_{2}^{2}}{m_{2}}\right)\left(\frac{1}{\Gamma_{1}+\Gamma_{2}}\right) p_{\theta}
\end{aligned}
$$

onde $f$ e $g$ são dadas por:

$$
\begin{aligned}
f= & \left(p_{R} p_{r}+\left(\frac{p_{\theta}-p_{\phi}}{R r}\right) p_{\phi}\right) \cos \phi \\
& +\left(-\frac{p_{R} p_{\phi}}{r}+\left(\frac{p_{\theta}-p_{\phi}}{R}\right) p_{r}\right) \sin \phi+\frac{\rho}{2} R\left(p_{r} \sin \phi+\frac{p_{\phi}}{r} \cos \phi\right)\left(\Gamma_{1}+\Gamma_{2}\right),
\end{aligned}
$$

e

$$
g=\frac{\rho}{2}\left(\Gamma_{1}+\Gamma_{2}\right) R r \cos \phi-r\left(p_{R} \sin \phi-\left(\frac{p_{\theta}-p_{\phi}}{R}\right) \cos \phi\right) .
$$

Note que a depêndencia da Hamiltoniana com $\phi$ está toda nas funções $f$ e $g$. Como a transformção é canônica, a forma simplética nestas variáveis é:

$$
w=d p_{R} \wedge d R+d p_{r} \wedge d r+d p_{\theta} \wedge d \theta+d p_{\phi} \wedge d \phi
$$

e as integrais primeiras (4.14) são:

$$
\begin{aligned}
{\left[\begin{array}{l}
\Pi_{x} \\
\Pi_{y}
\end{array}\right] } & =T_{-\theta}\left[\begin{array}{c}
p_{R} \\
\frac{p_{\theta}-p_{\phi}}{R}-\frac{\rho}{2}\left(\Gamma_{1}+\Gamma_{2}\right) R
\end{array}\right], \\
J & =p_{\theta},
\end{aligned}
$$

com $T_{\theta}$ definido em (4.20).

Notemos que $p_{\theta}$ é integral primeira e então a Hamiltoniana (4.22), que não depende de $\theta$, é reduzida a seis variáveis. Assim, conhecida a solução do sistema $\left(\mathbb{R}^{6}, \tilde{w}, \tilde{H}\right)$, com função Hamiltoniana

$$
\widetilde{H}\left(R . r, \Phi, p_{R}, p_{r}, p_{\Phi}\right)=H\left(R, r, \Phi, p_{R}, p_{r}, p_{\Phi} ; p_{\theta}\right)
$$


e forma simplética

$$
\tilde{w}=d p_{R} \wedge d R+d p_{r} \wedge d r+d p_{\phi} \wedge d \phi
$$

podemos determinar o valor de $\theta$, através de integração:

$$
\theta=\theta\left(t_{0}\right)+\int_{t_{0}}^{t} \frac{\partial H}{\partial p_{\theta}} d t
$$

Para reduzir ainda mais o número de variáveis na Hamiltoniana (4.22), utilizaremos a integral primeira $\Pi^{2}$. A partir de qualquer condição inicial, com uma translação conveniente, é possivel fazer $\Pi^{2}=0$, desde que $\Gamma_{1}+\Gamma_{2} \neq 0$. Portanto, sem perda de generalidade, podemos supor $\Pi^{2}=0$. Assim da relação (4.26) temos:

$$
\begin{array}{rlr}
p_{R} & =0 & \left(\text { donde } \dot{p}_{R}=0\right), \\
\frac{p_{\theta}-p_{\phi}}{R}-\frac{\rho}{2}\left(\Gamma_{1}+\Gamma_{2}\right) R & =0 & \text { ou } R=\sqrt{\frac{2}{\rho\left(\Gamma_{1}+\Gamma_{2}\right)}\left(p_{\theta}-p_{\phi}\right) .}
\end{array}
$$

Substituindo as relações (4.30) e (4.31) diretamente na Hamiltoniana (4.22), eliminamos as variáveis $p_{R}$ e $R$, obtendo assim o sistema $\left(H^{\prime}, w^{\prime}\right)$, que também é Hamiltoniano, com função Hamiltoniana $H^{\prime}$, dada por

$$
H^{\prime}\left(r, \phi, p_{r}, p_{\phi} ; p_{\theta}\right)=H\left(R\left(p_{\phi} ; p_{\theta}\right), r, \phi, P_{R}=0, p_{r}, p_{\phi} ; p_{\theta}\right)
$$

com $R=R\left(p_{\phi} ; p_{\theta}\right)$, definido em (4.31) e forma simplética:

$$
w^{\prime}=d p_{r} \wedge d r+d p_{\phi} \wedge d \phi .
$$

De fato, verifica-se que as equações que determinam o movimento para este sistema Hamiltoniano, são equivalentes às do sistema anterior, isto é:

$$
\begin{gathered}
\dot{r}=\frac{\partial H^{\prime}}{\partial p_{r}}\left(r, \phi, p_{r}, p_{\phi} ; p_{\theta}\right)=\frac{\partial H}{\partial p_{r}}\left(R\left(p_{\phi} ; p_{\theta}\right), r, P_{R}=0, p_{r}, p_{\phi} ; p_{\theta}\right) \\
\dot{\phi}=\frac{\partial H^{\prime}}{\partial p_{\phi}}\left(r, \phi, p_{r}, p_{\phi} ; p_{\theta}\right)=\left(\frac{\partial H}{\partial p_{\phi}}\left(R\left(p_{\phi} ; p_{\theta}\right), r, \phi, P_{R}=0, p_{r}, p_{\phi} ; p_{\theta}\right)\right)_{\mathrm{R}=\mathrm{constante}}+ \\
+\frac{\partial H}{\partial R}\left(R\left(p_{\phi} ; p_{\theta}\right), r, \phi, P_{R}=0, p_{r}, p_{\phi} ; p_{\theta}\right) \frac{\partial R}{\partial p_{\phi}}=
\end{gathered}
$$




$$
\begin{aligned}
& =\frac{\partial H}{\partial p_{\phi}}\left(R\left(p_{\phi} ; p_{\theta}\right), r, \phi, P_{R}=0, p_{r}, p_{\phi} ; p_{\theta}\right), \\
\dot{p_{r}} & =-\frac{\partial H^{\prime}}{\partial r}\left(r, \phi, p_{r}, p_{\phi} ; p_{\theta}\right)=-\frac{\partial H}{\partial r}\left(R\left(p_{\phi} ; p_{\theta}\right), r, \phi, P_{R}=0, p_{r}, p_{\phi} ; p_{\theta}\right), \\
\dot{p_{\phi}} & =-\frac{\partial H^{\prime}}{\partial \phi}\left(r, \phi, p_{r}, p_{\phi} ; p_{\theta}\right)=-\frac{\partial H}{\partial r}\left(R\left(p_{\phi} ; p_{\theta}\right), r, \phi, P_{R}=0, p_{r}, p_{\phi} ; p_{\theta}\right),
\end{aligned}
$$

já que, da condição (4.30) tem-se:

$$
\dot{p_{R}}=-\frac{\partial H}{\partial R}\left(R\left(p_{\phi} ; p_{\theta}\right), r, \phi, P_{R}=0, p_{r}, p_{\phi} ; p_{\theta}\right)=0 .
$$

A função Hamiltoniana reduzida $H^{\prime}$, definida em (4.32), nas novas variáveis é

$$
\begin{aligned}
H^{\prime} & =\left(\frac{m_{1}+m_{2}}{2 m_{1} m_{2}}\right)\left[p_{r}^{2}+\left(\frac{p_{\phi}}{r}\right)^{2}+\frac{\rho^{2}}{4}\left(\frac{\Gamma_{1} \Gamma_{2}}{\Gamma_{1}+\Gamma_{2}}\right)^{2} r^{2}\right]+ \\
& +\frac{\rho}{\Gamma_{1}+\Gamma_{2}}\left(\frac{\Gamma_{1}^{2}}{m_{1}}+\frac{\Gamma_{2}^{2}}{m_{2}}\right)\left(p_{\theta}-p_{\phi}\right)+\Phi(r)+ \\
& +\left(\frac{\Gamma_{2}}{m_{2}}-\frac{\Gamma_{1}}{m_{1}}\right) \frac{1}{\sqrt{\Gamma_{1}+\Gamma_{2}}} \sqrt{2 \rho\left(p_{\theta}-p_{\phi}\right)}\left(\frac{p_{\phi}}{r} \cos \phi+p_{r} \sin \phi+\frac{\rho}{2} \frac{\Gamma_{1} \Gamma_{2}}{\Gamma_{1}+\Gamma_{2}} r \cos \phi\right) .
\end{aligned}
$$

Resumindo os resultados anteriores, temos a seguinte proposição:

Proposição 4.2.1 O sistema Hamiltoniano $\left(\mathbb{R}^{8}, w, H\right)$, com $H$ e w definidas em (4.22) $e(4.25)$ respectivamente, com $\Gamma_{1}+\Gamma_{2} \neq 0$, é reduzido ao sistema Hamiltoniano $\left(\mathbb{R}^{4}, w^{\prime}\right.$, $\left.H^{\prime}\right)$, dependendo da integral primeira $p_{\theta}$ como parâmetro, com $w^{\prime}$ definidas em (4.33) e $H^{\prime}$ definida em (4.36).

Note que a Hamiltoniana $H^{\prime}$ pode ser reescrita como:

$$
\begin{aligned}
H^{\prime}= & \frac{1}{2 \mu}\left(p_{r}^{2}+\frac{p_{\phi}^{2}}{r^{2}}+\gamma^{2} r^{2}+2 \gamma p_{\phi}\right)+\xi\left(p_{\theta}-p_{\phi}\right)+\boldsymbol{\Phi}(r)+ \\
& +\vartheta \sqrt{2 \rho\left(p_{\theta}-p_{\phi}\right)}\left(\frac{p_{\phi}}{r} \cos \phi+p_{r} \sin \phi+\gamma r \cos \phi\right)= \\
= & \frac{1}{2 \mu}\left(\vec{p}-\gamma\left(\vec{r} \times \mathbf{e}_{3}\right)\right)^{2}+\xi\left(p_{\theta}-p_{\phi}\right)+\boldsymbol{\Phi}(r)+ \\
& +\vartheta \sqrt{2 \rho\left(p_{\theta}-p_{\phi}\right)}\left(\vec{p}-\gamma\left(\vec{r} \times \mathbf{e}_{3}\right)\right) \cdot \mathbf{e}_{2}
\end{aligned}
$$


onde $\vec{r}=r \mathbf{e}_{r}, \vec{p}=p_{r} \mathbf{e}_{r}+\frac{p_{\phi}}{r} \mathbf{e}_{\phi}, \mathbf{e}_{r}$ e $\mathbf{e}_{\phi}$ são os vetores polares unitários usuais, e onde foram definidas as constantes:

$$
\begin{aligned}
\mu & =\frac{m_{1} m_{2}}{m_{1}+m_{2}} \\
\gamma & =\frac{\rho}{2} \frac{\Gamma_{1} \Gamma_{2}}{\Gamma_{1}+\Gamma_{2}} \\
\xi & =\frac{\rho}{\Gamma_{1}+\Gamma_{2}}\left(\frac{\Gamma_{1}^{2}}{m_{1}}+\frac{\Gamma_{2}^{2}}{m_{2}}\right) \\
\vartheta & =\left(\frac{\Gamma_{2}}{m_{2}}-\frac{\Gamma_{1}}{m_{1}}\right) \frac{1}{\sqrt{\Gamma_{1}+\Gamma_{2}}}
\end{aligned}
$$

\subsubsection{Um caso integrável : $\frac{m_{1}}{\Gamma_{1}}=\frac{m_{2}}{\Gamma_{2}}$}

Nesta subseção descreveremos a dinâmica de um sistema de dois vótices com massa, que é integrável no sentido de Liouville, no caso particular em que ambos vórtices têm o mesmo raio massa/vorticidade:

$$
\frac{m_{1}}{\Gamma_{1}}=\frac{m_{2}}{\Gamma_{2}}=\beta, \quad \beta>0
$$

Assim, sob a hipótese (4.39), podemos ver que alguns termos da Hamiltoniana (4.22) são eliminados. A Hamiltoniana não depende mais de $\phi$ e portanto obtemos que $p_{\phi}$ é outra constante de movimento. Além disso:

$$
\dot{\theta}=\left(\frac{\Gamma_{1}^{2}}{m_{1}}+\frac{\Gamma_{2}^{2}}{m_{2}}\right)\left(\frac{1}{\Gamma_{1}+\Gamma_{2}}\right)\left(\frac{p_{\theta}-p_{\phi}}{R^{2}\left(\Gamma_{1}+\Gamma_{2}\right)}+\frac{\rho}{2}\right)
$$

Utilizando agora a relação (4.31), obtemos:

$$
\begin{aligned}
\dot{\theta} & =\xi=\text { constante } \\
R & =\sqrt{\frac{2}{\rho\left(\Gamma_{1}+\Gamma_{2}\right)}\left(p_{\theta}-p_{\phi}\right)}=\text { constante }
\end{aligned}
$$

com $\xi$ definido em (4.38).

Isto implica que, a menos de uma constante imposta para obter $\Pi^{2}=0$, o vetor $\mathbf{R}$, definido em (4.15), tem módulo constante e roda com velocidade constante. Então, é 
suficiente estudar a dinâmica de r, definido por (4.16), determinada pela função Hamiltoniana (4.37). Se notamos, a partir de (4.39), que $\vartheta=0$ e $\xi=(2 \gamma) / \mu$, podemos escrever a função Hamiltoniana (4.37) na forma:

$$
H^{\prime}=\frac{1}{2 \mu} p_{r}^{2}+\mathbf{U}(r)
$$

onde

$$
\begin{gathered}
\mathbf{U}=\frac{1}{2 \mu}\left(\frac{p_{\phi}}{r}-\gamma r\right)^{2}-\alpha \ln r, \\
\alpha=\rho \frac{\Gamma_{1} \Gamma_{2}}{2 \pi} .
\end{gathered}
$$

Assim, conhecida a solução do sistema Hamiltoniano $\left(H_{1}, w_{1}\right)$, com função Hamiltoniana

$$
H_{1}\left(r, p_{r}\right)=H^{\prime}\left(r, p_{r} ; p_{\phi}\right)
$$

e forma simplética

$$
w_{1}=d p_{r} \wedge d r
$$

é possível obter a solução do sistema original através de quadraturas. De fato, temos:

$$
\begin{aligned}
\phi(t) & =\phi(0)+\int_{0}^{t} \frac{\partial H^{\prime}}{\partial p_{\phi}} d s= \\
& =\phi(0)+\frac{1}{\mu} \int_{0}^{t}\left(\frac{p_{\phi}}{r^{2}(s)}-\gamma\right) d s
\end{aligned}
$$

com

$$
p_{\phi}=\mu(\mathbf{r} \times \dot{\mathbf{r}}) \cdot \mathbf{e}_{3}+\gamma r^{2}
$$

onde $r(s)$ representa a solução do sistema definido por (4.45) e (4.46).

As equações do movimento associado à função Hamiltoniana (4.43) são:

$$
\begin{aligned}
\dot{r} & =\frac{p_{r}}{\mu} \\
\dot{p_{r}} & =-\frac{\partial \mathbf{U}}{\partial r}=\frac{1}{\mu}\left(\frac{p_{\phi}^{2}}{r^{3}}-\gamma^{2} r\right)+\frac{\alpha}{r} .
\end{aligned}
$$


A função potencial U admite um único ponto critico, o qual é um ponto de mínimo, $r_{*}>0$, dado por:

$$
r_{*}^{2}=\frac{\mu \alpha+\sqrt{(\mu \alpha)^{2}+\left(2 \gamma p_{\phi}\right)^{2}}}{2 \gamma^{2}}
$$

Portanto, sendo $r_{*}$ um ponto mínimo do potencial $\mathbf{U},\left(r_{*}, 0\right)$, é um centro do sistema (4.49).

\subsubsection{Estabilidade}

É claro que os modelos dos vórtices com massa e dos vórtices sem massa são totalmente distintos enquanto sistemas Hamiltonianos. As dimensões do espaço de fase de um sistema com $N$ vórtices são $4 N$ e $2 N$, respectivamente, e parte das variáveis tipo "posição"no modelo com massa passará a ser variável tipo "momento"no modelo dos vórtices sem massa. Entretanto, em situações muito particulares, foi possível mostrar (ver [25]) que órbitas dos dois modelos ficam próximas, num certo sentido. Mais precisamente, pode-se comparar a órbita circular obtida no modelo de dois vórtices sem massa, com órbitas perto do centro obtido acima, para massas suficientemente pequenas. Se considerarmos como condição inicial para as equações (4.49) um ponto sobre a solução (3.43), obteremos uma órbita particular do sistema dos vórtices com massa que está próxima da órbita (3.43), no sentido de que sua projeção $r(t)$ no espaço de configurações do sistema sem massa satisfaz:

$$
\sup _{t \in R}\left\|r(t)-r_{0}\right\| \rightarrow 0, \text { quando } \beta \rightarrow 0 .
$$

A sequinte proposição foi mostrada em [25]:

Proposição 4.2.2 Dada uma órbita $\gamma_{1}$, do sistema de dois vórtices sem massa com intensidade total não nula, existe uma órbita $\gamma_{2}$, do sistema de dois vórtices com as mesma intensidade, mas com massas $m_{i}=\Gamma_{i} \beta, i=1,2$, tal que a projeção da órbita $\gamma_{2}$ sobre o espaço de configuração tende a $\gamma_{1}$, quando as massas tendem a zero, sob as mesmas condições iniciais. 
Entretanto, mesmo neste caso foi possível mostrar que as duas soluções não ficam próximas quando olhadas com função do tempo.

\subsection{Exemplo 5: dois vórtices com massa, $\Gamma_{1}+\Gamma_{2}=0$}

\subsubsection{Redução para quatro variáveis}

Nesta subsecão, reduziremos o sistema de dois vórtice com massa de oito para quatro variáveis, no caso da soma das intensidade ser nula, isto é, $-\Gamma_{1}=\Gamma_{2}=\Gamma$. Neste caso, a Hamiltoniana (4.5) escreve-se:

$$
H=\frac{1}{2 m_{1}}\left\|\vec{p}_{1}-\frac{\Gamma \rho}{2}\left(\vec{x}_{1} \times \mathbf{e}_{3}\right)^{2}\right\|+\frac{1}{2 m_{2}}\left\|\vec{p}_{2}+\frac{\Gamma \rho}{2}\left(\vec{x}_{2} \times \mathbf{e}_{3}\right)^{2}\right\|+\Phi\left(\left\|\vec{x}_{2}-\vec{x}_{1}\right\|\right),
$$

onde $\Phi\left(\left\|\vec{x}_{2}-\vec{x}_{1}\right\|\right)=\frac{\rho \Gamma^{2}}{4 \pi} \ln \left\|\vec{x}_{2}-\vec{x}_{1}\right\|$, e a forma simplética é:

$$
w=d \vec{p}_{1} \wedge d \vec{x}_{1}+d \vec{p}_{2} \wedge d \vec{x}_{2}
$$

De acordo com (4.14), este sistema tem as integrais primeiras:

$$
\begin{aligned}
\Pi_{x} & =p_{x_{1}}+p_{x_{2}}+\frac{\rho}{2} \Gamma\left(y_{1}-y_{2}\right) \\
\Pi_{y} & =p_{y_{1}}+p_{y_{2}}-\frac{\rho}{2} \Gamma\left(x_{1}-x_{2}\right) \\
J & =x_{1} p_{y_{1}}-y_{1} p_{x_{1}}+x_{2} p_{y_{2}}-y_{2} p_{x_{2}} .
\end{aligned}
$$

Já que $\Gamma_{1}+\Gamma_{2}=0$, a partir de (4.10), vemos que as integrais primeiras $\Pi_{x}$ e $\Pi_{y}$ estão em involução, isto é, $\left\{\Pi_{x}, \Pi_{y}\right\}=0$.

Consideremos as sequintes mudanças de coordenadas:

$$
\begin{aligned}
\mathbf{R} & =\frac{m_{1} \vec{x}_{1}+m_{2} \vec{x}_{2}}{m_{1}+m_{2}} \\
\mathbf{r} & =\vec{x}_{2}-\vec{x}_{1} .
\end{aligned}
$$


As relações em (4.54), implicam:

$$
\begin{aligned}
& \vec{x}_{1}=\mathbf{R}-\frac{m_{2}}{m_{1}+m_{2}} \mathbf{r}, \\
& \vec{x}_{2}=\mathbf{R}+\frac{m_{1}}{m_{1}+m_{2}} \mathbf{r} .
\end{aligned}
$$

Para que a transformação seja canônica, escolheremos:

$$
\begin{aligned}
\vec{p}_{\mathbf{R}}^{\prime} & =\vec{p}_{1}+\vec{p}_{2} \\
\vec{p}_{\mathbf{r}}^{\prime} & =\frac{m_{1}}{m_{1}+m_{2}} \vec{p}_{2}-\frac{m_{2}}{m_{1}+m_{2}} \vec{p}_{1} .
\end{aligned}
$$

A partir de (4.56), temos que:

$$
\begin{aligned}
& \overrightarrow{p_{1}}=\frac{m_{1}}{m_{1}+m_{2}} \overrightarrow{p_{\mathbf{R}}}-{\overrightarrow{p_{\mathbf{r}}}}^{\prime} \\
& \vec{p}_{2}=\frac{m_{2}}{m_{1}+m_{2}}{\overrightarrow{p_{\mathbf{R}}}}^{\prime}+{\overrightarrow{p_{\mathbf{r}}}}^{\prime} .
\end{aligned}
$$

A forma simplética se escreve:

$$
w=d \vec{p}_{1} \wedge d \vec{x}_{1}+d \vec{p}_{2} \wedge d \vec{x}_{2}=d{\overrightarrow{p_{\mathbf{R}}}}^{\prime} \wedge d \mathbf{R}+d{\overrightarrow{p_{\mathbf{r}}}}^{\prime} \wedge d \mathbf{r}
$$

e a Hamiltoniana (4.51), nas novas coordenadas ${\overrightarrow{p_{\mathbf{R}}}}^{\prime}, \vec{p}_{\mathbf{r}}{ }^{\prime}, \mathbf{R}, \mathbf{r}$, é:

$$
\begin{aligned}
H_{1}= & \frac{1}{2}\left(\frac{m_{1}+m_{2}}{m_{1} m_{2}}\right){\overrightarrow{p_{\mathbf{r}}}}^{\prime 2}+\frac{1}{2}\left(\frac{1}{m_{1}+m_{2}}\right){\overrightarrow{\mathbf{p}_{\mathbf{R}}}}^{\prime 2} \\
& +\frac{1}{2}\left(\frac{\Gamma \rho}{2}\right)^{2}\left(\frac{m_{1}+m_{2}}{m_{1} m_{2}}\right) \mathbf{R}^{2}+\frac{1}{2}\left(\frac{\Gamma \rho}{2}\right)^{2}\left(\frac{m_{1}^{3}+m_{2}^{3}}{m_{1} m_{2}\left(m_{1}+m_{2}\right)}\right) \mathbf{r}^{2} \\
& +\left(\frac{\Gamma \rho}{2}\right)\left(\frac{m_{1}+m_{2}}{m_{1} m_{2}}\right){\overrightarrow{p_{\mathbf{r}}}}^{\prime} \cdot\left(\mathbf{R} \times \mathbf{e}_{3}\right) \\
& +\left(\frac{\Gamma \rho}{2}\right)\left(\frac{1}{m_{1}+m_{2}}\right){\overrightarrow{p_{\mathbf{R}}}}^{\prime} \cdot\left(\mathbf{r} \times \mathbf{e}_{3}\right) \\
& +\left(\frac{\Gamma \rho}{2}\right)\left(\frac{m_{1}^{2}-m_{2}^{2}}{m_{1} m_{2}\left(m_{1}+m_{2}\right)}\right)\left({\overrightarrow{p_{\mathbf{r}}}}^{\prime}+\frac{\Gamma \rho}{2}\left(\mathbf{R} \times \mathbf{e}_{3}\right)\right) \cdot\left(\mathbf{r} \times \mathbf{e}_{3}\right)+\Phi(\mathbf{r}) .
\end{aligned}
$$

As integrais primeiras, de acordo com (4.53) são:

$$
\begin{aligned}
\Pi & =\overrightarrow{p_{\mathbf{R}}}-\frac{\Gamma \rho}{2}\left(\mathbf{r} \times \mathbf{e}_{3}\right), \\
J & ={\overrightarrow{p_{\mathbf{R}}}}^{\prime} \cdot\left(\mathbf{R} \times \mathbf{e}_{3}\right)+{\overrightarrow{p_{\mathbf{r}}}}^{\prime} \cdot\left(\mathbf{r} \times \mathbf{e}_{3}\right) .
\end{aligned}
$$


Guiados pela relação (4.60), definimos a nova transformação canônica:

$$
\begin{aligned}
\vec{p}_{\mathbf{R}} & =\overrightarrow{p_{\mathbf{R}}^{\prime}}-\frac{\Gamma \rho}{2}\left(\mathbf{r} \times \mathbf{e}_{3}\right), \\
\vec{p}_{\mathbf{r}} & =\overrightarrow{p_{\mathbf{r}}^{\prime}}+\frac{\Gamma \rho}{2}\left(\mathbf{R} \times \mathbf{e}_{3}\right),
\end{aligned}
$$

obtendo assim a função Hamiltoniana (4.59), nas coordenadas $\vec{p}_{\mathbf{R}}, \vec{p}_{\mathbf{r}}, \mathbf{R}, \mathbf{r}$, onde $\vec{p}_{\mathbf{R}}, \vec{p}_{\mathbf{r}}$ são os novos momentos e $\mathbf{R}, \mathbf{r}$ são as antigas coordenadas de posição, dada por:

$$
\begin{aligned}
\widetilde{H}= & \frac{1}{2}\left(\frac{m_{1}+m_{2}}{m_{1} m_{2}}\right)\left(\vec{p}_{\mathbf{r}}{ }^{2}-\Gamma \rho\left(\frac{m_{2}-m_{1}}{m_{1}+m_{2}}\right) \vec{p}_{\mathbf{r}} \cdot\left(\mathbf{r} \times \mathbf{e}_{3}\right)\right. \\
& \left.+\left(\frac{\Gamma \rho}{2}\right)^{2}\left(\frac{m_{2}-m_{1}}{m_{1}+m_{2}}\right)^{2}\left(\mathbf{r} \times \mathbf{e}_{3}\right)^{2}\right) \\
+ & \Phi(\mathbf{r})+\frac{1}{2} \frac{1}{m_{1}+m_{2}}\left(\vec{p}_{\mathbf{R}}{ }^{2}+2 \Gamma \rho \vec{p}_{\mathbf{R}} \cdot\left(\mathbf{r} \times \mathbf{e}_{3}\right)+4\left(\frac{\Gamma \rho}{2}\right)^{2}\left(\mathbf{r} \times \mathbf{e}_{3}\right)^{2}\right)
\end{aligned}
$$

e a forma simplética

$$
w=d \vec{p}_{\mathbf{R}} \wedge d \mathbf{R}+d \vec{p}_{\mathbf{r}} \wedge d \mathbf{r}
$$

Notemos que a Hamiltoniana (4.62) não depende da coordenada $\mathbf{R}$, o que implica que $\vec{p}_{\mathbf{R}}$ é constante de movimento. De fato, temos que $\vec{p}_{\mathbf{R}}$, definido em (4.61), coincide com a integral primeira $\Pi$. Mostramos portanto:

Proposição 4.3.1 O sistema Hamiltoniano $\left(\mathbb{R}^{8}, w, H\right)$, com $H$ e w definidos em (4.51) $e$ (4.52) respectivamente, quando $\Gamma_{1}+\Gamma_{2}=0$, é reduzido ao sistema Hamiltoniano $\left(\mathbb{R}^{4}, w, H\right)$, nas variáveis $\left(\mathbf{r}, \vec{p}_{\mathbf{r}}\right)$, com $H$ dado por

$$
\begin{aligned}
H= & \frac{1}{2 \mu}\left(\vec{p}_{\mathbf{r}}-\frac{\eta}{2}\left(\mathbf{r} \times \mathbf{e}_{3}\right)\right)+\Phi(\mathbf{r}) \\
& +\frac{1}{2}\left(\frac{\Gamma \rho}{\sqrt{m_{1}+m_{2}}}\right)^{2}\left(\left(\mathbf{e}_{3} \times \frac{\vec{p}_{\mathbf{R}}}{\Gamma \rho}\right)+\mathbf{r}\right)^{2},
\end{aligned}
$$

onde

$$
\eta=\Gamma \rho \frac{m_{1}-m_{2}}{m_{1}+m_{2}}
$$

e $\mu$ é definido em (4.38). 


\subsubsection{Um caso não integrável: $m_{1}=m_{2}=m$}

Em geral, é muito difícil mostrar que um sistema é integrável sem encontrar suas integrais primeiras. Mas é possível provar, em alguns casos, que um sistema é não integrável mediante o estudo de algumas propriedades locais, perto de uma solução particular do sistema. Vamos considerar um sistema Hamiltoniano numa variedade de dimensão 4. Um dos poucos métodos analíticos para mostrar a não integrabilidade, em casos específicos, é o método de Melnikov, que utilizamos na seção 3.4 para mostrar a não integrabilidade de um sistema de quatro vórtices sem massa. Um resultado fundamental, devido a Poincaré, é que se um sistema tem uma órbita periódica, tipo sela, e as variedades estável e instável desta órbita interceptam-se transversalmente, então o sistema é não integrável. Neste caso, o sistema tem uma órbita homoclínica transversa. A presença de órbitas homoclínicas transversas implica a existência de conjuntos invariantes (topologicamente "horseshoe"), com uma dinâmica muito complicada e esta complexidade não permite a presença de outra integral primeira independente da função Hamiltoniana.

Um outro resultado nesta direção, devido a Lerman (ver [24],[25]), implica a não integrabilidade do sistema de dois vórtices com massas $m_{1}=m_{2}=m$ e com intensidade total nula $\left(-\Gamma_{1}=\Gamma_{2}=\Gamma\right)$. Este sistema tem duas propriedades importantes que permitem a aplicação do teorema de Lerman :

- O sistema tem um ponto de equilíbrio tipo sela-centro, isto é, a matriz do sistema linearizado, em torno do ponto de equilíbrio, tem um par de autovalores reais e um par de autovalores imaginários puros.

- Há uma órbita homoclínica, associada a este ponto de equilíbrio.

Nesta situação, pode-se mostrar que o sistema linearizado em torno deste ponto de equilíbrio tem órbitas periódicas, tipo sela, em todos os níveis de energia. A existência de uma órbita homoclínica, sugere que as variedades estável e instável destas órbitas periódicas podem interceptar-se. Por argumentos genéricos, esta intersecção pode ocorrer 
transversalmente. Seria de se esperar, levando em conta o resultado de Poincaré, que um sistema nestas condições fosse não integrável. O teorema de Lerman formaliza estas idéias, mas impõe ainda outra hipótese além das citadas acima, para que a propriedade genérica que garante o intersecção trasversal seja satisfeita. Em [24], C. Ragazzo reescreve esta hipótese numa forma que é de aplicação mais simples. Em [25], esta hipótese é testada: a demostração de não integrabilidade seguirá da verificação de que um certo operador de "monodronomia", associado à óbita homoclínica, que é representado por uma equação diferencial linear, não autônoma de segunda ordem, satisfaz uma certa condição genérica. Foi possível mostrar numericamente, que este operador de monodromia satisfaz, de fato, para a maioria dos valores de $\Gamma$ e $m$, a condição genérica. Isto sugere fortemente que o sistema de dois vórtices com massa, com $-\Gamma_{1}=\Gamma_{2}=\Gamma$, não é integrável, no sentido de Liouville.

Por outro lado, é possível mostrar (ver [13]), mediante uma versão do teorema de KAM, a existência de um conjunto de condições iniciais do sistema, com medida de Lebesgue não nula, para as quais o movimento é quase periódico. Podemos concluir então, dos resultados precedentes, que no mesmo sistema Hamiltonianos coexistem órbitas quase periódicas e órbitas com comportamento caótico. 


\section{Capítulo 5}

\section{Modelo misto dos vórtices}

O propósito deste capítulo é introduzir o modelo misto dos vórtices, ou seja, o modelo no qual alguns dos vórtices tem massa e outros não. $\mathrm{Na}$ secão 5.1 , introduziremos as definições básicas e alguns resultados relativos a variedades de Poisson. Veremos, em particular, que uma variedade simplética sempre será uma variedade de Poisson, e que os campos Hamiltonianos definidos em uma variedade simplética e os campos Hamiltonianos definidos a partir da estrutura de Poisson correspondente, para a mesma função Hamiltoniana, coincidem. Na seção 5.2, apresentamos, de maneira intrínsica, o formalismo de Dirac (ver [4]), para sistema Hamiltoniano com vínculos. Este formalismo nos permite, sob certas condições, definir uma estrutura de Poisson natural numa subvariedade $\mathrm{N}$ de uma variedade simplética $\mathrm{M}$. Se $N$ for obtido como a imagem inversa de um valor regular de um função diferenciável de $M$ com valores em $\mathbb{R}^{\mathrm{s}}$ estas condições estarão satisfeitas sempre que uma certa matriz for inversível. Na seção 5.3, aplicamos o formalismo de Dirac para obter o modelo misto dos vórtices a partir do modelo dos vórtices com massa (estudado no capitulo 3), restrito a uma subvariedade do seu espaço de fase. Esta subvariedade é escolhida de forma que a Hamiltoniana associada ao sistema dos vórtices com massa não se torne singular quando fazemos algumas das massas tenderem para zero. $\mathrm{O}$ modelo obtido também é Hamiltoniano e apresenta duas integrais primeiras em involução 
independentes da Hamiltoniana.

Finalmente, na secão 5.4, estudaremos o sistema com um vórtice com massa e outro sem massa, nos casos em que a soma das intensidades é nula ou não. A redução para um grau de liberdade é bastante diferente nos dois casos, o que implica que os retratos de fase dos dois sistemas também são bastante diferentes. Embora o modelo misto com dois vórtices seja sempre integrável, pode-se mostrar (ver [12]) que para certos valores dos parâmetros, o sistema misto com três vórtices é não integrável.

\subsection{Variedades de Poisson}

Nesta secão, introduziremos o conceito de variedade de Poisson, bem como algumas outras definições e resultados que serão utilizados na próxima seção. Mostraremos ainda que estas definições generalizam as noções correspondentes em variedades simpléticas.

Definição 5.1 Uma estrutura de Poisson numa variedade $M$ é determinada por uma aplicação bilinear $\{\cdot, \cdot\}: C^{\infty}(M) \times C^{\infty}(M) \longrightarrow C^{\infty}(M)$, chamada colchete de Poisson, satisfazendo as seguintes propriedades:

- Antissimetria

$$
\{F, H\}=-\{H, F\}, \quad \forall F, H \in C^{\infty}(M),
$$

- Identidade de Jacobi

$$
\{\{F, H\}, G\}+\{\{H, G\}+F\}+\{\{G, F\}, H\}=0,
$$

- Regra de Leibnitz

$$
\{F, H \cdot G\}=\{F, H\} \cdot G+H \cdot\{F, G\}
$$

onde ". "denota a multiplicação ordinária de funções a valores reais e $C^{\infty}(M)=$ $C^{\infty}(M, \mathbb{R})$. 
Definição 5.2 Uma variedade $M$ dotada de uma estrutura de Poisson é denominada variedade de Poisson.

Definição 5.3 Se N, M são variedades de Poisson, uma aplicação de Poisson é uma aplicação $\phi \in C^{\infty}(N, M)$, a qual preserva o colchete de Poisson, isto é,

$$
\{F \circ \phi, H \circ \phi\}_{N}=\{F, H\}_{M} \circ \phi, \quad \forall F, H \in C^{\infty}(M) .
$$

Definição 5.4 Uma subvariedade $N \subset M$ é uma subvariedade de Poisson se a imersão $\phi: N \longrightarrow M$ é uma aplicacão de Poisson.

Observação 5.1 O significado desta definição é que, para qualquer par de funções $\widetilde{F}$, $\widetilde{G} \in C^{\infty}(M)$ tais que $\left.\widetilde{F}\right|_{N}=\widetilde{F} \circ \phi=F \quad$ e $\left.\widetilde{G}\right|_{N}=\widetilde{G} \circ \phi=G$, tem-se:

$$
\left.\{\widetilde{F}, \widetilde{G}\}_{M}\right|_{N}=\{\widetilde{F}, \widetilde{G}\}_{M} \circ \phi=\{F, G\}_{N}, \quad \text { com } F, G \in C^{\infty}(N)
$$

isto é, o colchete de Poisson entre funções definidas em $M$, restrito a $N$, coincide com o colchete de Poisson de funções em $N$.

Observação 5.2 Se notarmos que, dada uma função $H \in C^{\infty}(M)$, a aplicação

$$
\{H, \cdot\}: C^{\infty}(M) \longrightarrow C^{\infty}(M)
$$

é linear e satisfaz a regra de Leibnitz, temos então que a aplicação (5.2) define uma derivação e portanto determina um campo vetorial diferenciável sobre $M$.

Temos, portanto, a seguinte definição fundamental:

Definição 5.5 Seja $M$ uma variedade de Poisson e $H \in C^{\infty}(M)$. O campo vetorial Hamiltoniano associado a $H$, é o único campo vetorial $X_{H}$ sobre $M$ satisfazendo

$$
X_{H}(F)=\{F, H\}=-\{H, F\}, \quad \forall F \in C^{\infty}(M) .
$$


Observação 5.3 Notemos que, fixado $F \in C^{\infty}(M)$, tem-se:

$$
\{G, F\}(p)=X_{F}(p) G(p)=d G(p) X_{F}(p),
$$

onde $d G$ é a diferencial de $G$. Portanto, $\{F, G\}(p)$ depende só de $d G(p)$, isto é, se $G_{1}$ e $G_{2}$ são tais que $d G_{1}(p)=d G_{2}(p)$ então $\left\{F, G_{1}\right\}(p)=\left\{F, G_{2}\right\}(p)$. Da mesma forma, fixando $G \in C^{\infty}(M),\{F, G\}$ só depende de $d F(p)$, quando $F$ varia.

Portanto, do fato da aplicação $F \in C^{\infty}(M) \longrightarrow d F(p) \in T_{p}^{*} M$ ser sobrejetora, da bilinearidade e antissimetria da aplicação $\{\cdot, \cdot\}$, temos que, para todo $p \in M$, existe una forma diferenciável bilinear $W$ e antissimétrica no espaço $T^{*} M$, tal que para todo $F, G$ em $C^{\infty}(M)$

$$
W_{p}(d F(p), d G(p))=\{F, G\}(p) .
$$

O tensor $W$ é chamado o tensor de Poisson.

A partir das observações acima, temos o seguinte resultado:

Proposição 5.1.1 Dada uma variedade de Poisson $(M,\{\cdot, \cdot\})$, está bem definido o tensor diferenciável $W: T^{*} M \times T^{*} M \rightarrow \mathbb{R}$, bilinear e antissimétrico, que para cada $p \in M$ vale:

$$
W_{p}(d F(p), d G(p))=\{F, G\}(p), \quad \forall F, G \in C^{\infty}(M) .
$$

Em coordenadas locais $\left(x_{1}, \cdots, x_{n}\right)$ em $U \subset M$, a estrutura de Poisson é dada por:

$$
\{F, G\}=\sum_{i, j=1}^{N} v_{i j} \frac{\partial F}{\partial x_{i}} \frac{\partial G}{\partial x_{j}}
$$

onde as funções $v_{i j}$ são determinadas por:

$$
v_{i j}=\left\{x_{i}, x_{j}\right\}=W\left(d x_{i}, d x_{j}\right), 1 \leq i, j \leq n .
$$

Da antissimetria e da identidade de Jacobi, temos que as funções $v_{i j}$ satisfazem as seguintes identidades:

$$
\begin{aligned}
v_{i j}+v_{j i} & =0 \\
\sum_{l=1}^{n} v_{l j} \frac{\partial v_{i k}}{\partial x_{l}}+v_{l i} \frac{\partial v_{k j}}{\partial x_{l}}+v_{k l} \frac{\partial v_{j i}}{\partial x_{l}} & =0 .
\end{aligned}
$$


Observação 5.4 Notemos que, reciprocamente, se são dadas $v_{i j}, 1 \leq i, j \leq n$ em $C^{\infty}(x(U))$ satisfazendo (5.9), elas definem localmente um colchete de Poisson. Para uma demonstração desse fato, ver [28].

No caso de uma variedade de Poisson $\left(M^{n}, W\right)$ satisfazer que o posto de $W_{p}$ é $n$, para todo $p$ em $M$, pode-se demonstrar que esta variedade é, na realidade, uma variedade simplética. Para que possamos explicitar a relação entre as duas estruturas, observemos que se $(M, W)$ é uma variedade de Poisson, dado um $\nu \in T_{p}^{*} M$, fixo, a aplicação

$$
\widetilde{W}_{p}(\nu)=W_{p}(\nu, \cdot): T_{p}^{*} M \rightarrow \mathbf{R}
$$

está no dual do espaço $T_{p}^{*} M$, isto é, $\widetilde{W}_{p}(\nu) \in\left(T_{p}^{*} M\right)^{*}=T_{p} M$, já que a dimensão de $M$ é finita. Portanto, está bem definida a aplicação linear $\widetilde{W}_{p}: T_{p}^{*} M \rightarrow T_{p} M$ tal que :

$$
\left\langle\widetilde{W}_{p}(\nu), \eta\right\rangle=W_{p}(\nu, \eta) \quad \forall \nu, \eta \in T_{p}^{*} M
$$

onde $\langle\cdot, \cdot\rangle$ é o emparelhamento de $T_{p} M$ e $T_{p}^{*} M$. É imediato que, quando posto $\left(W_{p}\right)=$ $n, \forall p \in M$, a aplicação linear $\widetilde{W}_{p}$ é um isomorfismo.

Analogamente, se $w$ é uma forma simplética em $M$, podemos definir um isomorfismo $\widetilde{w}_{p}: T_{p} M \rightarrow T_{p}^{*} M$ tal que

$$
\left\langle\widetilde{w}_{p}(v), u\right\rangle=w_{p}(v, u) \quad \forall v, u \in T_{p} M
$$

Podemos então enunciar a seguinte proposição:

Proposição 5.1.2 Seja $\left(M^{n}, W\right)$ uma variedade de Poisson, com posto de $W_{p}$ igual a $n$, para todo $p$ em $M$. Então, se definirmos $\tilde{w}_{p}=\left[\tilde{W}_{p}\right]^{-1}$, temos que w é uma forma simplética em $M$, ou seja, é uma 2-forma fechada e não degenerada. Reciprocamente, dada uma forma simplética w em $M$, se definirmos $\widetilde{W}_{p}=\left[\widetilde{w}_{p}\right]^{-1}$, temos que $\widetilde{W}_{p}$ é um tensor de Poisson em $M$.

Demostração: ver [28]. 
Observação 5.5 As estruturas de Poisson e simplética relacionadas como na proposição acima são ditas compatíveis.

Lembremos que o campo vetorial Hamiltoniano, associado a $H$ numa variedade simplética $(M, w)$, é o único campo vetorial $X_{H_{1}}$ definido, para cada $p \in M$, por (ver definição $3.2)$ :

$$
w_{p}\left(\mu, X_{H_{1}}(p)\right)=d H_{p}(\mu) \quad \forall \mu \in T_{p} M
$$

Por outro lado, se olhamos esta variedade simplética como uma variedade de Poisson $(M, W)$ (com estrutura simplética e de Poisson compatíveis), o campo hamiltoniano $X_{H_{2}}$ asociado a $H$, de acordo com as equações (5.3) e (5.5), é o único campo vetorial definido, para cada $p$ em $M$, por:

$$
X_{H_{2}}(G)_{p}=\{G, H\}(p)=W_{p}\left(d G_{p}, d H_{p}\right) \quad \forall G \in C^{\infty}(M) .
$$

Para que estas definicões sejam compatíveis, precisamos mostrar que $X_{H_{1}}$ e $X_{H_{2}}$ associados à mesma função Hamiltoniana coincidem.

A partir de (5.10) e (5.13), tem-se que:

$$
\left\langle\widetilde{W}_{p}\left(d H_{p}\right), \eta\right\rangle=W_{p}\left(d H_{p}, \eta\right)=\{H, G\}(p)=-X_{H_{2}}(p)(\eta)=-\left\langle X_{H_{2}}(p), \eta\right\rangle \quad \forall \eta \in T_{p}^{*} M
$$

onde $G$ é qualquer função diferenciável tal que $d G(p)=\eta$. De 5.14 tem-se que:

$$
\widetilde{W}_{p}\left(d H_{p}\right)=-X_{H_{2}}(p)
$$

Por outro lado, para todo $v \in T_{p} M$ tem-se que:

$$
\left\langle\widetilde{w}_{p}\left(X_{H_{1}}(p)\right), v\right\rangle=w_{p}\left(X_{H_{1}}(p), v\right)=-w\left(v, X_{H_{1}}(p)\right)=-d H(v)=-\langle d H, v\rangle
$$

donde

$$
\widetilde{w}_{p}\left(X_{H_{2}}(p)\right)=-d H_{p}
$$


Logo,

$$
X_{H_{1}}=(\widetilde{W} \circ \widetilde{w})\left(X_{H_{1}}\right)=\widetilde{W}\left(\widetilde{w}\left(X_{H_{1}}\right)\right)=\widetilde{W}(-d H)=X_{H_{2}},
$$

que é o que queríamos demonstrar. Concluímos que, de fato, a definição de variedade de Poìsson generaliza bem a definição de variedade simplética.

Para finalizarmos esta seção, observemos que o conceito de sistema Hamiltoniano é imediatamente generalizado para variedades de Poisson, já que o campo Hamiltoniano $X_{H}$ continua definido (ver a definição 3.4 ).

\subsection{Método de Dirac}

Nesta seção, mostraremos que, sob certas condições, é possível munir de uma estrutura de Poisson (ou simplética) uma subvariedade $\mathrm{N}$ de uma variedade de Poisson (ou simplética) M. Mostraremos ainda que, neste caso, se $N$ é uma subvariedade invariante de um sistema Hamiltoniano, podemos definir um sistema Hamiltoniano retrito a $N$, de forma que as trajetórias deste sistema Hamiltoniano restrito coincidam com as trajetórias do sistema Hamiltoniano original que estejam em $N$.

Seja $M$ uma variedade de Poisson, com tensor de Poisson $W$, e $N$ uma subvariedade de $M$. Suponha que exista um operador linear $L: C^{\infty}(M) \rightarrow C^{\infty}(M)$ tal que:

$$
\left.L(H)\right|_{N}=\left.H\right|_{N}=\vec{H}, \quad \forall H \in C^{\infty}(M) .
$$

Este operador $L$ induz um outro operador $L_{*}$, que age sobre os campos vetoriais Hamiltonianos em $M$, do sequinte modo:

$$
L_{*}\left(X_{H}\right)=X_{L(H)}, \quad \forall H \in C^{\infty}(M) .
$$

Suponha que $L_{*}$ satisfaz as seguintes propriedades:

$$
\left(L_{*} X_{H}\right)(p) \quad \in T_{p} N, \quad \forall p \in N, \quad \forall H \in C^{\infty}(M),
$$


e, para todo campo vetorial Hamiltoniano $X_{H}$ sobre $M$, tangente a $N$, se verifica:

$$
L_{*} X_{H}(p)=X_{H}(p), \quad \forall H \in C^{\infty}(M), \text { tal que } \mathrm{X}_{\mathrm{H}}(\mathrm{p}) \in \mathrm{T}_{\mathrm{p}} \mathrm{N}, \quad \forall \mathrm{p} \in \mathrm{N} \text {. }
$$

Com auxílio dos operadores $L$ e $L_{*}$ podemos definir, de maneira natural, uma estrutura de Poisson em $N$, conforme o teorema abaixo:

Teorema 5.2.1 Seja $M$ uma variedade de Poisson, com colchete de Poisson $\{\cdot, \cdot\}$ e $N$ uma subvariedade de $M$. Suponha dado um operador $L: C^{\infty}(M) \rightarrow C^{\infty}(M)$ satisfazendo (5.18), (5.20) e (5.21). Então

(i) $N$ pode ser munida de uma estrutura de Poisson, com colchete de Poisson $\{\cdot, \cdot\}^{\prime}$ definido por:

$$
\{F, G\}^{\prime}:=\left.\{L(\widetilde{F}), L(\widetilde{G})\}\right|_{N}, \quad \forall F, G \in C^{\infty}(N),
$$

onde $\widetilde{F}$ e $\widetilde{G}$ são extensões quaisquer de $F$ e G, respectivamente.

(ii) Se $M$ é uma variedade simplética com forma simplética $w$ e $i^{*} w$ é não degenerada, então $\left(N, i^{*} w\right)$ é uma variedade simplética, com forma simplética $\bar{w}=i^{*} w$, compatível com a estrutura de Poisson acima.

Demonstração: (i) Precisamos mostrar inicialmente que o colchete $\{\cdot, \cdot\}^{\prime}$ está bem definido, isto é, não depende da escolha das extensões. Comecemos por demonstrar que dados $\widetilde{G}_{1}$ e $\widetilde{G}_{2}$ extensões quaisquer de $G$, tem-se:

$$
\left.\left\{L(\widetilde{F}), \widetilde{G}_{1}\right\}\right|_{N}=\left.\left\{L(\widetilde{F}), \widetilde{G}_{2}\right\}\right|_{N} .
$$

Dado que $L(\widetilde{F}) \in C^{\infty}(M)$, tem-se pela equação (5.3), que existe um único campo vetorial $X_{L(\widetilde{F})}$, sobre $M$, tal que:

$$
\left.\{L(\widetilde{F}), R\}\right|_{N}=\left[X_{L(\widetilde{F})}(R)\right]_{N}, \forall R \in C^{\infty}(M) .
$$

Como $X_{L(\widetilde{F})}$ é tangente a $N$, isto é, $X_{L(\widetilde{F})}(p) \in T_{p} N, \forall p \in N$, então $X_{L(\widetilde{F})}(p)$ é o vetor tangente a alguma curva $\alpha: I \rightarrow N \operatorname{com} \alpha(0)=p$ :

$$
X_{L(\tilde{F})}(p)=\left.\frac{d \alpha(t)}{d t}\right|_{t=0}
$$


Assim, de (5.23) tem-se:

$$
\left\{L(\widetilde{F}), \widetilde{G}_{i}\right\}=-X_{L(\widetilde{F})}\left(\widetilde{G}_{i}\right)=-\frac{d}{d t}\left(\left.\tilde{G}_{i}(\alpha(t))\right|_{t=0}=-\left.\frac{d}{d t} G(\alpha(t))\right|_{t=0}, i=1,2,\right.
$$

o que demonstra (5.22). Da antissimetria de $\{\cdot, \cdot\}$, é imediato que se $\widetilde{F}_{1}$ e $\widetilde{F}_{2}$ são extensões quaisquer de $F$,

$$
\left.\left\{\widetilde{F}_{1}, L(\widetilde{G})\right\}\right|_{N}=\left.\left\{\widetilde{F}_{2}, L(\widetilde{G})\right\}\right|_{N}
$$

Mas, se $\widetilde{F}_{1}$ e $\widetilde{F}_{2}$ são extensões de $F$, e $\widetilde{G}_{1}$ e $\widetilde{G}_{2}$ são extensões de $G$, então $L\left(\widetilde{F}_{1}\right)$ e $L\left(\widetilde{F}_{2}\right)$ também são extensões de $F$, e $L\left(\widetilde{G}_{1}\right)$ e $L\left(\widetilde{G}_{2}\right)$ também são extensões de $G$. Portanto:

$$
\left.\left\{L\left(\widetilde{F}_{1}\right), L\left(\widetilde{G}_{1}\right)\right\}\right|_{N}=\left.\left\{L\left(\widetilde{F_{1}}\right), L\left(\widetilde{G}_{2}\right)\right\}\right|_{N}=\left.\left\{L\left(\widetilde{F}_{2}\right), L\left(\widetilde{G}_{2}\right)\right\}\right|_{N} .
$$

Assim, de (5.25) conclui-se que

$$
\{F, G\}^{\prime}:=\left.\{L(\widetilde{F}), L(\widetilde{G})\}\right|_{N}, \quad \forall F, G \in C^{\infty}(N)
$$

está bem definida, ou seja, não depende das extensões $\widetilde{F}$ e $\widetilde{G}$ escolhidas. Mostramos ainda que:

$$
\{F, G\}^{\prime}(p)=\{L(\widetilde{F}), L(\widetilde{G})\}(p)=-X_{L(\widetilde{F})}(p)\left(L(\widetilde{G})(p)=-X_{L(\widetilde{F})}(p)(G)(p),\right.
$$

ou seja, o campo vetorial Hamiltoniano associado a $\{\cdot, \cdot\}^{\prime}$ é:

$$
Z_{F}=\left.X_{L(\tilde{F})}\right|_{N}
$$

Resta verificar que $\{\cdot, \cdot\}^{\prime}$ é um colchete de Poisson em $N$, ou seja, satisfaz as propriedades da definicão (5.1). Pela definição (5.26), é imediato que $\{\cdot, \cdot\}^{\prime}$ é bilinear e antissimétrico. Para demonstrarmos a regra de Leibnitz, notemos que se $\widetilde{F}, \widetilde{G}$, e $\widetilde{H}$ são extensões de $F, G$ e $H$ a $M$, respectivamente, então $\widetilde{G} \widetilde{H}$ é uma extensão $\widetilde{G H}$ de $G H$ e portanto,

$$
\{F, G H\}^{\prime}=\left.\{L(\widetilde{F}), L(\widetilde{G H})\}\right|_{N}=
$$




$$
\begin{aligned}
& =\left.\{L(\widetilde{F}), L(\widetilde{G} \tilde{H})\}\right|_{N}= \\
& =\left.\{L(\widetilde{F}), \widetilde{G} \widetilde{H}\}\right|_{N}= \\
& =[\widetilde{G}\{L(\widetilde{F}), \widetilde{H}\}+\{L(\widetilde{F}), \widetilde{H}\} \widetilde{H}]_{N}= \\
& =\left.G\{L(\widetilde{F}), \widetilde{H}\}\right|_{N}+\left.\{L(\widetilde{F}), \widetilde{G}\}\right|_{N} H= \\
& =G\{F, H\}^{\prime}+\{F, G\}^{\prime} H .
\end{aligned}
$$

Por último, mostraremos que $\{\cdot, \cdot\}^{\prime}$ satisfaz a identidade de Jacobi. Previamente, notemos que uma extensão de

$$
W=\{G, H\}^{\prime}=\left.\{L(\widetilde{G}), L(\widetilde{H})\}\right|_{N} \in C^{\infty}(N)
$$

a $M$ é

$$
\widetilde{W}=\{L(\widetilde{G}), L(\widetilde{H})\} \in C^{\infty}(M) .
$$

Portanto,

$$
\{F, W\}^{\prime}=\left.\{L(\widetilde{F}), L(\widetilde{W})\}\right|_{N}=\left.\{L(\widetilde{F}), L(\{L(\widetilde{G}), L(\widetilde{H})\})\}\right|_{N}=\left.\{L(\widetilde{F}),\{L(\widetilde{G}), L(\widetilde{H})\}\}\right|_{N}
$$

Assim, da observacão acima, temos que:

$$
\begin{gathered}
\left\{F,\{G, H\}^{\prime}\right\}^{\prime}+\left\{G,\{H, F\}^{\prime}\right\}^{\prime}+\left\{H,\{F, G\}^{\prime}\right\}^{\prime}= \\
\left.\{L(\widetilde{F}), L(\{L(\widetilde{G}), L(\widetilde{H})\})\}\right|_{N}+\left.\{L(\widetilde{G}), L(\{L(\widetilde{H}), L(\widetilde{F})\})\}\right|_{N}+ \\
\left.\{L(\widetilde{H}), L(\{L(\widetilde{F}), L(\widetilde{G})\})\}\right|_{N}= \\
\left.\{L(\widetilde{F}),\{L(\widetilde{G}), L(\widetilde{H})\})\}\left.\right|_{N}+\{L(\widetilde{G}),\{L(\widetilde{H}), L(\widetilde{F})\})\right\}\left.\right|_{N}+ \\
\{L(\widetilde{H}),\{L(\widetilde{F}), L(\widetilde{G})\})\}\left.\right|_{N}= \\
[\{L(\widetilde{F}),\{L(\widetilde{G}), L(\widetilde{H})\})\}+\{L(\widetilde{F}),\{L(\widetilde{G}), L(\widetilde{H})\})\}+ \\
\{L(\widetilde{F}),\{L(\widetilde{G}), L(\widetilde{H})\})\}]_{N}=0
\end{gathered}
$$

já que $\{\cdot, \cdot\}$ é um colchete de Poisson, e portanto satisfaz a identidade de Jacobi. 
(ii)Dada a inclusão $i: N \rightarrow M$, tem-se a aplicacão $i_{*}$ (ver [1]), o pull-back de $i$,

$$
\begin{array}{cc}
i^{*}: \Omega^{2}(M) & \rightarrow \Omega^{2}(N) \\
w & \rightarrow i^{*} w=\bar{w}
\end{array}
$$

onde $\Omega^{2}(M)$ é o espaço vetorial das 2 -formas diferenciais sobre $M$, o qual é definido, para cada $p \in N$, por:

$$
\left(i^{*} w\right)_{p}\left(z_{1}, z_{2}\right)=w_{i(p)}\left(i_{* p} z_{1}, i_{* p} z_{2}\right), \quad \forall z_{1}, z_{2} \in T_{p} N
$$

onde $i_{* p}: T_{p} N \rightarrow T_{i(p)} M$ é a diferencial de $i$.

Dada uma variedade simplética $(M, w)$, pelas propriedades do "pull-back" tem-se que

$$
d\left(i^{*} w\right)=i^{*} d w=0
$$

ou seja, $i^{*} w$ é fechada. Por sua definição, é claro que $i^{*} w$ é bilinear e antissimétrica. Uma condicão necessária (mas não suficiente), para que $i^{*} w$ seja não degenerada, é que a dimensão de $N$ seja par. Para que $\left(N, i^{*} w\right)$ seja uma variedade simplética basta exigir que $i^{*} w$ seja não degenerada .

Para mostrarmos que as duas estruturas são compatíveis, note que:

$$
\begin{gathered}
\{H, G\}^{\prime}=\left.\{L(\tilde{H}), L(\tilde{G})\}\right|_{N}=W\left(d(L(\tilde{H})),\left.d(L(\tilde{G}))\right|_{N}=\right. \\
=\left.w\left(X_{L(\tilde{H})}, X_{L(\tilde{G})}\right)\right|_{N}=i^{*} w\left(\left.X_{L(\tilde{H})}\right|_{N},\left.X_{L(\tilde{H})}\right|_{N}\right),
\end{gathered}
$$

já que, por hipótese, $\left.X_{L(\tilde{H})}\right|_{N},\left.X_{L(\tilde{G})}\right|_{N}$ são campos vetoriais em $N$, para todas as fuções $H, G \in C^{\infty}(N)$. Levando em conta a equação $(5.28)$, mostramos que $Z_{H}=\left.X_{L(\tilde{H})}\right|_{N}$ é o campo Hamiltoniano correspondente tanto à estrutura de Poisson definida em (i) quanto à estrutura simplética definida em (ii), que é o que queríamos demonstrar.

Podemos agora enunciar o corolário que nos permite a redução de sistemas Hamiltonianos a subvariedades invariantes de $\mathrm{M}$ :

Corolario 5.2.1 Seja $M$ uma variedade de Poisson (ou, simplética) que satisfaça as hipóteses do teorema 5.2.1(i) (ou (ii)). Se $H: M \rightarrow \mathbb{R}$ é uma Hamiltoniana que admite 
$N$ como variedade invariante, então a restricão $\bar{H}$ de $H$ a $N$, define, juntamente com a estrutura de Poisson (ou simplética) do teorema 5.2.1, um sistema Hamiltoniano cujas órbitas coincidem com as órbitas do sistema Hamiltoniano definido por $H$ que estejam contidas em $N$.

Demonstração: Basta observar que as trajetórias do sistema Hamiltoniano em $N$ são as curvas integrais de $Z_{\bar{H}}=\left.X_{L(H)}\right|_{N}$, ou seja são as curvas $\gamma: I \rightarrow N$ que satisfazem:

$$
\dot{\gamma}(t)=Z_{\bar{H}}(\gamma(t))=X_{L(H)}(\gamma(t))
$$

e que portanto são trajetórias do sistema Hamiltoniano definido em $M$, com Hamiltoniana $L(H)$. Mas, por hipótese, $N$ é invariante por $H$, donde $X_{H}(p) \in T_{p} N, \forall p \in N$. Então, pela hipótese $(5.21),\left.X_{L(H)}\right|_{N}=\left.X_{H}\right|_{N}$. Logo, as trajetórias de $(N, \bar{H})$ são as trajetórias de $(M, H)$, que estejam em $N$. Reciprocamente, toda trajetória do sistema Hamiltoniano em $M$, que esta contido em $N$, satisfaz a relação acima, e portanto é uma trajetória do sistema Hamiltoniano em $N$.

Agora, aplicaremos o teorema anterior, para fazer o processo de restrição de um sistema Hamiltoniano a uma subvariedade. Seja $(M,\{\cdot, \cdot\})$ uma variedade de Poisson e seja $N$ uma subvariedade, definida por:

$$
\left\{p \in M \text { tal que } \Phi_{1}(p)=\Phi_{2}(p)=\cdots=\Phi_{s}(p)=0\right\}
$$

onde $\Phi_{i} \in C^{\infty}(M), i=1,2, \cdots, s$, e $d \Phi_{1}(p), d \Phi_{2}(p), \cdots, d \Phi_{s}(p)$ são linearmente independentes, para todo $p \in N$. Suponhamos ainda que a matriz $C(p)=\left(c_{i j}(p)\right)_{1 \leq i, j \leq s}$, com $c_{i j}(p)=\left\{\Phi_{i}, \Phi_{j}\right\}(p)$ seja inversível, para todo $p \in M$. Então, para cada função $F \in C^{\infty}(M)$ e para cada ponto $p \in M$, é possível resolver o sistema de equaçoẽs lineares abaixo:

$$
\left\{\Phi_{i}, F\right\}(p)+\sum_{k=1}^{s} \lambda_{k}(p)\left\{\Phi_{i}, \Phi_{k}\right\}(p)=0 .
$$

Podemos definir $L: C^{\infty}(M) \rightarrow C^{\infty}(M)$ por:

$$
L(F)=F+\sum_{k=1}^{s} \lambda_{k} \Phi_{k},
$$


onde os coeficientes $\lambda_{k}$, determinados por (5.33), dependem linearmente de $F$.

Podemos agora mostrar que:

Proposição 5.2.1 O operador L, definido em (5.34), satisfaz as hipóteses do teorema 5.2.1.

Demonstração: É claro, da definição de $L$, que (5.18) está satisfeita. Para mostrar que (5.20) também está satisfeita, note que $v \in T_{p} N$ se e sómente se $d \Phi_{i}(p)(v)=0, i=$ $1,2, \cdots, s$. Mas, por (5.3), (5.1) e (5.33),

$$
\begin{gathered}
d \Phi_{i}(p)\left(X_{L(F)}\right)(p)=\left\{\Phi_{i}, L(F)\right\}(p)= \\
=\left\{\Phi_{i}, F\right\}(p)+\sum_{k=1}^{s}\left(\lambda_{k}(p)\left\{\Phi_{i}, \Phi_{k}\right\}(p)+\Phi_{k}(p)\left\{\Phi_{i}, \lambda_{k}\right\}\right)=0,
\end{gathered}
$$

para todo $p \in N$, o que mostra (5.20).

Para mostrarmos que (5.21) é válida, consideremos $F \in C^{\infty}(M)$ tal que $X_{F}(p) \in$ $T_{p} N, \quad \forall p \in N$. Então $d \Phi_{i}(p)\left(X_{F}\right)(p)=\left\{\Phi_{i}, F\right\}(p)=0$, donde $\lambda_{i}(p)=0, \forall p \in N, i=$ $1,2, \cdots, s$. Neste caso, de (5.1) e (5.3), para toda $G \in C^{\infty}(M)$, temos que:

$$
\begin{gathered}
\left.X_{L(F)}(G)(p)=\left\{G, F+\sum_{k=1}^{s} \lambda_{k} \Phi_{k}\right\}(p)\right\}= \\
=\{G, F\}(p)+\sum_{k=1}^{s}\left(\lambda_{k}(p)\left\{G, \Phi_{k}\right\}(p)+\Phi_{k}(p)\left\{G, \lambda_{k}\right\}\right)=X_{F}(G)(p),
\end{gathered}
$$

para todo $p \in N$. Mostramos portanto que $X_{L(F)}(p)=X_{F}(p), \forall p \in N$, o que completa nossa demonstração

$\mathrm{O}$ colchete de Poisson induzido em $N$ é dado por:

$$
\{F, G\}^{\prime}=\left.\{L(\widetilde{F}), \widetilde{G}\}\right|_{N}=\left[\{\widetilde{F}, \widetilde{G}\}+\sum_{k=1}^{s} \lambda_{k}\left\{\Phi_{k}, \widetilde{G}\right\}\right]_{N}
$$


Sejam $a_{i j}(p), 1 \leq i, j \leq s$ os elementos de $C^{-1}(p)$. Então, $\lambda_{k}=-\sum_{j=1}^{s} a_{k j}\left\{\Phi_{j}, \widetilde{F}\right\}(p)$, donde

$$
\{F, G\}^{\prime}=\left[\{\widetilde{F}, \widetilde{G}\}-\sum_{k, j=1}^{s} a_{k j}\left\{\Phi_{j}, \widetilde{F}\right\}\left\{\Phi_{k}, \widetilde{G}\right\}\right]_{N}
$$

onde $\widetilde{F}$ e $\widetilde{G}$ são extensões a $M$, das função $F$ e $G$ respectivamente.

\subsection{O modelo misto dos vórtices}

Nesta seção, construiremos um modelo em que um dos $N$ vórtices não tenha massa. Para tanto, vamos partir do sistema de $N$ vórtices com massa, que é o sistema Hamiltoniano $\left(\mathbb{R}^{4 N}, w, H\right)$, com Hamiltoniana $H$ e forma simplética w definidas em (4.5) e (4.6), respectivamente. Note que não podemos, na Hamiltoniana $H$ tomar $m_{1}=0$, pois $m_{1}$ aparece no denominador. Para controlar este problema, definimos uma subvariedade $N$ de $\mathbb{R}^{4 N}$, de tal forma que exista o limite de $\left.H\right|_{N}$ quando $m_{1} \rightarrow 0$, e do formalismo de Dirac, apresentado na seção anterior, definiremos uma estructura de Poisson. O corolário (5.2.1) sugere que consideremos nesta variedade de Poisson um sistema Hamiltoniano, com Hamiltoniana $\left.H\right|_{N}$ quando $m_{j}=0$.

Seja $N \subset \mathbb{R}^{4 N}$ a subvariedade de $\mathbb{R}^{4 N}$ definida por:

$$
N=\left\{\left(\vec{x}, \vec{p}_{x}\right) \in \mathbb{R}^{4 N} \text { tq. } \Phi_{x}=\Phi_{y}=0\right\}
$$

onde

$$
\begin{gathered}
\Phi_{x}=p_{x_{1}}-\frac{\Gamma_{1} \rho}{2} y_{1}=0 \\
\Phi_{y}=p_{y_{1}}+\frac{\Gamma_{1} \rho}{2} x_{1}=0 .
\end{gathered}
$$

O colchete de Poisson em $N,\{\cdot, \cdot\}^{\prime}$, de acordo com (5.35), é dado por:

$$
\{H, G\}^{\prime}=\left[\{\widetilde{H}, \widetilde{G}\}+\sum_{i, j=1}^{2}\left\{\Phi_{i}, \widetilde{H}\right\} a_{i j}\left\{\Phi_{j}, \widetilde{G}\right\}\right] .
$$


Mas

$$
\left\{\Phi_{x}, \Phi_{x}\right\}=\left\{\Phi_{y}, \Phi_{y}\right\}=0
$$

e

$$
\left\{\Phi_{x}, \Phi_{y}\right\}=-\left\{\Phi_{y}, \Phi_{x}\right\}=-\rho \Gamma_{1}
$$

donde

$$
\mathbf{A}=\left(\begin{array}{cc}
0 & \frac{1}{\rho \Gamma_{1}} \\
-\frac{1}{\rho \Gamma_{1}} & 0
\end{array}\right)
$$

Por outro lado, temos que, para toda $F \in C^{\infty}(M)$ :

$$
\begin{gathered}
\left\{\Phi_{x}, F\right\}=\sum_{j=1}^{N} \sum_{i=1}^{2}\left(\frac{\partial \Phi_{x}}{\partial x_{j_{i}}} \frac{\partial F}{\partial p_{j_{i}}}-\frac{\partial \Phi_{x}}{\partial p_{j_{i}}} \frac{\partial F}{\partial x_{j_{i}}}\right)=\frac{\partial \Phi_{x}}{\partial y_{1}} \frac{\partial F}{\partial p_{y_{1}}}-\frac{\partial \Phi_{x}}{\partial p_{x_{1}}} \frac{\partial F}{\partial x_{1}}= \\
=-\frac{\rho \Gamma_{1}}{2} \frac{\partial F}{\partial p_{y_{1}}}-\frac{\partial F}{\partial x_{1}}, \\
\left\{\Phi_{y}, F\right\}=\sum_{j=1}^{N} \sum_{i=1}^{2}\left(\frac{\partial \Phi_{y}}{\partial x_{j_{i}}} \frac{\partial F}{\partial p_{j_{i}}}-\frac{\partial \Phi_{y}}{\partial p_{j_{i}}} \frac{\partial F}{\partial x_{j_{i}}}\right)=\frac{\partial \Phi_{y}}{\partial x_{1}} \frac{\partial F}{\partial p_{x_{1}}}-\frac{\partial \Phi_{x}}{\partial p_{y_{1}}} \frac{\partial F}{\partial y_{1}}= \\
=\frac{\rho \Gamma_{1}}{2} \frac{\partial F}{\partial p_{x_{1}}}-\frac{\partial F}{\partial y_{1}} .
\end{gathered}
$$

Se definimos, para toda $F \in C^{\infty}(M)$ :

$$
\overrightarrow{\mathrm{W}}_{\mathbf{F}}=\left(\begin{array}{c}
\left\{\Phi_{x}, F\right\} \\
\left\{\Phi_{y}, F\right\}
\end{array}\right)=\left(\begin{array}{c}
-\frac{\rho \Gamma_{1}}{2} \frac{\partial \widetilde{H}}{\partial p_{y_{1}}}-\frac{\partial \widetilde{H}}{\partial x_{1}} \\
-\frac{\partial \widetilde{H}}{\partial y_{1}}+\frac{\rho \Gamma_{1}}{2} \frac{\partial \widetilde{H}}{\partial p_{x_{1}}}
\end{array}\right),
$$

precisamos, para conhecer o colchete de Poisson em $N$, calcular $\overrightarrow{\mathrm{w}}_{\widetilde{\mathbf{H}}}^{\mathrm{T}} A \overrightarrow{\mathrm{w}}_{\widetilde{\mathrm{G}}}$ :

$$
\begin{aligned}
\overrightarrow{\mathrm{w}}_{\widetilde{\mathbf{H}}}^{\mathrm{T}} A \overrightarrow{\mathrm{w}}_{\widetilde{\mathrm{G}}}= & \frac{1}{\rho \Gamma_{1}}\left(\frac{\partial \widetilde{G}}{\partial x_{1}} \frac{\partial \widetilde{G}}{\partial y_{1}}-\frac{\partial \widetilde{H}}{\partial y_{1}} \frac{\partial \widetilde{G}}{\partial x_{1}}\right)+\frac{\rho \Gamma_{1}}{4}\left(\frac{\partial \widetilde{H}}{\partial p_{x_{1}}} \frac{\partial \widetilde{G}}{\partial p_{y_{1}}}-\frac{\partial \widetilde{H}}{\partial p_{y_{1}}} \frac{\partial \widetilde{G}}{\partial p_{x_{1}}}\right) \\
& -\frac{1}{2}\left(\frac{\partial \widetilde{H}}{\partial x_{1}} \frac{\partial \widetilde{G}}{\partial p_{x_{1}}}+\frac{\partial \widetilde{H}}{\partial y_{1}} \frac{\partial \widetilde{G}}{\partial p_{y_{1}}}-\frac{\partial \widetilde{H}}{\partial p_{y_{1}}} \frac{\partial \widetilde{G}}{\partial x_{1}}-\frac{\partial \widetilde{H}}{\partial p_{y_{1}}} \frac{\partial \widetilde{G}}{\partial y_{1}}\right)
\end{aligned}
$$

e de

$$
\begin{aligned}
\{\widetilde{H}, \widetilde{G}\}= & \frac{\partial \widetilde{H}}{\partial x_{1}} \frac{\partial \widetilde{G}}{\partial p_{x_{1}}}+\frac{\partial \widetilde{H}}{\partial y_{1}} \frac{\partial \widetilde{G}}{\partial p_{y_{1}}}-\frac{\partial \widetilde{H}}{\partial p_{x_{1}}} \frac{\partial \widetilde{G}}{\partial x_{1}}-\frac{\partial \widetilde{H}}{\partial p_{y_{1}}} \frac{\partial \widetilde{G}}{\partial y_{1}} \\
& +\sum_{j=2}^{N} \sum_{i=1}^{2}\left(\frac{\partial \widetilde{H}}{\partial x_{j_{i}}} \frac{\partial \widetilde{G}}{\partial p_{j_{i}}}-\frac{\partial \widetilde{H}}{\partial p_{j_{i}}} \frac{\partial \widetilde{G}}{\partial x_{j_{i}}}\right)
\end{aligned}
$$


a expressão (5.38) fica:

$$
\begin{aligned}
\{H, G\}^{\prime}= & {\left[\{\widetilde{H}, \widetilde{G}\}+w_{H}^{\top} C z_{G}\right]_{N}=} \\
= & {\left[\frac{1}{\rho \Gamma_{1}}\left(\frac{\partial \widetilde{H}}{\partial x_{1}} \frac{\partial \widetilde{G}}{\partial y_{1}}-\frac{\partial \widetilde{H}}{\partial y_{1}} \frac{\partial \widetilde{G}}{\partial x_{1}}\right)+\frac{\rho \Gamma_{1}}{4}\left(\frac{\partial \widetilde{H}}{\partial p_{x_{1}}} \frac{\partial \widetilde{G}}{\partial p_{y_{1}}}-\frac{\partial \widetilde{H}}{\partial p_{y_{1}}} \frac{\partial \widetilde{G}}{\partial p_{x_{1}}}\right)\right.} \\
& +\frac{1}{2}\left(\frac{\partial \widetilde{H}}{\partial x_{1}} \frac{\partial \widetilde{G}}{\partial p_{x_{1}}}+\frac{\partial \widetilde{H}}{\partial y_{1}} \frac{\partial \widetilde{G}}{\partial p_{y_{1}}}-\frac{\partial \widetilde{H}}{\partial p_{x_{1}}} \frac{\partial \widetilde{G}}{\partial x_{1}}-\frac{\partial \widetilde{H}}{\partial p_{y_{1}}} \frac{\partial \widetilde{G}}{\partial y_{1}}\right) \\
& \left.\left.+\sum_{j=2}^{N} \sum_{i=1}^{2}\left(\frac{\partial \widetilde{H}}{\partial x_{j_{i}}} \frac{\partial \widetilde{G}}{\partial p_{j_{i}}}-\frac{\partial \widetilde{H}}{\partial p_{j_{i}}} \frac{\partial \widetilde{G}}{\partial x_{j_{i}}}\right)\right)\right]_{N}= \\
= & \frac{1}{\rho \Gamma_{1}}\left[\left(\frac{\partial \widetilde{H}}{\partial x_{1}}-\frac{\rho \Gamma_{1}}{2} \frac{\partial \widetilde{H}}{\partial p_{y_{1}}}\right)\left(\frac{\partial \widetilde{G}}{\partial y_{1}}-\frac{\rho \Gamma_{1}}{2} \frac{\partial \widetilde{G}}{\partial p_{x_{1}}}\right)\right. \\
& \left.-\left(\frac{\partial \widetilde{H}}{\partial y_{1}}+\frac{\rho \Gamma_{1}}{2} \frac{\partial \widetilde{H}}{\partial p_{x_{1}}}\right)\left(\frac{\partial \widetilde{G}}{\partial x_{1}}-\frac{\rho \Gamma_{1}}{2} \frac{\partial \widetilde{G}}{\partial p_{y_{1}}}\right)\right]_{N} \\
& +\sum_{j=2}^{N} \sum_{i=1}^{2}\left(\frac{\partial \widetilde{H}}{\partial x_{j_{i}}} \frac{\partial \widetilde{G}}{\partial p_{j_{i}}}-\frac{\partial \widetilde{H}}{\partial p_{j_{i}}} \frac{\partial \widetilde{G}}{\partial x_{j_{i}}}\right) .
\end{aligned}
$$

Se notamos que:

$$
H\left(\vec{x}_{1}, \vec{x}_{2}, \cdots, \vec{x}_{n}, \vec{p}_{x_{2}}, \cdots, \vec{p}_{x_{n}}\right)=\widetilde{H}\left(\vec{x}_{1}, \vec{x}_{2}, \cdots, \vec{x}_{n}, p_{x_{1}}=\frac{\rho \Gamma_{1}}{2} y_{1}, p_{y_{1}}=-\frac{\rho \Gamma_{1}}{2} x_{1} \ldots, \vec{p}_{x_{n}}\right)
$$

onde $\vec{x}_{i}=\left(x_{i}, y_{i}\right)=\left(x_{i_{1}}, x_{i_{2}}\right), \vec{p}_{\vec{x}_{i}}=\left(p_{x_{i}}, p_{y_{i}}\right)=\left(p_{i_{1}}, p_{i_{2}}\right), i=2, \ldots, n$, temos:

$$
\frac{\partial H}{\partial x_{1}}=\left[\frac{\partial \widetilde{H}}{\partial x_{1}}-\frac{\rho \Gamma_{1}}{2} \frac{\partial \widetilde{H}}{\partial p_{y_{1}}}\right]_{N} \quad \frac{\partial H}{\partial y_{1}}=\left[\frac{\partial \widetilde{H}}{\partial y_{1}}-\frac{\rho \Gamma_{1}}{2} \frac{\partial \widetilde{H}}{\partial p_{x_{1}}}\right]_{N},
$$

com expressões análogas para a derivada de $G$ em relação a $x_{1}$ e $y_{1}$. Substituindo a expressão (5.41) diretamente em (5.40), obtemos:

$$
\{H, G\}^{\prime}=\frac{1}{\rho \Gamma_{1}}\left(\frac{\partial H}{\partial x_{1}} \frac{\partial G}{\partial y_{1}}-\frac{\partial H}{\partial y_{1}} \frac{\partial G}{\partial x_{1}}\right)+\sum_{j=2}^{N} \sum_{i=1}^{2}\left(\frac{\partial \widetilde{H}}{\partial x_{j_{i}}} \frac{\partial \widetilde{G}}{\partial p_{j_{i}}}-\frac{\partial \widetilde{H}}{\partial p_{j_{i}}} \frac{\partial \widetilde{G}}{\partial x_{j_{i}}}\right) .
$$

Deste resultado é imediato que a forma simplética na variedade $N$ tem a forma:

$$
w=\rho \Gamma_{1} d y_{1} \wedge d x_{1}+\sum_{i=2}^{N} d p_{x_{i}} \wedge d x_{i}+d p_{y_{i}} \wedge d y_{i}
$$

e a Hamiltoniana é dada por

$$
H=\sum_{i=2}^{N} \frac{1}{2 m_{i}}\left\|p_{i}-\frac{\rho \Gamma_{i}}{2}\left(x_{i} \times e_{3}\right)\right\|^{2}+\rho W .
$$


Portanto, demonstramos o seguinte resultado:

Teorema 5.3.1 O sistema misto de um vórtice sem massa e $N-1$ vórtices com massa, com função Hamiltoniana $H$ e forma simplética $w$ definidas em (5.43) e (5.44) respectivamente, é obtido a partir do sistema de $N$ vórtices com massa $\left(\mathbb{R}^{4 N}, w, H\right)$, com função Hamiltoniana e forma simplética definidas em (4.5) e (4.6), respectivamente, quando restrito à variedade $N$, definida em (5.36).

Observação 5.6 Este processo pode ser iterado, de modo a obter o sistema Hamiltoniano correspondente a $m$ vórtices sem massa e $N-m$ vórtices com massa, ou mesmo o modelo dos vórtices sem massa, quando todas as massas são nulas, definido por (3.14) e (3.15). Deste modo, obtemos o sistema Hamiltoniano $(N, w, H)$, onde $N$ é isomorfa a $\mathbb{R}^{(4 N-2 m)}$, com função Hamiltoniana dada por

$$
H=\sum_{j=m+1}^{N} \frac{1}{2 m_{j}}\left\|p_{j}-\frac{\rho \Gamma_{j}}{2}\left(x_{j} \times e_{3}\right)\right\|^{2}+\rho W
$$

e forma simplética

$$
w=\sum_{j=1}^{m} \rho \Gamma_{1} d y_{1} \wedge d x_{1}+\sum_{i=m+1}^{N} d p_{x_{i}} \wedge d x_{i}+d p_{y_{i}} \wedge d y_{i}
$$

Uma questão que se levanta é a de saber qual a relação entre as integrais primeiras do modelo dos vórtices com massa e as integrais primeiras do modelo misto dos vórtices. O seguinte teorema responde a esta questão.

Teorema 5.3.2 Seja $\Pi$ uma integral primeira do sistema dos vórtices com massa, definido por (5.43) e (5.44). Então $\left.\Pi\right|_{N}$ é uma integral primeira do sistema misto dos vórtices, definido por (5.45) e (5.46) se e somente se

$$
\left.\overrightarrow{\mathrm{w}}_{\Pi}^{\top} A \overrightarrow{\mathrm{w}}_{\mathbf{H}}\right|_{N}=0,
$$

com $\overrightarrow{\mathrm{w}}$ definidos em (5.39). 
Demonstração. Se П é uma integral primeira do sistema dos vórtices com massa, tem-se $\{\Pi, \widetilde{H}\}=0$. Portanto, $\left.\Pi\right|_{N}$ será integral primeira do modelo misto dos vórtices, se e somente se,

$$
\left\{\left.\Pi\right|_{N}, H\right\}^{\prime}=\{\Pi, \widetilde{H}\}-\overrightarrow{\mathbf{w}}_{\Pi}^{\top} A \overrightarrow{\mathbf{w}}_{\mathbf{H}}=0
$$

Como $\{\Pi, \tilde{H}\}=0$, a tese seque imediatamente.

Do teorema acima podemos concluir o sequinte corolário:

Corolario 5.3.1 As restrições das integrais primeiras $\Pi_{x}, \Pi_{y}$ e $J$, do modelo dos vórtices com massa, à variedade $N$, são integrais primeiras do modelo misto dos vórtices, com o primeiro vórtice sem massa.

Demonstração Basta observar que

$$
\left.\overrightarrow{\mathrm{w}}_{\Pi_{\mathbf{x}}}\right|_{N}=\left.\overrightarrow{\mathrm{w}}_{\boldsymbol{\Pi}_{\mathbf{y}}}\right|_{N}=\left.\overrightarrow{\mathrm{w}}_{\mathbf{J}}\right|_{N}=0
$$

Então, temos que:

$$
\begin{aligned}
\bar{\Pi}_{x}=\left.\Pi_{x}\right|_{N} & =\rho \Gamma_{1} y_{1}+\sum_{i=2}^{N} p_{x_{i}}+\frac{\rho \Gamma_{i} y_{i}}{2} \\
\bar{\Pi}_{y}=\Pi_{y} \mid N & =-\rho \Gamma_{1} x_{+} \sum_{i=2}^{N} p_{y_{i}}-\frac{\rho \Gamma_{i} x_{i}}{2} \\
\bar{J}=\left.J\right|_{N} & =-\frac{\rho \Gamma_{i}}{2}\left(x_{1}^{2}+y_{1}^{2}\right)+\sum_{i=2}^{N} x_{i} p_{y_{i}}-y_{i} p_{x_{i}}
\end{aligned}
$$

são integrais primeiras do modelo com um vórtice sem massa. Calculando o colchete de Poisson entre as diversas integrais primeiras, tem-se:

$$
\begin{aligned}
\left\{\bar{\Pi}_{x}, \bar{\Pi}_{y}\right\}^{\prime} & =-\rho \sum_{i=1}^{N} \Gamma_{i}, \\
\left\{\bar{\Pi}_{x}, \bar{J}\right\}^{\prime} & =\bar{\Pi}_{y}, \\
\left\{\bar{\Pi}_{y}, \bar{J}\right\}^{\prime} & =-\bar{\Pi}_{x} .
\end{aligned}
$$


Observação 5.7 Notemos que as integrais primeiras $\bar{\Pi}_{x}, \bar{\Pi}_{y}$ e $\bar{J}$ não estão em involução, mas definindo

$$
\bar{\Pi}^{2}=\bar{\Pi}_{x}^{2}+\bar{\Pi}_{y}^{2}
$$

verifica-se que

$$
\left\{\bar{\Pi}^{2}, \bar{J}\right\}^{\prime}=0 .
$$

Da observação anterior temos a seguinte proposição:

Proposição 5.3.3 O sistema Hamiltoniano, definido por (5.43) e (5.44), tem como integrais primeiras independentes em involução $\bar{J}$ e $\bar{\Pi}^{2}$, além da energia $H$.

A proposição acima e o teorema de Liouville garantem a possibilidade de reduzir o sistema Hamiltoniano, definido por (5.43) e (5.44), em três graus de liberdade. Em particular, o sistema de um vórtice com massa e outro vórtice sem massa é sempre integrável.

\subsection{Exemplo 6: dois vórtices, um com massa e outro sem}

O movimento de dois vórtices, um com massa e outro sem, é determinado pelo sistema hamiltoniano $\left(\mathbb{R}^{6}, \mathrm{w}, \mathrm{H}\right)$, com função Hamiltoniana $H$, definida em (5.45), dada por:

$$
H\left(\vec{x}_{1}, \vec{x}_{2}, \vec{p}_{x_{2}}\right)=\frac{1}{2 m}\left\|\vec{p}_{\vec{x}_{2}}-\frac{\rho \Gamma_{2}}{2}\left(\vec{x}_{2} \times \vec{e}_{3}\right)\right\|^{2}-\frac{\rho \Gamma_{1} \Gamma_{2}}{2 \pi} \ln \left\|\vec{x}_{2}-\vec{x}_{1}\right\|
$$

onde $\vec{x}_{i}=\left(x_{i}, y_{i}\right), i=1,2$ e $\vec{p}_{\vec{x}_{2}}=\left(p_{x_{2}}, p_{y_{2}}\right)$, e com forma simplética $w$ :

$$
w=\rho \Gamma_{1} d y_{1} \wedge d x_{1}+d p_{x_{2}} \wedge d x_{2}+d p_{y_{2}} \wedge y_{2}
$$

Primeiro caso: $\Gamma_{1}+\Gamma_{2}=0$ 
De acordo com (5.49), temos que as integrais primeiras do sistema são:

$$
\begin{aligned}
\Pi_{x} & =\rho \Gamma_{1} y_{1}+\frac{\rho \Gamma_{2}}{2} y_{2}+p_{x_{2}}, \\
\Pi_{y} & =-\rho \Gamma_{1} x_{1}-\frac{\rho \Gamma_{2}}{2} x_{2}+p_{y_{2}}, \\
J & =-\frac{\rho \Gamma_{1}}{2}\left(x_{1}^{2}+y_{1}^{2}\right)+x_{2} p_{y_{2}}-y_{2} p_{x_{2}} .
\end{aligned}
$$

Guiados pela simetria de rotação da função Hamiltoniana (5.53), faremos a seguinte mudança de coordenadas

$$
\begin{aligned}
& \vec{x}_{1}=T_{\theta} \vec{x}, \\
& \vec{x}_{2}=r \cos \theta \vec{e}_{1}+r \sin \theta \vec{e}_{2},
\end{aligned}
$$

onde $T_{\theta}$ é definido em $(4.20)$ e $\vec{x}=(x, y)$.

Os novos momentos $p_{r}$ e $p_{\theta}$ são definidos pelas relações:

$$
\begin{aligned}
& p_{r}=p_{x_{2}} \cos \theta+p_{y_{2}} \sin \theta, \\
& p_{\theta}=-r p_{x_{2}} \sin \theta+r p_{y_{2}} \cos \theta,
\end{aligned}
$$

de onde obtemos

$$
\vec{p}_{\vec{x}_{2}}=T_{\theta} M_{r}^{-1} \vec{p}_{\vec{r}_{2}}
$$

com $M_{r}$ definido em $(4.20)$ e $\vec{p}_{\vec{r}_{2}}=\left(\vec{p}_{r}, \vec{p}_{\theta}\right)$.

A função Hamiltoniana (5.53), nas novas coordenadas, é dada por:

$$
H=\frac{1}{2 m}\left[p_{r}^{2}+\left(\frac{p_{\theta}}{r}+\frac{\rho \Gamma_{2}}{2} r\right)^{2}\right]-\frac{\rho \Gamma_{1} \Gamma_{2}}{4 \pi} \ln \left[(r-x)^{2}+y^{2}\right]
$$

e a forma simplética (5.54):

$$
w=\rho \Gamma_{1}(d y \wedge d x-x d x \wedge d \theta-y d y \wedge d \theta)+d p_{r} \wedge d r+d p_{\theta} \wedge d \theta
$$

Notemos que, de acordo com (5.55), a integral primeira $J$, nas novas variáveis, é dada por:

$$
J=p_{\theta}-\frac{\rho \Gamma_{1}}{2}\left(x^{2}+y^{2}\right)
$$


Motivados por (5.61), definamos:

$$
\begin{aligned}
& \tilde{p}_{\theta}=p_{\theta}-\frac{\rho \Gamma_{1}}{2}\left(x^{2}+y^{2}\right), \\
& p_{x}=\rho \Gamma_{1} y
\end{aligned}
$$

de onde obtemos as integrais primeiras $J$ e $\Pi$ na forma

$$
J=\tilde{p}_{\theta}
$$

$\mathrm{e}$

$$
\left[\begin{array}{l}
\Pi_{x} \\
\Pi_{y}
\end{array}\right]=T_{-\theta}\left[\begin{array}{c}
p_{x}+p_{r} \\
-\rho \Gamma_{1} x-\frac{\rho \Gamma_{2}}{2} r+\frac{\tilde{p}_{\theta}+\frac{\rho \Gamma_{1}}{2}\left(x^{2}+\left(\frac{p_{x}}{\rho \Gamma_{1}}\right)^{2}\right)}{r}
\end{array}\right],
$$

onde $T_{\theta}$ é definido em (4.20).

Note que nas variáveis $\left(x, p_{x}, \theta, \tilde{p}_{\theta}, r, p_{r}\right)$ a forma simplética é:

$$
w=d p_{x} \wedge d x+d p_{r} \wedge d r+d \tilde{p}_{\theta} \wedge d \theta
$$

ou seja, a mudança para estas variáveis é uma transformação canônica.

Dado que $\tilde{p}_{\theta}$ é integral primeira, o sistema é reduzido a dois graus de libertade (4 variáveis). Assim, conhecida a solução do sistema $(\widetilde{H}, \tilde{w})$, com função Hamiltoniana

$$
\widetilde{H}\left(r, p_{r}, x, y\right)=H\left(r, p_{r}, x, y ; \tilde{p}_{\theta}\right)
$$

e forma simplética:

$$
\tilde{w}=d p_{x} \wedge d x+d p_{r} \wedge d r
$$

podemos determinar o valor de $\theta$ através da integração:

$$
\theta=\theta(0)+\int_{0}^{t} \frac{\partial H}{\partial \tilde{p}_{\theta}} d t .
$$

Utilizando a nova transformação canônica:

$$
\begin{array}{ll}
r=w+z, & p_{r}=\frac{p_{w}+p_{z}}{2}, \\
x=w-z, & p_{x}=\frac{p_{w}-p_{z}}{2},
\end{array}
$$


a Hamiltoniana (5.65) fica:

$$
\begin{gathered}
\widetilde{H}=\frac{1}{2 m}\left[\left(\frac{p_{w}+p_{z}}{2}\right)^{2}+\left[\frac{\tilde{p}_{\theta}+\frac{\rho \Gamma_{1}}{2}\left((w-z)^{2}+\left(\frac{p_{w}-p_{z}}{2 \rho \Gamma_{1}}\right)^{2}\right)}{w+z}+\frac{\rho \Gamma_{2}}{2}(w+z)\right]^{2}\right]- \\
-\frac{\rho \Gamma_{1} \Gamma_{2}}{4 \pi} \ln \left[(2 z)^{2}+\left(\frac{p_{w}-p_{z}}{2 \rho \Gamma_{1}}\right)^{2}\right]
\end{gathered}
$$

e a forma simplética (5.66):

$$
\tilde{w}=d p_{z} \wedge d z+d p_{w} \wedge d w
$$

Para reduzir ainda mais o número de graus de libertade na Hamiltoniana (5.65), utilizaremos a integral primeira $\Pi^{2}$. Como a partir de qualquer condição inicial podemos fazer $\Pi=0$ através de uma translação conveniente, desde que $\Gamma_{1}+\Gamma_{2} \neq 0$, suporemos, sem perda de generalidade, que $\Pi^{2}=0$. Escrevendo (5.64) em termos das novas variáveis (5.67), obtemos:

$$
p_{w}=0
$$

donde

$$
\dot{p}_{w}=0
$$

e

$$
4 \rho^{2} \Gamma_{1}\left(\Gamma_{1}+\Gamma_{2}\right)(w+z)-\left(p_{z}^{2} 8 \rho \Gamma_{1} \tilde{p}_{\theta}+16 \rho^{2} \Gamma_{1}^{2} z^{2}\right)=0
$$

donde

$$
w=-z+\frac{\sqrt{p_{z}^{2}+8 \rho \Gamma_{1} \tilde{p}_{\theta}+16 \rho^{2} \Gamma_{1}^{2} z^{2}}}{2 \rho \sqrt{\Gamma_{1}\left(\Gamma_{1}+\Gamma_{2}\right)}} .
$$

Utilizando as relações (5.70) e (5.71) diretamente na Hamiltoniana (5.68), eliminamos as variáveis $p_{w}$ e $w$, obtendo assim o sistema $\left(H^{\prime}, w^{\prime}\right)$, que é também Hamiltoniano, com função Hamiltoniana $H^{\prime}$ dada por:

$$
H^{\prime}\left(z, p_{z}\right)=\widetilde{H}\left(z, p_{z}, w=w\left(z, p_{z} ; p_{w}, \tilde{p}_{\theta}\right), p_{w}=0\right)
$$

com $w=w\left(z, p_{z} ; p_{w}, \tilde{p}_{\theta}\right)$ definido em (5.71) e forma simplética

$$
w^{\prime}=d p_{z} \wedge d z
$$


De fato, é facil ver que as equações que determinam o sistema Hamiltoniano $\left(H^{\prime}, w^{\prime}\right)$ são equivalentes ao sistema anterior, isto é:

$$
\begin{aligned}
\dot{z} & =\frac{\partial H^{\prime}}{\partial p_{z}}=\frac{\partial \widetilde{H}}{\partial p_{z}}+\frac{\partial \widetilde{H}}{\partial w} \frac{\partial w}{\partial p_{z}}=\frac{\partial \widetilde{H}}{\partial p_{z}} \\
\dot{p}_{z} & =-\frac{\partial H^{\prime}}{\partial z}=-\frac{\partial \widetilde{H}}{\partial z}-\frac{\partial \widetilde{H}}{\partial w} \frac{\partial w}{\partial z}=-\frac{\partial \widetilde{H}}{\partial z}
\end{aligned}
$$

onde utilizou-se (5.70):

$$
\dot{p_{w}}=-\frac{\partial H^{\prime}}{\partial w}=0
$$

A Hamiltoniana reduzida é:

$$
\begin{aligned}
H^{\prime}=\frac{1}{2 m}\left[\frac { p _ { z } ^ { 2 } } { 4 } \left[\frac{\tilde{p}_{\theta}+\frac{\rho \Gamma_{1}}{2}\left[\left(-2 z+\frac{1}{2 \rho} \sqrt{\frac{p_{z}^{2}+8 \rho \Gamma_{1} \tilde{p}_{\theta}+16 \rho^{2} \Gamma_{1}^{2} z^{2}}{\Gamma_{1}\left(\Gamma_{1}+\Gamma_{2}\right)}}\right)^{2}+\frac{p_{z}^{2}}{4 \rho^{2} \Gamma_{1}^{2}}\right]}{\frac{1}{2 \rho} \sqrt{\frac{p_{z}^{2}+8 \rho \Gamma_{1} \tilde{p}_{\theta}+16 \rho^{2} \Gamma_{1}^{2} z^{2}}{\Gamma_{1}\left(\Gamma_{1}+\Gamma_{2}\right)}}}+\right.\right. \\
\left.\left.+\frac{\Gamma_{2}}{4} \sqrt{\frac{p_{z}^{2}+8 \rho \Gamma_{1} \tilde{p}_{\theta}+16 \rho^{2} \Gamma_{1}^{2} z^{2}}{\Gamma_{1}\left(\Gamma_{1}+\Gamma_{2}\right)}}\right]^{2}\right]-\frac{\rho \Gamma_{1} \Gamma_{2}}{4 \pi} \ln \left[4 z^{2}+\frac{p_{z}^{2}}{4 \rho^{2} \Gamma_{1}^{2}}\right]
\end{aligned}
$$

Fazendo a mudança de coordenadas

$$
z=\frac{R}{4 \Gamma_{1}} \cos (\phi), \quad p_{z}=R \sin (\phi),
$$

a Hamiltoniana (5.75) fica

$$
H^{\prime}=\frac{\frac{2 \Gamma_{1}+\Gamma_{2}}{4 \Gamma_{1}} R^{2}-\frac{1}{2} \sqrt{\frac{\Gamma_{1}+\Gamma_{2}}{\Gamma_{1}}\left(R^{2}+8 \Gamma_{1} \tilde{p}_{\theta}\right)} R \cos (\phi)+2\left(\Gamma_{1}+\Gamma_{2}\right) \tilde{p}_{\theta}}{2 m}-\frac{R^{2}}{4 \Gamma_{1}}
$$

e a forma simplética (5.73)

$$
w^{\prime}=\frac{R}{4 \Gamma_{1}} d R \wedge d \phi
$$

Agora, definindo

$$
I=\frac{R^{2}}{8 \Gamma_{1}}
$$

podemos enunciar a seguinte proposição: 
Proposição 5.4.1 O sistema Hamiltoniano $(H, w)$, com $H$ e w definidos em (5.53) e (5.54) respectivamente, desde que $\Gamma_{1}+\Gamma_{2} \neq 0$, pode ser reduzido ao sistema Hamiltoniano $\left(H^{\prime}, w^{\prime}\right)$ com um grau de liberdade, dependendo da integral primeira $\tilde{p}_{\theta}$ como parâmetro, com $H^{\prime}$ definida por

$$
H^{\prime}=\frac{\left(2 \Gamma_{1}+\Gamma_{2}\right) I-2 \sqrt{\Gamma_{1}\left(\Gamma_{1}+\Gamma_{2}\right) I\left(I+\tilde{p}_{\theta}\right)} \cos (\phi)+\Gamma_{1}+\Gamma_{2} \tilde{p}_{\theta}}{m}-\frac{\Gamma_{1} \Gamma_{2}}{4 \pi} \ln (I)
$$

e com forma simplética $w^{\prime}$

$$
w^{\prime}=d I \wedge d \phi
$$

Embora não seja possível achar explicitamente a solução do sistema Hamiltoniano acima, é possível investigar qualitativamente seu retrato de fase, seja através de estudos numéricos seja pelo estudo analítico de suas propriedades. Em particular, foi mostrado em [12], que todas as órbitas são limitadas.

Segundo caso: $\Gamma_{1}+\Gamma_{2}=0$

Considerando os valores das intensidades $\Gamma_{2}=-\Gamma_{1}=\Gamma$, temos que a Hamiltoniana (5.53) escreve-se

$$
H\left(\vec{x}_{1}, \vec{x}_{2}, \vec{p}_{x_{2}}\right)=\frac{1}{2 m}\left\|\vec{p}_{\overrightarrow{x_{2}}}-\frac{\rho \Gamma}{2}\left(\vec{x}_{2} \times \vec{e}_{3}\right)\right\|^{2}+\frac{\rho \Gamma^{2}}{2 \pi} \ln \left\|\vec{x}_{2}-\vec{x}_{1}\right\|
$$

e a forma simplética (5.54)

$$
w=-\rho \Gamma d y_{1} \wedge d x_{1}+d p_{x_{2}} \wedge d x_{2}+d p_{y_{2}} \wedge d y_{2}
$$

De acordo com (5.55), este sistema tem as integrais primeiras:

$$
\begin{aligned}
\Pi_{x} & =-\rho \Gamma y_{1}+\frac{\rho \Gamma}{2} y_{2}+p_{x_{2}}, \\
\Pi_{y} & =\rho \Gamma x_{1}-\frac{\rho \Gamma_{2}}{2} x_{2}+p_{y_{2}}, \\
J & =\frac{\rho \Gamma}{2}\left(x_{1}^{2}+y_{1}^{2}\right)+x_{2} p_{y_{2}}-y_{2} p_{x_{2}} .
\end{aligned}
$$

Neste caso, a partir de (5.50), vemos que as integrais primeiras $\Pi_{x}$ e $\Pi_{y}$, estão em involução, isto é, $\left\{\Pi_{x}, \Pi_{y}\right\}^{\prime}=0$ (uma vez que $\Gamma_{1}+\Gamma_{2}=0$ ). Utilizaremos este fato para a 
redução. Consideremos a seguinte translação:

$$
\begin{aligned}
& x_{1}=x_{2}+X, \\
& y_{1}=y_{2}+Y .
\end{aligned}
$$

Nas novas variáveis, as integrais primeiras $\Pi_{x}$ e $\Pi_{y}$, definidas em (5.84), são dadas por:

$$
\begin{aligned}
& \Pi_{x}=-\rho \Gamma Y-\frac{\rho \Gamma}{2} y_{2}+p_{x_{2}} \\
& \Pi_{y}=\rho \Gamma X+\frac{\rho \Gamma}{2} x_{2}+p_{y_{2}} .
\end{aligned}
$$

Motivados por (5.86), definimos:

$$
\begin{aligned}
& \tilde{p}_{x_{2}}=-\rho \Gamma Y-\frac{\rho \Gamma}{2} y_{2}+p_{x_{2}} \\
& \tilde{p}_{y_{2}}=\rho \Gamma X+\frac{\rho \Gamma}{2} x_{2}+p_{y_{2}} .
\end{aligned}
$$

A Hamiltoniana (5.82) nas novas variáveis é dada por:

$$
H=\frac{1}{2 m}\left[\left(\tilde{p}_{x_{2}}+\rho \Gamma Y\right)^{2}+\left(\tilde{p}_{y_{2}}-\rho \Gamma X\right)^{2}\right]+\frac{\rho \Gamma^{2}}{4 \pi} \ln \left[X^{2}+Y^{2}\right]
$$

e a forma simplética (5.83) por

$$
w=\rho \Gamma d X \wedge d Y+d p_{x_{2}} \wedge d x_{2}+d p_{y_{2}} \wedge d y_{2}
$$

É claro que a Hamiltoniana não depende das coordenadas $x_{2}$ e $y_{2}$, já que $\tilde{p}_{x_{2}}$ e $\tilde{p}_{y_{2}}$ são integrais primeiras. Portanto, conhecida a solução do sistema $(\widetilde{H}, \tilde{w})$, com Hamiltoniana $\widetilde{H}$ :

$$
\widetilde{H}(X, Y)=H\left(X, Y ; \tilde{p}_{x_{2}}, \tilde{p}_{y_{2}}\right)
$$

e forma simplética $\tilde{w}$

$$
\tilde{w}=\rho \Gamma d X \wedge d Y
$$


é possível determinar a solução do sistema original através de quadraturas, isto é, se conhecemos as soluções $X(s)$ e $Y(s)$, do sistema $(\tilde{H}, \tilde{w})$, podemos escrever:

$$
\begin{gathered}
x_{1}=x_{2}+X, \\
y_{1}=y_{2}+Y, \\
x_{2}(t)=x_{2}(0)+\frac{\tilde{p}_{x_{2}}}{m} t+\frac{\rho \Gamma}{m} \int_{0}^{t} Y(s) d s, \\
y_{2}(t)=y_{2}(0)+\frac{\tilde{p}_{y_{2}}}{m} t-\frac{\rho \Gamma}{m} \int_{0}^{t} X(s) d s .
\end{gathered}
$$

Escrevendo $X$ e $Y$ em coordenadas polares:

$$
\begin{gathered}
X=R \cos (\phi), \\
Y=R \sin (\phi),
\end{gathered}
$$

a Hamiltoniana (5.90), fica

$$
\widetilde{H}=\frac{1}{2 m}\left[\Gamma^{2} R^{2}+2 \tilde{p}_{x_{2}} \Gamma R \sin (\phi)-2 \tilde{p}_{y_{2}} \Gamma R \cos (\phi)\right]+\frac{\Gamma^{2}}{4 \pi} \ln \left[R^{2}\right]
$$

e a forma simplética (5.91)

$$
\tilde{w}=\rho \Gamma R d R \wedge d \phi
$$

Definindo $A$ e $\theta$ por:

$$
\tilde{p}_{y_{2}}=\frac{A}{2} \cos (\theta), \quad \tilde{p}_{x_{2}}=\frac{A}{2} \sin (\theta)
$$

temos:

$$
\begin{aligned}
A \Gamma R\left(\frac{2 \tilde{p}_{x_{2}}}{A} \sin (\phi)-\frac{2 \tilde{p}_{y_{2}}}{A} \cos (\phi)\right) & =A \Gamma R(\sin (\theta) \sin (\phi)-\cos (\theta) \cos (\phi)) \\
& =A \Gamma R(\cos (\theta+\phi))
\end{aligned}
$$

e, mudando convenientemente a origem da coordenada angular, podemos escrever a $\mathrm{Ha}$ miltoniana (5.95) como:

$$
\widetilde{H}=\frac{\Gamma^{2} R^{2}+A \Gamma R \cos (\phi)}{2 m}+\frac{\Gamma^{2}}{4 \pi} \ln \left[R^{2}\right] .
$$


Fazendo

$$
I=\frac{\Gamma R^{2}}{2}
$$

podemos enunciar a seguinte proposição:

Proposição 5.4.2 O sistema Hamiltoniano $(H, w)$, com $H$ e w definidos em (5.82) e (5.83) respectivamente, quando $\Gamma_{1}+\Gamma_{2}=0$, pode ser reduzido ao sistema Hamiltoniano $(\widetilde{H}, \tilde{w})$ com um grau de liberdade, com $\widetilde{H}$ definida por:

$$
\widetilde{H}=\frac{2 \Gamma I+A \sqrt{2 \Gamma I} \cos (\phi)}{2 m}+\frac{\Gamma^{2}}{4 \pi} \ln [I]
$$

e forma simplética $\tilde{w}$

$$
\tilde{w}=d I \wedge d \phi .
$$

As equações do movimento associado ao sistema $(\widetilde{H}, \tilde{w})$ são

$$
\begin{aligned}
\dot{I} & =\frac{A}{2 m} \sqrt{2 I} \sin (\phi), \\
\dot{\phi} & =\frac{1}{m}+\frac{A}{4 m} \sqrt{\frac{2}{I}} \cos (\phi)+\frac{1}{4 \pi I}
\end{aligned}
$$

onde consideramos $\Gamma=1$, o que é possível fazer redefinindo a variável $I$, a massa e reescalonando o tempo. Para $A \geq 2 \sqrt{\frac{2 m}{\pi}}$, o sistema (5.101) tem os seguintes pontos de equilíbrio:

$$
\begin{aligned}
& \left(I_{1}, \phi_{1}\right)=\left(m \frac{\frac{A^{2}}{8 m}-\frac{1}{2 \pi}-\sqrt{\frac{A^{4}}{64 m^{2}}-\frac{A^{2}}{8 \pi m}}}{2}, \pi\right), \\
& \left(I_{2}, \phi_{2}\right)=\left(m \frac{\frac{A^{2}}{8 m}-\frac{1}{2 \pi}+\sqrt{\frac{A^{4}}{64 m^{2}}-\frac{A^{2}}{8 \pi m}}}{2}, \pi\right) .
\end{aligned}
$$

A matriz M do sistema linearizado de (5.101) é:

$$
\mathbf{M}=\left(\begin{array}{cc}
\frac{A}{4 m} \sqrt{\frac{2}{I}} \sin (\phi) & \frac{A}{2 m} \sqrt{2 I} \cos (\phi) \\
\frac{A}{8 m I} \sqrt{\frac{2}{I}} \cos (\phi) & -\frac{A}{4 m} \sqrt{\frac{2}{I}} \sin (\phi)
\end{array}\right)
$$


Note que o traço de $\mathbf{M}$ é sempre nulo, que $\operatorname{Det}\left(\left.\mathbf{M}\right|_{\left(\mathbf{I}_{1}, \phi_{1}\right)}\right)<0$ e que $\operatorname{Det}\left(\left.\mathbf{M}\right|_{\left(\mathbf{I}_{2}, \phi_{2}\right)}\right)>0$. Portanto, $\left.\mathbf{M}\right|_{\left(\mathbf{I}_{1}, \phi_{1}\right)}$ tem dois autovalores reais de sinais opostos e $\left(I_{1}, \phi_{1}\right)$ será um ponto de sela, enquanto que $\left.\mathbf{M}\right|_{\left(\mathbf{I}_{2}, \phi_{2}\right)}$ tem dois autovalores imaginários puros e $\left(I_{2}, \phi_{2}\right)$ será um centro. Assim, podemos enunciar a seguinte proposição:

Proposição 5.4.3 O sistema de equações diferenciais (5.101), satisfazendo a condição $\frac{A^{2}}{m}>\frac{8}{\pi}$, tem dois pontos de equilibrio

$$
\begin{aligned}
& \left(I_{s}, \phi_{s}\right)=\left(m \frac{\frac{A^{2}}{8 m}-\frac{1}{2 \pi}-\sqrt{\frac{A^{4}}{64 m^{2}}-\frac{A^{2}}{8 \pi m}}}{2}, \pi\right), \\
& \left(I_{c}, \phi_{c}\right)=\left(m \frac{\frac{A^{2}}{8 m}-\frac{1}{2 \pi}+\sqrt{\frac{A^{4}}{64 m^{2}}-\frac{A^{2}}{8 \pi m}}}{2}, \pi\right),
\end{aligned}
$$

sendo $\left(I_{s}, \phi_{s}\right)$ un ponto de sela e $\left(I_{c}, \phi_{c}\right)$ um centro. Além disso, existe uma órbita homoclínica, dada implicitamente pela equação

$$
H\left(I_{s}, \phi_{s}\right)=\frac{2 I+A \sqrt{2 I} \cos (\phi)}{2 m}+\frac{\Gamma^{2}}{4 \pi} \ln [I] .
$$

Se $\frac{A^{2}}{m}=\frac{8}{\pi}$ existe um único ponto de equilibrio $\left(I^{o}, \phi^{o}\right)=\left(m, \frac{1}{4 \pi}\right)$, não hiperbólico, e se $\frac{A^{2}}{m}<\frac{8}{\pi}$ o sistema não tem pontos de equilíbrio.

Note que esta proposição mostra que o parâmetro adequado ao estudo de bifurcações é a energia cinética $T=\frac{A^{2}}{m}$, e não a massa $m$.

Em [12], Gomes considerou o problema misto de três vórtices, num caso restrito: existem dois vórtices sem massa, com intensidades $\Gamma_{1}$ e $\Gamma_{2}$, e um terceiro vórtice com massa $m$ e intensidade $\Gamma_{3}$, de tal forma que $\Gamma_{1}+\Gamma_{2}+\Gamma_{3}=0$ e a intensidade $\Gamma_{1}$ é muito menor que os outros parâmetros. Matematicamente, esta hipótese corresponde a estudar o limite $\Gamma_{1} \rightarrow 0$. Este problema pode ser olhado como uma perturbação do modelo misto, com dois vórtices, descrito nesta seção. Desta forma, foi possível mostrar, analiticamente, que a função de Melnikov (ver seção 3.4) associada a uma certa órbita heteroclínica do problema apresenta zeros simples, e é limitada. Isto mostra que o problema é não 
integrável, pois existem pontos heteroclínicos transversos, e portanto "horseshoes". Por outro lado, em [13], Gomes prova, por meio de uma versão adequada do teorema K.A.M. (ver [3]), que para $m$ suficientemente grande existe um conjunto de condições iniciais deste sistema, com medida de Lebesgue não nula, para as quais o movimento é quasi periódico. Concluímos assim, de forma similar ao que ocorre no sistema de dois vórtices com massa, estudado no capítulo 4, que existem regiões onde as soluções do problema restrito misto dos três vórtices são quasi periódicas e regiões onde apresentam comportamento caótico. 


\section{Bibliografia}

[1] Abraham, R. and Marsden, J., Foudations of Mechanics, Addison-Wesley, 1997.

[2] Abraham, R., Marsden, J. and Ratiu, T., Manifolds Tensor Analysis and Applications, Springer-Verlag, 1998.

[3] Arnold, V., Métodos Matemáticos da Mecânica Clássica, Mir, 1987.

[4] Arnold, V. et al, Dynamical Systems III , Springer-Verlag, 1993.

[5] Castilla, M., Moauro, V., Negrini, P. and Oliva, W., The four positive vortices problem: regions of caotics behavior and non-integrability, Ann. Inst. Henri Poincaré, 59, n.1, 1993.

[6] Chorin, A. J., Vorticity and Turbulence, Springer-Verlag, 1998.

[7] Chorin, A. and Marsden, J., A Mathematical Introducction to Fluid Mechanics, Springer-Verlag, 1979.

[8] Chow, S. and Hale, K., Methods of Bifurcations Theory, Springer-Verlag, 1982.

[9] Driscoll, C. and Fine, K., Experiments on vortex dynamics in pure electron plasma, Phys. Fluids B, vol.2, 1990.

[10] Folland, G., Introduction to Partial Differential Equations, Princeton University Press, 1976.

[11] Friedrichs, K., Special Topics in fluid Dynamics, Nelson, 1966. 
[12] Gomes, D., Dinâmica de Vórtices , Trabalho Final de Curso, Instituto Superior Técnico, Lisboa, Portugal, 1995.

[13] Gomes, D., O Modelo misto dos Vórtices, Dissertação de Mestrado, Instituto Superior Técnico, Lisboa, Portugal, 1998.

[14] Gallavotti, G., The Elements of Mechanics, Springer-Verlag, 1983.

[15] Guckenheimer, J. and Holmes, P., Nolinear Oscilations, Dynamical System, and Bifurcation of Vector Field, Springer-Verlag, 1983.

[16] Gurtin, M., An Introduction to Continuum mechanics, Academic Press, 1981.

[17] Khanin, K.M., Quasi-Periodic Motions of Vortex Systems , Physica 4D Nonlinear Phenomena, 1982.

[18] Koiller, J. and Carvalho, S., Non-Integrability of the 4-Vortex System: Analytical Proof, Commun. Math. Physics 120, 1989.

[19] Marchioro, C. and Pulvirenti, M., Mathematical Theory of Incompressible NonViscous Fluids, preprint, 1994.

[20] Marsden, J.D. and Weinstein, A., Coadjoints orbits, vortices and Clebsch variables for incompressible fluids, Physica 7D, 1983.

[21] Oliva, W., Integrability Problems in Hamiltonian Systems, 1992.

[22] Oliva, W., The Motions of Two Dimensional Vortices with Mass as a Singular Perturbation Hamiltonian Problem, preprint, 1994.

[23] Olver,P., Aplications of Lie Algebras to Differential Equations, Springer-Verlag, 1994.

[24] Ragazzo, C., Nonintegrability of Some Hamiltonian system, Scattering and Analytic Continuation, Commun. Math. Phys. 166, 255-277, 1994. 
[25] Ragazzo, C., Koiller, J. and Oliva, W., On the Motion of two dimensional vortices with Mass, J. of Nonlinear Sciences, Vol.4, 1994.

[26] Sotomayor, J., Lições de Equações Diferenciais Ordinárias, Euclides, 1980.

[27] Synge, J. L., On the Motion of three Vortices, Can. Journal of Math. vol.1, 1949.

[28] Terra, G., Estrutura de Lie-Poisson e aplica̧̧ões em sistema vinculados, Dissertação de Mestrado, IMEUSP, 1999.

[29] Ziglin, S., Non Integrability of a Problem on the Motion of Four Point Vortices, Soviet. Mathematical Dokl.,vol.21, 1980. 